\title{
Supersymmetry algebras in arbitrary signature and their R-symmetry groups
}

\section{Gall and T. Mohaupt}

\author{
Department of Mathematical Sciences, University of Liverpool, \\ Peach Street, Liverpool L69 7ZL, U.K. \\ E-mail: Louis.Gall@astrazeneca.com, Thomas.Mohaupt@liv.ac.uk
}

ABSTRACT: String theory, specifically type-II superstring theory, can be formulated in any ten-dimensional signature. To facilitate the study of supergravity and superstring theories in this setting, we present a uniform construction of supersymmetry algebras in arbitrary dimension and signature, which generalizes the ideas underlying symplectic Majorana spinors. In our formalism R-symmetry acts on an auxiliary multiplicity space which makes its action manifest. This allows us to provide extensive tables which list the R-symmetry groups of extended supersymmetry algebras for all signatures together with other useful information. Twisted ('type-*') supersymmetry algebras in Lorentz signature with non-compact R-symmetry groups are shown to be part of a general pattern resulting from the interplay between complex superbrackets and reality conditions. As an application we show how the relations between type-II string theories in ten and nine dimensions can be extracted from their supersymmetry algebras. We also use our results to determine the special geometry of vector and hypermultiplet scalar manifolds of four-dimensional $\mathcal{N}=2$ and three-dimensional $\mathcal{N}=4$ supergravity theories for all signatures.

KEYwords: String Duality, Superstrings and Heterotic Strings, Supergravity Models, Supersymmetry and Duality

ARXIV EPRINT: 2108.05109 


\section{Contents}

1 Introduction 1

1.1 Background 2

1.2 Overview of the construction 6

1.3 Organisation of the paper 9

2 Clifford and spinor representations $\quad 11$

$\begin{array}{ll}2.1 \text { Clifford and spinor modules } & 11\end{array}$

$\begin{array}{lll}2.2 & \text { Spinor representations in arbitrary signature } & 13\end{array}$

$\begin{array}{lll}2.3 & \text { Complexification of spinor modules } & 16\end{array}$

$\begin{array}{llr}3 & \text { Bilinear forms and complex supersymmetry algebras } & 18\end{array}$

$\begin{array}{lll}3.1 & \text { Bilinear forms on the complex spinor module } \mathbb{S} & 18\end{array}$

$\begin{array}{lll}3.2 & \text { Bilinear forms on the Weyl spinor modules } \mathbb{S}_{ \pm} & 19\end{array}$

3.3 Bilinear forms on the auxiliary space $\mathbb{C}^{K}{ } \quad 20$

3.4 Complex supersymmetry algebras $\quad 21$

$\begin{array}{lll}3.4 .1 & \text { Odd dimensions } & 21\end{array}$

$\begin{array}{ll}3.4 .2 \text { Even dimensions } & 21\end{array}$

3.5 Complex R-symmetry groups 22

3.5.1 Odd dimensions 23

3.5.2 Even dimensions, orthogonal bilinear form 24

3.5.3 Even dimensions, isotropic bilinear forms 25

$\begin{array}{ll}3.5 .4 & \text { Summary table }\end{array}$

4 Reality conditions and $\epsilon$-quaternionic structures $\quad 26$

$4.1 \epsilon$-quaternionic structures on the complex spinor module $\mathbb{S} \quad 26$

$4.2 \epsilon$-quaternionic structures on the auxiliary space $\mathbb{C}^{K} \quad 28$

4.3 Real structures on $\mathbb{S} \otimes \mathbb{C}^{K}$ and $\mathbb{S}_{+} \otimes \mathbb{C}^{K_{+}} \oplus \mathbb{S}_{-} \otimes \mathbb{C}^{K_{-}} \quad 30$

4.3.1 Odd dimensions 30

$\begin{array}{lll}4.3 .2 & \text { Even dimensions } & 30\end{array}$

5 Real supersymmetry algebras $\quad 31$

$\begin{array}{lll}5.1 & \text { Reality conditions and superbrackets } & 32\end{array}$

5.2 Real supersymmetry algebras with $\mathfrak{g}_{1}^{\mathbb{C}}=\mathbb{S} \otimes \mathbb{C}^{K}$

5.3 Real supersymmetry algebras with $\mathfrak{g}_{1}^{\mathbb{C}}=\mathbb{S}_{+} \otimes \mathbb{C}^{K_{+}} \oplus \mathbb{S}_{-} \otimes \mathbb{C}^{K_{-}} \quad 34$

$6 \quad$ R-symmetry groups $\quad \mathbf{3 4}$

6.1 Determination of R-symmetry groups 35

6.1.1 Odd dimensions 35

$\begin{array}{lll}\text { 6.1.2 Orthogonal Weyl-compatible signatures } & 37\end{array}$

$\begin{array}{lll}\text { 6.1.3 Orthogonal Weyl-incompatible signatures } & 37\end{array}$

6.1.4 Isotropic Weyl-compatible signature 38

6.1.5 Isotropic Weyl-incompatible signatures 40 
6.2 Real supersymmetry algebras and their R-symmetry groups 41

6.2.1 $(1,0)$ or $(0,1)$ algebras 41

$6.2 .2(0,2),(1,1),(2,0)$ and $\mathcal{N}=1$ algebras $\quad 42$

6.2.3 General $\left(\mathcal{N}_{+}, \mathcal{N}_{-}\right) \quad 44$

7 Isomorphisms and classification $\quad 44$

$\begin{array}{lll}7.1 & \text { Odd dimensions } & 46\end{array}$

$\begin{array}{lll}7.2 & \text { Orthogonal, Weyl-compatible signatures } & 46\end{array}$

$\begin{array}{lll}7.3 & \text { Orthogonal, Weyl-incompatible signatures } & 47\end{array}$

$\begin{array}{lll}7.4 & \text { Isotropic, Weyl-compatible signatures } & 47\end{array}$

$\begin{array}{lll}7.5 & \text { Isotropic, Weyl-incompatible signatures } & 48\end{array}$

7.6 Remarks on the general classification problem 48

8 Applications to type-II string theories in $D=10,9,4,3 \quad 49$

8.1 $\mathcal{N}=2$ supersymmetry in signature $(1,9)$ and type-II string theories $\quad 49$

$8.2 \mathcal{N}=2$ supersymmetry in general ten-dimensional signatures $\quad 52$

$8.3 \mathcal{N}=2$ supersymmetry in nine dimensions $\quad 53$

8.3.1 Nine-dimensional $\mathcal{N}=2$ supersymmetry algebras $\quad 53$

8.3.2 Reduction of Clifford algebras 54

8.3.3 Reduction of reality conditions $\quad 55$

8.3.4 Reduction of vector-valued bilinear forms 56

8.3.5 Summary of relations between ten-dimensional and nine-dimensional $\mathcal{N}=2$ supersymmetry algebras $\quad 56$

8.4 Four-dimensional $\mathcal{N}=2$ supersymmetry algebras and type-II/II*

$\begin{array}{ll}\text { Calabi-Yau compactifications } & 56\end{array}$

8.5 Three-dimensional $\mathcal{N}=4$ supersymmetry algebras and their hypermultiplet geometries $\quad 58$

9 Conclusion and outlook $\quad 59$

$\begin{array}{ll}\text { A Conventions, notation and some useful formulae } & \mathbf{6 1}\end{array}$

$\begin{array}{lll}\text { A.1 Spinor index conventions } & 61\end{array}$

$\begin{array}{lll}\text { A.2 Index conventions for internal indices } & 62\end{array}$

A.3 Poincaré Lie superalgebras with and without indices 63

A.4 Useful formulae relating to chirality 66

A.5 Proof that a signature flip $(t, s) \leftrightarrow(s, t)$ exchanges $B_{+} \leftrightarrow B_{-} \quad 66$

$\begin{array}{ll}\text { B Real (semi-)spinors and Majorana spinors } & 67\end{array}$

C Details on the complexification of spinor modules 68

$\begin{array}{lr}\text { D Matrix notation for Weyl spinors } & 69\end{array}$

$\begin{array}{ll}\text { D.1 Explanation of the matrix notation } & 69\end{array}$

D.2 Action of R-symmetry transformations on the complex spinor module $\mathbb{S} \quad 71$

$\begin{array}{ll}\text { D.2.1 Orthogonal bilinear forms } & 71\end{array}$

$\begin{array}{lll}\text { D.2.2 Isotropic bilinear forms } & 72\end{array}$ 
$\begin{array}{ll}\text { E Details of isomorphisms } & 73\end{array}$

E.1 The R-transformation $\quad 73$

E.1.1 Orthogonal dimensions 73

E.1.2 Isotropic dimensions $\quad 74$

$\begin{array}{lll}\text { E.2 The S-transformations } & 75\end{array}$

$\begin{array}{lll}\text { E.2.1 } S \text {-transformations and orthogonal bilinear forms } & 75\end{array}$

E.2.2 $S$-transformations and isotropic bilinear forms $\quad 75$

$\begin{array}{lll}\text { E.2.3 } & S_{L} \text { transformations and Weyl-incompatible reality conditions } & 76\end{array}$

E.2.4 $S_{J}$ transformations in the isotropic Weyl-compatible signatures $\quad 77$

E.3 The T-transformation $\quad 77$

E.4 Relations between isotropic Weyl-incompatible supersymmetry algebras $\quad 78$

E.4.1 $S_{J}$-transformation: $(\delta, \delta) \rightarrow(J, J)$ and $(\delta, J) \rightarrow(J, \delta) \quad 79$

E.4.2 F-transformation: $(\delta, J) \rightarrow\left(\delta, I_{k, k}\right) \quad 79$

E.4.3 G-transformation: $\left(J, \tilde{I}_{2 r, 2 s}\right) \rightarrow(J, \delta) \quad 80$

E.4.4 Remaining case $\left(J, I_{1,1}\right) \rightarrow(J, \delta) \quad 80$

$\begin{array}{llr}\text { F Dimensional reduction } & \mathbf{8 0}\end{array}$

F.1 Odd to even dimensions $\quad 81$

F.2 Even to odd dimensions $\quad 81$

\section{Introduction}

String theory extends our concepts of space-time geometry and symmetry in various directions. Besides the well known and widely explored concepts of T-duality, mirror symmetry and the AdS/CFT correspondence, there is the less explored idea of timelike T-duality, which has far reaching consequences. Firstly, in Minkowski signature, it relates the type-II string theories to the so-called type-II* string theories, which realize 'twisted' versions of the standard $\mathcal{N}=2$ supersymmetry algebras [1]. Secondly, together with standard (i.e. spacelike) T-duality and with S-duality, it creates a web of type-II string theories which covers all possible space-time signatures $(t, s), t+s=10$ in ten dimensions [2]. This type-II network is related to three versions of eleven-dimensional M-theory with spacetime signature $(1,10),(2,9),(5,6)$. While the interpretation of theories with multiple time dimensions is not obvious, these exotic theories are arguably part of the configuration space of string theory, and therefore their properties deserve detailed investigation. The effective field theories of type-* theories contain fields with negative kinetic energy, but are, as full string theories, equivalent to standard type-II theories, at least as long as the timelike circle has finite radius. Type-* theories admit de Sitter solutions, and theories with multiple times may admit interesting brane world models. Both formal and phenomenological aspects of exotic string theories, by which we refer to type-* as well as non-Minkowksi signature theories, have been investigated in more detail in [3, 4]. 
Supersymmetry and supergravity in Euclidean and other non-Lorentzian signatures have been studied to some extent in the literature. Supersymmetry algebras in arbitrary signature have been discussed in [5]. Lower-dimensional supergravity theories in nonstandard signatures have been constructed using dimensional reduction in [6-11]. A Euclidean version of the special geometry of $\mathcal{N}=2$ vector and hypermultiplets has been developed in [12-15], while $\mathcal{N}=2$ vector multiplets in arbitrary signature were constructed in $[16,17]$. Four-dimensional supersymmetric solutions in neutral signature have been investigated in $[18,19]$, brane-like solutions in arbitrary dimension and signature have been constructed in [20] and supersymmetric solutions of five-dimensional vector multiplets coupled to supergravity have recently been studied for arbitrary signature in [21].

The concrete form of the supersymmetry algebra varies from dimension to dimension, and from signature to signature, depending on whether the supercharges are Dirac, Majorana, Weyl or Majorana-Weyl spinors. The main result of this paper is a universal construction, which generalizes the idea underlying symplectic Majorana spinors, and applies it to any dimension and signature. The general idea is to start with a complex, hence signature independent supersymmetry algebra and then to impose reality conditions which select a space-time signature and reduce the complex R-symmetry group to one of its real forms. As we will see the possible reality conditions include, besides standard Majorana and symplectic Majorana conditions, the 'twisted' or $\mathrm{O}(p, q)$ Majorana conditions which were used in [2] to describe the supersymmetry algebras of ten-dimensional type-II string theories in general signature. Our formalism provides a systematic way of identifying reality conditions that define real supersymmetry algebras by selecting real forms of the complex R-symmetry group. Such a uniform approach useful if one wants to explore the web of string dualities across dimensions and signatures, as we illustrate using type-II string theories and their compactifications as an example. One advantage of our formalism is that it disentangles the actions of the spin and R-symmetry groups, making the R-symmetry group manifest. This allows one to easily distinguish between non-isomorphic supersymmetry algebras which have the same number of supercharges, and to identify Lorentz signatures where twisted (type-*) supersymmetry algebras exist. For example the ten-dimensional type-IIB and type-IIB* algebras have R-symmetry groups $\mathrm{O}(2)$ and $\mathrm{O}(1,1)$, while the standard and twisted four-dimensional $\mathcal{N}=2$ supersymmetry algebra have R-symmetry groups $\mathrm{U}(2)$ and $\mathrm{U}(1,1)$, respectively. We present tables where we classify the possible R-symmetry groups appearing in our construction up to dimension 12 for all signatures.

\subsection{Background}

Before we give an overview of our construction, we need to provide some background. The following section is partly based on [22], whose method, notation and terminology we have adopted. Given a real vector space $V \cong \mathbb{R}^{t, s}$, equipped with a scalar product (non-degenerate real bilinear form) of signature $(t, s)$, the associated Poincaré Lie algebra is

$$
\mathfrak{g}_{0}=\mathfrak{s o}(V)+V
$$


where $\mathfrak{s o}(V) \cong \mathfrak{s o}(t, s)$ is the 'Lorentz Lie algebra' (Lie algebra of infinitesimal isometries of the scalar product), and where $V$ is the Lie algebra of translations. ${ }^{1}$ To extend this to a Poincaré Lie superalgebra $\mathfrak{g}=\mathfrak{g}_{0}+\mathfrak{g}_{1}$ one adds a real $\operatorname{Spin}(V)$ module $\mathfrak{g}_{1}$, which may be reducible, and introduces a $\mathbb{Z}_{2}$-grading under which $\mathfrak{g}_{0}$ is even, while $\mathfrak{g}_{1}$ is odd,

$$
\mathfrak{g}=\mathfrak{g}_{0}+\mathfrak{g}_{1}=\mathfrak{s o}(V)+V+\mathfrak{g}_{1} .
$$

We will use the terms $\mathfrak{g}$-module, $G$-module, or module for short, for a vector space carrying a representation of a Lie algebra $\mathfrak{g}$ or of a Lie group $G$. The group $\operatorname{Spin}(V)=\operatorname{Spin}(t, s)$, which is a two-fold cover of special orthogonal group $\mathrm{SO}(V)=\mathrm{SO}(t, s)$, is contained in the real Clifford algebra, $\operatorname{Spin}(V) \subset C l(V)=C l_{t, s}$. The same applies to its Lie algebra $\mathfrak{s p i n}(t, s) \cong \mathfrak{s o}(t, s) .^{2}$ The vector space $\mathfrak{g}_{1}$ is a finite sum of irreducible real spinor representations which, depending on signature, can be Dirac spinors, Majorana spinors, Weyl spinors, or Majorana-Weyl spinors. Note that complex representations can be regarded as real representations by 'forgetting the complex structure,' and that it is this underlying real representation that is relevant for the construction and classification of real supersymmetry algebras. In signatures where Majorana spinors do not exist, the elements of the irreducible real spinor representations are Dirac or Weyl spinors.

While the action of $\mathfrak{s o}(V)$ on $\mathfrak{g}_{1}$ is determined by the spinor representations we have chosen, it can be shown that the translation algebra $V$ must operate trivially on $\mathfrak{g}_{1}$, and that the only freedom besides the choice of $\mathfrak{g}_{1}$ is the choice of a superbracket, that is of a symmetric bracket

$$
\Pi: \quad \mathfrak{g}_{1} \times \mathfrak{g}_{1} \rightarrow V, \quad(\lambda, \chi) \mapsto \Pi(\lambda, \chi)=\{\lambda, \chi\},
$$

which is covariant with respect to the action of $\mathfrak{s o}(V)$. Mathematically, such a bracket corresponds to a real, symmetric, non-degenerate, spin-equivariant, vector-valued bilinear form $\Pi$ on the spinor module $\mathfrak{g}_{1}$. In the physics literature the superbracket is usually defined by writing down the anti-commutation relations of the supercharges $Q_{i \alpha}$, where $\alpha$ is a spinor index corresponding to an irreducible representation, while $i=1 \ldots, \mathcal{N}$ labels copies of the irreducible real spinor representation. Let us illustrate this using the case where the irreducible real spinor representation is given by Majorana spinors. Then the $\mathcal{N}$-extended supersymmetry algebra ${ }^{3}$ takes the form

$$
\left\{Q_{i \alpha}, Q_{j \beta}\right\}=M_{i j}\left(\gamma^{\mu} C^{-1}\right)_{\alpha \beta} P_{\mu} .
$$

Note that we need to include the inverse $C^{-1}$ of the charge conjugation matrix $C$ in order to lower one index of the matrix $\gamma^{\mu}=\left(\gamma_{\alpha}^{\mu}{ }^{\beta}\right)$, see appendices A.1-A.3 for our index conventions.

\footnotetext{
${ }^{1}$ Depending on context, $V \cong \mathbb{R}^{t, s}$ denotes either a vector space, or the affine space modelled on this vector space (interpreted as a flat spacetime), or the Lie algebra of translations acting on the affine space.

${ }^{2}$ All standard facts about Clifford algebras and their relation to spin groups used in this paper can be found in [23].

${ }^{3}$ We use the term 'supersymmetry algebra' for the supertranslation algebra $V+\mathfrak{g}_{1}$, whose only non-trivial algebraic relation is the $Q-Q$ anti-commutator. As mentioned before, this is the only 'moving part' in our analysis once $\mathfrak{g}_{1}$ has been chosen.
} 
Since the bracket is symmetric, the right-hand side must be symmetric in the multi-indices $(i \alpha),(j \beta)$.

We will prefer to work with vector-valued bilinear forms on $\mathfrak{g}_{1}$, which allows us to suppress spinor indices. In this language the relation (1.4) is expressed using a bilinear form

$$
\beta:\left(S \otimes \mathbb{R}^{\mathcal{N}}\right) \times\left(S \otimes \mathbb{R}^{\mathcal{N}}\right) \rightarrow \mathbb{R}, \quad \beta(\lambda, \chi)=\left(\lambda^{i}\right)^{T} C \chi^{j} M_{j i},
$$

where we have written out the spinor module $\mathfrak{g}_{1}$ in terms of irreducible real $\operatorname{Spin}(t, s)$ modules $S$, which in our case are Majorana representations:

$$
\mathfrak{g}_{1}=S \otimes \mathbb{R}^{\mathcal{N}} \cong S \oplus \cdots \oplus S \quad(\mathcal{N}-\text { times }) .
$$

Since we take a sum of isomorphic modules, we can rewrite the $\mathcal{N}$-fold sum as a tensor product with an internal 'multiplicity space' $\mathbb{R}^{\mathcal{N}}$. Then the spin group only acts on the first factor but not on the internal space. Our strategy for disentangling the spin group and R-symmetry group will be to have the R-symmetry group acting only (or almost only) on the internal space, as we will discuss in more detail below.

The bilinear form $\beta$ is scalar-valued. Vector-valued bilinear forms, and, more generally, bilinear forms valued in antisymmetric tensors are obtained by substituting antisymmetrized products $\gamma^{(p)}=\gamma^{\mu_{1} \cdots \mu_{p}}=\gamma^{\left[\mu_{1}\right.} \cdots \gamma^{\left.\mu_{p}\right]}$ of $\gamma$-matrices into the first argument. In particular, the vector-valued bilinear form $\beta^{(1)}=\beta\left(\gamma^{\mu}, \cdot\right)$ allows us to express the superbracket (1.4) as

$$
\beta\left(\gamma^{\mu} \lambda, \chi\right)=\left(\gamma^{\mu} \lambda^{i}\right)^{T} C \chi^{j} M_{j i}
$$

To recover the superbracket (1.4) from (1.7) one expands the spinors $\lambda, \chi$ in a basis given by the supercharges, $\lambda=\lambda^{i \alpha} Q_{i \alpha}, \chi=\chi^{j \beta} Q_{j \beta}$. We refer to appendix A.3 for details. Up to isomorphism the superbracket only depends on a few invariants of the bilinear forms defined by the matrices $C=\left(C^{\alpha \beta}\right)$ and $M=\left(M_{i j}\right)$.

To define a superbracket, the vector-valued bilinear form $\beta\left(\gamma^{\mu}, \cdot\right)$ must be symmetric and equivariant (covariant) with respect to the spin group. As shown in [22] this is achieved by using admissible bilinear forms $\beta$ which are characterized by having a definite symmetry $\sigma_{\beta} \in\{1,-1\}$ and type $\tau_{\beta} \in\{1,-1\}$, where ${ }^{4}$

$$
\beta(\lambda, \chi)=\sigma_{\beta} \beta(\chi, \lambda), \beta\left(\gamma^{\mu} \lambda, \chi\right)=\tau_{\beta} \beta\left(\lambda, \gamma^{\mu} \chi\right) .
$$

The vector-valued bilinear form $\beta\left(\gamma^{\mu} \cdot, \cdot\right)$ is symmetric if $\sigma_{\beta} \tau_{\beta}=1$. Therefore such brackets $\beta$ will be called super-admissible. It can be shown that any super-admissible bracket $\beta$ on a spinor module $\mathfrak{g}_{1}$ defines a supersymmetry algebra. In particular the vectorvalued bracket is automatically spin-equivariant, and the super-Jacobi identity required to make $\mathfrak{s o}(V)+V+\mathfrak{g}_{1}$ a Lie superalgebra holds automatically. Conversely, the space of real, symmetric, spin-equivariant vector-valued bilinear forms, and, hence, the space of

\footnotetext{
${ }^{4}$ With regard to the symmetry, note that we work with commuting spinors in this paper. The translation to a formalism with anti-commuting spinors is straightforward and only introduces an additional sign. Using anti-commuting spinors is required when discussing properties of spinor bilinears in Lagrangians, a subject that we will not discuss in this paper. See, however, [16] where our formalism has been used to construct five-dimensional vector multiplets for arbitrary signature.
} 
real Poincaré Lie superalgebra structures related to a given spinor module $\mathfrak{g}_{1}$ is a finitedimensional real vector space which admits a basis given by forms of the type $\beta\left(\gamma^{\mu},, \cdot\right)$, where $\beta$ is super-admissible. In this sense all extended Poincaré Lie superalgebras are known for all dimensions and signatures. A basis of super-admissible bilinear forms has been constructed in [22]..$^{5}$ Since any linear combination of super-admissible bilinear forms defines a Poincaré Lie superalgebra (as long as it is non-degenerate, which is the generic case), superbrackets form continuous families. This raises the question of classification, that is to decide which brackets define non-isomorphic Poincaré Lie superalgebras. To address this question one needs to study the $\operatorname{Schur}$ group $\mathcal{C}^{*}\left(\mathfrak{g}_{1}\right)$, which is defined as the subgroup of automorphism group $\operatorname{Aut}\left(\mathfrak{g}_{1}\right)=\mathrm{GL}\left(\mathfrak{g}_{1}\right)$ of the real vector space $\mathfrak{g}_{1}$ whose action commutes with the action of $\mathfrak{s p i n}(V)$,

$$
\mathcal{C}^{*}\left(\mathfrak{g}_{1}\right)=\left\{Z \in \operatorname{Aut}\left(\mathfrak{g}_{1}\right) \mid[Z, \mathfrak{s p i n}(V)]=0\right\}=Z_{\mathrm{GL}\left(\mathfrak{g}_{1}\right)}(\mathfrak{s p i n}(V))
$$

that is, the centralizer of $\mathfrak{s p i n}(V) \subset \operatorname{GL}\left(\mathfrak{g}_{1}\right)$. Any two superbrackets which are in the same orbit of the action of the Schur group on the space of super-admissible bilinear forms are isomorphic. For later use we also define the Schur algebra $\mathcal{C}\left(\mathfrak{g}_{1}\right)=Z_{\operatorname{End}\left(\mathfrak{g}_{1}\right)}(\mathfrak{s p i n}(V))$, which is the centralizer of $\mathfrak{s p i n}(V)$ in the algebra $\operatorname{End}\left(\mathfrak{g}_{1}\right)$ of endomorphisms of $\mathfrak{g}_{1}$.

$$
\mathcal{C}\left(\mathfrak{g}_{1}\right)=\left\{Z \in \operatorname{End}\left(\mathfrak{g}_{1}\right) \mid[Z, \mathfrak{s p i n}(V)]=0\right\}=Z_{\mathrm{GL}\left(\mathfrak{g}_{1}\right)}(\mathfrak{s p i n}(V))
$$

The Schur group is the group of invertible elements of the Schur algebra.

The classification problem for Poincaré Lie superalgebras with odd part $\mathfrak{g}_{1}$ is almost, but not quite, equivalent to the problem of classifying the orbits of the Schur group on the space of superbrackets on $\mathfrak{g}_{1} .{ }^{6}$ The reason is that elements of the pin group $\operatorname{Pin}(V)$ which are not contained in the spin group $\operatorname{Spin}(V)$ may lead to isomorphisms between brackets which belong to different orbits. Recall that $\operatorname{Pin}(V)$ is a double cover of the full orthogonal group $\mathrm{O}(V)$, while $\operatorname{Spin}(V)$ is a double cover of the special orthogonal group $\mathrm{SO}(V)$. Both groups are contained in the Clifford algebra $C l(V)$. A precise criterion for two Poincaré Lie superalgebras to be isomorphic is given by Theorem 1 of [17]. As illustrated in [17] by the classification of four-dimensional supersymmetry algebras with eight real supercharges for arbitrary signature, this classification can be done case by case but requires some work. However there is a sufficient condition for two supersymmetry algebras to be non-isomorphic which is easier to check, namely that their R-symmetry groups are different. We define the $R$-symmetry group of a superbacket as the subgroup of the Schur group under which this superbracket invariant (its stabilizer group):

$$
G_{R}=\left\{R \in \mathcal{C}^{*}\left(\mathfrak{g}_{1}\right) \mid \beta\left(\gamma^{\mu} R \cdot R \cdot\right)=\beta\left(\gamma^{\mu} \cdot, \cdot\right)\right\} .
$$

Note that our definition does not depend on how the R-symmetry group acts on the fields in particular field theoretic realizations of the algebra. Moreover, with our definition the

\footnotetext{
${ }^{5}$ One can also include poly-vector charges (BPS charges), that is additional terms in the supersymmetry anti-commutator which transform as antisymmetric Lorentz tensors [24]. The inclusion of such charges in our formalism will be left for future work.

${ }^{6}$ We will use the terms super-admissible bilinear form and superbracket interchangeably.
} 
R-symmetry group is not necessarily a connected group. This may lead to slight differences when comparing our tables to the literature.

We will also need to consider the complexification $\mathfrak{g}^{\mathbb{C}}$ of a Poincaré Lie superalgebra. In this context one can define complex versions of the Schur algebra, Schur group and R-symmetry group, which will be denoted $\mathcal{C}_{\mathbb{C}}\left(\mathfrak{g}_{1}^{\mathbb{C}}\right), \mathcal{C}_{\mathbb{C}}^{*}\left(\mathfrak{g}_{1}^{\mathbb{C}}\right)$, and $G_{R}^{\mathbb{C}}$.

\subsection{Overview of the construction}

To explain the main idea of our construction, recall how supersymmetry can be formulated in terms of symplectic Majorana spinors. In signature $(1,3)$ the unique (up to isomorphism) irreducible real spinor representation is the Majorana representation, and the smallest or $\mathcal{N}=1$ supersymmetry algebra is based on supercharges $Q_{\alpha}$ which are Majorana spinors. The standard $\mathcal{N}=2$ algebra can be written in terms of two Majorana spinors, or of a single Dirac spinor, but there also is a third option, namely to take two Dirac spinors $Q_{i \alpha}$, and to impose a reality condition. The supersymmetry algebra is then defined by a complex superbracket, subject to a reality condition: ${ }^{7}$

$$
\left\{Q_{i \alpha}, Q_{j \beta}\right\}=M_{i j}\left(\gamma^{\mu} C^{-1}\right)_{\alpha \beta} P_{\mu}, \quad\left(Q^{i}\right)^{*}=\alpha B Q^{j} L_{j i}, \quad i, j=1,2,
$$

where in our specific case $M_{i j}=L_{i j}=\epsilon_{i j}$, the charge conjugation matrix $C$ is antisymmetric, and $B$ satisfies $B B^{*}=-\mathrm{Id}$, indices $i, j$ are raised and lowered using $\epsilon_{i j}, \epsilon^{i j}$, and $\alpha$ is a conventional phase. A pair of Dirac spinors $\lambda^{i}$ which satisfies

$$
\left(\lambda^{i}\right)^{*}=\alpha B \lambda^{j} \epsilon_{j i}
$$

is called a pair of symplectic Majorana spinors.

The same construction can be applied in signature $(1,4)$, where Majorana spinors do not exist and Dirac spinors are irreducible, so that the smallest supersymmetry algebra has eight real supercharges. As in signature $(1,3)$ one can replace a Dirac spinor by a pair of symplectic Majorana spinors, with the supersymmetry algebra taking the form (1.12). While one also can express the supersymmetry algebra in terms of Dirac spinors (see for example [12]), the formulation using symplectic Majorana spinors is the standard one in five dimensions. One of its advantages is its manifest R-symmetry: the group which leaves both the complex superbracket and the reality condition invariant is $\operatorname{USp}(2, \mathbb{R}) \cong \mathrm{SU}(2)$.

Formuling the supersymmetry algebra in terms of symplectic Majorana spinors can be interpreted as complexifying the space of Dirac spinors $\mathbb{S}$, and then imposing a reality condition. The space $\mathbb{S}$ of Dirac spinors, which is also called the complex spinor module, is obtained by restricting an irreducible representation of the complex Clifford algebra $C l\left(V^{\mathbb{C}}\right)$, where $V^{\mathbb{C}}=V \otimes_{\mathbb{R}} \mathbb{C}$, to the real spin group $\operatorname{Spin}(V)$. The complexification of $\mathbb{S}$, regarded as a real spin module, is $\mathbb{S}^{\mathbb{C}}=\mathbb{S} \otimes_{\mathbb{R}} \mathbb{C}$. Since $\mathbb{S}$ admits a spin invariant complex structure, its complexification is self-conjugate, $\mathbb{S}^{\mathbb{C}}=\mathbb{S} \oplus \overline{\mathbb{S}}$, where $\overline{\mathbb{S}}$ is the complex-conjugate module. Moreover, in any dimension and signature one can find a $\operatorname{Spin}(t, s)$ invariant matrix $B$

\footnotetext{
${ }^{7}$ When formulating this algebra using Majorana spinors, one uses a different charge conjugation matrix $C^{\prime}$, which is symmetric, and a different matrix $B^{\prime}$, which satisfies $B^{\prime} B^{\prime *}=+\mathrm{Id}$. See tables 1 and 2 for information about the properties of the matrices $C$ and $B$ in arbitrary dimension and signature.
} 
acting on $\mathbb{S}$ which satisfies $B B^{*}= \pm \mathrm{I}$. Therefore the complex spinor module $\mathbb{S}$ always carries either an invariant real structure or an invariant quaternionic structure, and thus is self-conjugate, $\mathbb{S} \cong \overline{\mathbb{S}}$ as $\operatorname{Spin}(t, s)$ as module. Therefore

$$
\mathbb{S}^{\mathbb{C}}=\mathbb{S} \otimes_{\mathbb{R}} \mathbb{C}=\mathbb{S} \oplus \overline{\mathbb{S}} \cong \mathbb{S} \oplus \mathbb{S} \cong \mathbb{S} \otimes_{\mathbb{C}} \mathbb{C}^{2}
$$

In the last step we have rewritten the sum $\mathbb{S} \oplus \mathbb{S}$ as a complex tensor product, where the second factor is an auxiliary multiplicity space which encodes that we have two copies of $\mathbb{S}$. This corresponds to the expression for the complex superbracket in (1.12) where we use a pair of Dirac spinors $Q_{i \alpha}$, with the index $i=1,2$ referring to the multiplicity space $\mathbb{C}^{2}$.

The complex superbracket (1.12) defines a complex Poincaré Lie superalgebra $\mathfrak{s o}\left(V^{\mathbb{C}}\right) \oplus$ $V^{\mathbb{C}} \oplus \mathfrak{g}_{1}^{\mathbb{C}}$, where $V^{\mathbb{C}}=V \otimes_{\mathbb{R}} \mathbb{C}$ is the complexified translation algebra, $\mathfrak{s o}\left(V^{\mathbb{C}}\right)$ the complexified Lorentz Lie algebra and $\mathfrak{g}_{1}^{\mathbb{C}}=\mathbb{S}^{\mathbb{C}}$ the complexified spinor module. By imposing the reality condition (1.12) we recover the real supersymmetry algebra as a real form of this complex algebra.

We already mentioned that the multiplicity space $\mathbb{C}^{K}=\mathbb{C}^{2}$ is useful in making explicit the action of the R-symmetry group. In five dimensions, where $\mathbb{S}$ is irreducible, the Schur group acts trivially on the factor $\mathbb{S}$ in $\mathbb{S} \otimes_{\mathbb{C}} \mathbb{C}^{2}$ and therefore the complex Schur group is $\mathrm{GL}(2, \mathbb{C}) .{ }^{8}$ The R-symmetry group of the complex supersymmetry algebra is the subgroup which in addition preserves the bilinear form defined by $M_{i j}=\epsilon_{i j}$ on $\mathbb{C}^{2}$, that is $G_{R}^{\mathbb{C}}=$ $\operatorname{Sp}(2, \mathbb{C})$. The R-symmetry group $G_{R}$ of the real supersymmetry algebra is the real form of $G_{R}^{\mathbb{C}}$ which also preserves the reality condition in (1.12), that is $G_{R}=\mathrm{USp}(2, \mathbb{R}) \cong \operatorname{SU}(2)$. In four dimension, $\mathbb{S}$ becomes reducible, which enlarges the R-symmetry group to $\mathrm{U}(2) .^{9}$

The observation that we will expand on in this paper is that the construction of real supersymmetry algebras based on symplectic Majorana spinors can be adapted to arbitrary dimension and signature. This requires of course to also consider other types of reality conditions. Extended real supersymmetry algebras are included by enlarging the internal multiplicity space.

Thus, schematically, the idea is:

$$
\text { Real supersymmetry algebra } \leftrightarrow\left\{\begin{array}{l}
\text { Complex bilinear form (superbracket), } \\
\text { Reality condition. }
\end{array}\right.
$$

Depending on the signature, there either is a unique (up to isomorphism) irreducible real spinor representation given by Dirac or Majorana spinors, or there are two inequivalent ones, given by Weyl spinors or Majorana-Weyl spinors. For simplicity we first explain how our construction works when the irreducible real spinor module is unique.

1. We start with a complex supersymmetry algebra with spinor module given by $K$ copies of the complex spinor module, $\mathfrak{g}_{1}^{\mathbb{C}}=\mathbb{S} \otimes \mathbb{C}^{K}$. We will sometimes refer to this as the $K$-extended spinor module, or extended spinor module for short. To define a complex

\footnotetext{
${ }^{8}$ To be precise, by Schur's lemma the R-symmetry group acts on $\mathbb{S}$ by scalar multiplication, which we can absorb into its action on $\mathbb{C}^{2}$.

${ }^{9}$ We will explain how to deal with this in due course. In appendix D we present two methods which allow to disentangle the spin and R-symmetry groups.
} 
supersymmetry algebra, we need to specify a complex super-admissible bilinear form $\beta$. Since the Gram matrix of such a bilinear form has precisely the properties of a charge conjugation matrix $C$, this amounts to choosing a charge conjugation matrix. If the bilinear form is super-admissible we can extend it to $\mathbb{S} \otimes \mathbb{C}^{K}$ as $\beta=C \otimes \delta$, where $\delta$ is the complex bilinear form on $\mathbb{C}^{K}$ defined by the unit matrix. If $C$ is not super-admissible, we can still obtain a super-admissible bilinear form on $\mathbb{S} \otimes \mathbb{C}^{K}$ for $K$ even, if we extend $C$ as $\beta=C \otimes \epsilon$, where $\epsilon$ defines a non-degenerate anti-symmetric bilinear form. Charge conjugation matrices are classified by their symmetry $\sigma$ and type $\tau$. In odd dimensions charge conjugation matrices are unique up to isomorphism, while in even dimensions there are two inequivalent ones, denoted $C_{ \pm}=C_{-\tau}$, which are of opposite type $\tau$, see table 2. We can now write down the possible complex supersymmetry algebras in any dimension, and for any value of $K$, and determine their Schur groups and R-symmetry groups.

2. Then we choose a signature $(t, s)$ and a $\operatorname{Spin}(t, s)$ invariant reality condition on $\mathfrak{g}_{1}^{\mathbb{C}}=\mathbb{S} \otimes \mathbb{C}^{K}$. In odd dimensions $\mathbb{S}$ admits either a spin invariant real structure or a spin invariant quaternionic structure, defined using the matrix $B$ which relates the $\gamma$-matrices to their complex conjugates. In even dimensions there are two nonequivalent such matrices, $B_{ \pm}$, and, depending on signature, $\mathbb{S}$ admits two inequivalent real structures or two inequivalent quaternionic structures, or one of each type. We emphasize that while $B_{ \pm}$can be constructed out of $C_{ \pm}$, the choice of a reality condition is independent of the choice of the bilinear form on $\mathbb{S}$, that is, there are four possible combinations of $C_{ \pm}$with $B_{ \pm}$in our formalism. To define a real supersymmetry algebra we need a real structure on $\mathbb{S} \otimes \mathbb{C}^{K}$, which can be obtained either as a product of two real structures or of two quaternionic structures on the factors. The real or quaternionic structure on $\mathbb{C}^{K}$ is chosen such that it defines a real form $G_{R}$ of the complex R-symmetry group $G_{R}^{\mathbb{C}}$, that is, we construct it using an involutive automorphism of the Lie algebra of $G_{R}^{\mathbb{C}}$. We can then list, for all dimensions and signatures, the real supersymmetry algebras that result from a consistent pairing of complex superbrackets with reality conditions, and determine their R-symmetry groups.

There are two additional issues in even dimensions, where Dirac spinors are reducible and decompose into Weyl spinors, $\mathbb{S} \cong \mathbb{S}_{+} \oplus \mathbb{S}_{-}$.

- Firstly, there are signatures with two inequivalent irreducible real spinor representations, which can be either Weyl spinors or Majorana-Weyl spinors. If this happens there potentially are supersymmetry algebras based on spinor modules of the form $\mathfrak{g}_{1}^{\mathbb{C}}=\mathbb{S}_{+} \otimes \mathbb{C}^{K_{+}} \oplus \mathbb{S}_{-} \otimes \mathbb{C}^{K_{-}}$, where $K_{ \pm}$can be chosen independently. ${ }^{10}$ The existence of such chiral supersymmetry algebras requires more than the existence of inequivalent

\footnotetext{
${ }^{10}$ As it is clear from context that $\mathbb{S}_{+}$and $\mathbb{C}^{K_{+}}$are considered as complex modules, we have written $\otimes$ rather than $\otimes_{\mathbb{C}}$. Similarly, we will simply write $\otimes$ in the future when the tensor product is between a complex spin representation and an auxiliarly complex multiplicity space, which encodes copies of equivalent representations.
} 
irreducible real spinor modules. Firstly, there must exist a superbracket which pairs $\mathbb{S}_{+}$with $\mathbb{S}_{+}$and $\mathbb{S}_{-}$with $\mathbb{S}_{-}$, while vanishing between $\mathbb{S}_{+}$and $\mathbb{S}_{-}$. This can be decided using an invariant of the charge conjugation matrix $C$, its isotropy $\iota$. Superbrackets are either orthogonal, $\iota=1$, which means that they are non-degenerate on $\mathbb{S}_{+} \times \mathbb{S}_{+}$ and $\mathbb{S}_{-} \times \mathbb{S}_{-}$but vanish on $\mathbb{S}_{+} \times \mathbb{S}_{-}$and $\mathbb{S}_{-} \times \mathbb{S}_{+}$, or they are isotropic, $\iota=-1$, which means that they are non-degenerate on $\mathbb{S}_{+} \times \mathbb{S}_{-}$and $\mathbb{S}_{-} \times \mathbb{S}_{+}$but vanish on $\mathbb{S}_{+} \times \mathbb{S}_{+}$ and $\mathbb{S}_{-} \times \mathbb{S}_{-}$. The isotropy of a superbracket only depends on the dimension, and we will see that superbrackets are orthogonal in dimensions $2,6,10, \ldots$. An orthogonal complex superbracket is necessary, but not sufficient for a chiral supersymmetry algebra to exist, because in order to obtain a real superbracket we also need to impose a reality condition. A reality condition can either respect chirality, in which case we call it 'Weyl compatible' or it can flip it, in which case we call it 'Weyl incompatible'. Weyl compatibility is a signature dependent property, and therefore the existence of chiral supersymmetry algebras is signature dependent.

Chiral supersymmetry algebras require both an orthogonal complex bilinear form and a Weyl compatible reality condition, and therefore Weyl (in-)compatibility is a signature dependent property.

- Secondly, since $\mathbb{S}$ is reducible the R-symmetry acts non-trivially on $\mathbb{S}$. However it acts irreducibly, and therefore as a multiple of the identity Id on the complex irreducible Weyl spinor modules $\mathbb{S}_{+}$and $\mathbb{S}_{-}$. This can be used to determine the $\mathrm{R}$-symmetry group. The details depend on whether the complex bilinear form is orthogonal or isotropic, and on whether the reality condition is Weyl compatible or Weyl incompatible. Since there are four cases to consider, the classification of R-symmetry is somewhat involved in even dimensions. Using a certain block matrix notation one can manifestly disentangle the actions of the spin and R-symmetry groups.

\subsection{Organisation of the paper}

The program described above is carried out as follows in the bulk of this paper.

- In section 2 we provide the necessary background on Clifford and spin representations, with further details relegated to appendices A, B and C.

- In section 3 we obtain complex supersymmetry algebras by constructing superadmissible bilinear forms on the complexified extended spinor module $\mathfrak{g}_{1}^{\mathbb{C}}$, where $\mathfrak{g}_{1}^{\mathbb{C}}=\mathbb{S} \otimes \mathbb{C}^{K}$ or $\mathfrak{g}_{1}^{\mathbb{C}}=\mathbb{S}_{+} \otimes \mathbb{C}^{K_{+}} \oplus \mathbb{S}_{-} \otimes \mathbb{C}^{K_{-}}$. To do so we first have to study admissible bilinear forms on the spaces $\mathbb{S}$ and $\mathbb{S}_{ \pm}$of Dirac and Weyl spinors, and bilinear forms on $\mathbb{C}^{K}$. The resulting complex supersymmetry algebras are classified by their R-symmetry groups which are listed in table 2 . This table contains all relevant information about the superbrackets that we use in our construction.

- In section 4 we systematically construct spin invariant reality conditions on $\mathfrak{g}_{1}^{\mathbb{C}}$. These are built out of real and quaternionic structures on $\mathbb{S}, \mathbb{S}_{ \pm}$and $\mathbb{C}^{K}$. To discuss real and quaternionic structures in parallel, we use the unifying concept of an $\epsilon$-quaternionic 
structure. For dimensions up to twelve, and for all signatures, table 1 lists all inequivalent real and quaternionic structures on the complex spinor module $\mathbb{S}$, and their properties. This table encodes all relevant information about reality conditions used in our formalism, and, in particular, allows to read off the Schur algebras $\mathcal{C}(\mathbb{S})$.

- In section 5 we analyze how reality conditions can be imposed consistently on complex superbrackets in order to define real supersymmetry algebras.

- In section 6 we classify the real R-symmetry groups which arise in our construction. Table 3 shows how the real forms of the complex R-symmetry groups listed in table 2 arise from reality conditions. Table 4 lists all possible real R-symmetry groups in odd dimensions up to 11 . In even dimensions the possible supersymmetry algebras and R-symmetry groups fall into four classes, depending on whether the bilinear form is orthogonal or isotropic, and whether the reality condition is Weyl-compatible or Weylincompatible. In orthogonal Weyl-incompatible signatures the real R-symmetry group only depends on the dimension, while the real R-symmetry groups for orthogonal, Weylcompatible signatures are listed in table 5 and for isotropic signatures with either reality condition in table 6 . For reference and convenience, we also provide a master table, table 9, for general even dimensions and a master table, table 10, for all dimensions.

- In section 7 we explain why real supersymmetry algebras constructed by our method are classified by their R-symmetry group, together with the choice of a relative sign for chiral supersymmetry algebras. Details about the isomorphisms required to show this have been relegated to appendix E.

- In section 8 we apply our results to study supersymmetry algebras of type-II string theories in dimensions 10 and 9 and of their Calabi-Yau compactifications to dimensions 4 and 3. We show how our formalism allows one to identify which theories exist in a given signature, and how to determine their mutual relations by spacelike and timelike reduction and T-duality. For four-dimensional $\mathcal{N}=2$ theories and three-dimensional $\mathcal{N}=4$ theories we explain how the geometry of the scalar manifold can be read off from the R-symmetry group. Tables 12, 13 and 14 provide summaries.

- In section 9 we make some concluding remarks and provide an outlook onto open questions and future work.

In order to keep the bulk of this paper as short as possible, some details have been relegated to appendices, together with additional background information that is helpful but would have interrupted the flow of the main narrative. In appendix A we summarize our conventions and notation, and in particular explain how the (partially) index free notation based on bilinear forms that we use in the main part of the paper relates to the standard notation using anti-commutators of supercharges. We also list various formulae which are used in the main part of this paper. In appendix B we explain the relation between real spinors and real semi-spinors, as defined in the mathematics literature, to Majorana, Weyl and Majorana-Weyl spinors, as defined in the physics literature. In appendix $\mathrm{C}$ we present 
details on the complexification of spinor modules, which are relevant for understanding the precise relation between the odd parts $\mathfrak{g}_{1}$ and $\mathfrak{g}_{1}^{\mathbb{C}}$ of real and complex supersymmetry algebras. In appendix D we review the matrix notation for Weyl spinors introduced in [17], which is used in the main part to disentangle the actions of the spin and R-symmetry group in even dimensions by doubling the auxiliary multiplicity space of our construction. Alternatively one can work without doubling, in which case the R-symmetry group operates on spinor indices, in a way that we also explain in appendix D. In appendix E we provide the details of several isomorphisms which are needed to show that the supersymmetry algebras that we have constructed are classified, essentially, by their R-symmetry groups. In appendix F we collect formulae which allow one to carry out the spacelike and timelike dimensional reduction of spinors, superbrackets and reality conditions.

\section{Clifford and spinor representations}

In this section we review the necessary background on Clifford algebras, spin groups and their representations for arbitrary signature. Our presentation is partially based on [25], whose conventions we follow except for some tweaks where we follow [12, 22, 24]. We will also use certain facts about Clifford algebras and real associative algebras, see for example $[23,26]$.

\subsection{Clifford and spinor modules}

We consider flat space-times $V \cong \mathbb{R}^{t, s}$ of arbitrary signature $(t, s)$ and dimension $D=t+s$. Our convention for the metric is $\eta=\operatorname{diag}(-1, \ldots,-1,1, \ldots, 1)$ with $t$ entries -1 . The associated Clifford algebras $C l_{t, s}$ are the real algebras with generators $\gamma^{\mu}, \mu=1, \ldots, n$ and relations

$$
\left\{\gamma^{\mu}, \gamma^{\nu}\right\}=2 \eta^{\mu \nu} \mathbb{1}
$$

Clifford algebras are real, associative algebras with a unit, and isomorphic to real, complex or quaternionic matrix algebras of the form $\mathbb{R}(n), \mathbb{C}(n), \mathbb{H}(n), 2 \mathbb{R}(n), 2 \mathbb{H}(n)$, where $\mathbb{K}(n)$ is the algebra of $n \times n$ matrices over $\mathbb{K}=\mathbb{R}, \mathbb{C}, \mathbb{H}$, where $2 \mathbb{K}(n):=\mathbb{K}(n) \oplus \mathbb{K}(n)$, and where $n=2^{\left[\frac{D}{2}\right]}$. By allowing complex linear combinations of the generators $\gamma^{\mu}$ one obtains the complex Clifford algebras $\mathbb{C} l_{t+s}=C l_{t, s} \otimes_{\mathbb{R}} \mathbb{C}$, which are isomorphic to matrix algebras of the form $\mathbb{C}(n)$ in even dimensions and to matrix algebras of the form $2 \mathbb{C}(n)=\mathbb{C}(n) \oplus \mathbb{C}(n)$ in odd dimensions. ${ }^{11}$

Algebras of the form $m \mathbb{K}(n)$ have $m$ inequivalent irreducible representations, where one of the summands $\mathbb{K}(n)$ acts on $\mathbb{K}^{n}$ by matrix multiplication, while the other summands act trivially. Therefore Clifford algebras have either one to two inequivalent irreducible representations. Clifford representations give rise to spinor representations by restriction, because the real and complex spin groups are naturally embedded into the real and complex Clifford algebras. The even subalgebra $C l_{t, s}^{0} \subset C l_{t, s}$ of the real Clifford algebra is the subalgebra which is generated by even products of the generators $\gamma^{\mu}$. This subalgebra is itself a Clifford algebra. The spin group $\operatorname{Spin}(t, s)$ is the subgroup of (the group of invertible

\footnotetext{
${ }^{11}$ See for example tables I and II of [23] for a complete list of Clifford algebras as matrix algebras.
} 
elements of) $C l_{t, s}^{0}$ which is generated by unit norm elements. Therefore irreducible $C l_{t, s}^{0}$ modules become irreducible $\operatorname{Spin}(t, s)$ modules by restriction. The real spinor module $S$ is the $\operatorname{Spin}(t, s)$ module obtained by restricting an irreducible $C l_{t, s}$ module. As a real spin representation, $S$ is either irreducible, or decomposes into two irreducible real semi-spinor modules $S_{ \pm}, S=S_{+}+S_{-}$. The real semi-spinor modules can be isomorphic to each other, $S_{+} \cong S_{-}$or non-isomorphic, $S_{+} \cong S_{-}$. One can decide which of these three cases is realised by comparing the matrix algebras realizing $C l_{t, s}$ and $C l_{t, s}^{0}$, and using that $\mathbb{K}(n)$ has one, while $2 \mathbb{K}(n)$ has two inequivalent irreducible representations. We can also determine the Schur algebras $\mathcal{C}(S)$ and $\mathcal{C}\left(S_{ \pm}\right)$by using corrolaries to Schur's Lemma, see [26]. For the case at hand, the relevant statements are:

- If $\Sigma=\mathbb{K}^{n}$ is an irreducible module of the real spin group $\operatorname{Spin}(t, s) \subset C l_{t, s}^{0}$, then its Schur algebra is $\mathbb{K}^{12}$

- If $\Sigma_{1} \oplus \Sigma_{2}$ is the sum of two irreducible modules of the real spin group $\operatorname{Spin}(t, s) \subset C l_{t, s}^{0}$, then the Schur algebra is $2 \mathbb{K}$ if $\Sigma_{1} \nsucceq \Sigma_{2}$ and $\mathbb{K}(2)$ if $\Sigma_{1} \cong \Sigma_{2}$.

The complex spin group $\operatorname{Spin}(t+s, \mathbb{C})$ is the subgroup of unit norm elements of the even subalgebra $\mathbb{C} l_{t+s}^{0} \subset \mathbb{C} l_{t+s}$. The complex spinor module $\mathbb{S}$ is the $\operatorname{Spin}(t, s)$-module obtained by restricting an irreducible $\mathbb{C l}_{t+s}$-module. Its elements are the Dirac spinors. Since $\mathbb{C} l_{t+s}^{0} \cong \mathbb{C} l_{t+s-1}$, it follows that $\mathbb{S}$ is irreducible in odd dimensions, but decomposes into two irreducible complex semi-spinor modules $\mathbb{S}_{ \pm}$in even dimensions, $\mathbb{S}=\mathbb{S}_{+}+\mathbb{S}_{-}$. The elements of $\mathbb{S}_{ \pm}$are also called Weyl spinors. Note that while $\mathbb{S}_{ \pm}$are non-isomorphic as $\mathbb{C} l_{t+s}^{0}$ modules, they may or may not be isomorphic as real $\operatorname{Spin}(t, s)$-modules. The decomposition of $\mathbb{S}$ into irreducible $\operatorname{Spin}(t, s)$-modules can be obtained by comparing the matrix algebras realizing $\mathbb{C l}_{t+s}, C l_{t, s}$ and $C l_{t, s}^{0}$. This also allows one to determine the complex Schur algebras $\mathcal{C}_{\mathbb{C}}(\mathbb{S}), \mathcal{C}_{\mathbb{C}}\left(\mathbb{S}_{ \pm}\right)$, and the real Schur algebras $\mathcal{C}(\mathbb{S}), \mathcal{C}\left(\mathbb{S}_{ \pm}\right)$. The complex Schur algebra of an irreducible representation of $\operatorname{Spin}(t+s, \mathbb{C}) \subset \mathbb{C} l_{t+s}^{0}$ is $\mathbb{C}$, while the complex Schur algebra of the sum of two irreducible complex representations is $2 \mathbb{C}$ if the representations are not equivalent and $\mathbb{C}(2)$ if they are equivalent.

As an example, consider the case where $(t, s)=(1,3)$. Then $\mathbb{C} l_{4}=\mathbb{C}(4)$ and $\mathbb{C} l_{4}^{0}=2 \mathbb{C}(2)$ which implies $\mathbb{S}=\mathbb{C}^{4}$ and $\mathbb{S}_{ \pm}=\mathbb{C}^{2}$ which are the Dirac and Weyl spinors respectively. Since $\mathbb{S}_{ \pm}$are complex irreducible, their complex Schur algebras are $\mathcal{C}_{\mathbb{C}}\left(\mathbb{S}_{ \pm}\right) \cong \mathbb{C}$, and since they are inequivalent as complex modules, $\mathcal{C}_{\mathbb{C}}(\mathbb{S})=2 \mathbb{C}$. Since $C l_{1,3}=\mathbb{R}(4)$, the real spinor module is $S=\mathbb{R}^{4}$. This implies that $\mathbb{S}=S \otimes_{\mathbb{R}} \mathbb{C}$ and shows that in this case real spinors are Majorana spinors, that is, they arise by imposing a reality condition on Dirac spinors. The even part of the real Clifford algebra is $C l_{1,3}^{0}=\mathbb{C}(2)$ and since $\mathbb{C}^{2} \cong \mathbb{R}^{4}$, real spinors are irreducible. There are no real semi-spinors (which would be Majorana-Weyl spinors), and Majorana spinors are equivalent, as real spin representations, to Weyl spinors $S \cong \mathbb{S}_{ \pm} \cdot{ }^{13}$ The Schur

\footnotetext{
${ }^{12}$ The cases where $\mathbb{K}=\mathbb{R}, \mathbb{C}, \mathbb{H}$ correspond to what is usually called real, complex and quaternionic representations, respectively. In all cases we are interested in the underlying real representation. Complex and quaternionic representations are thus viewed as real representations with additional invariant structures that are encoded by the Schur algebra.

${ }^{13}$ This is reflected by the familiar fact that in this case the $\mathcal{N}=1$ supersymmetry algebra can be equivalently expressed using Majorana spinor or Weyl spinors.
} 
algebra of the real spinor module $S=\mathbb{C}^{2}$ is $\mathcal{C}_{1,3}(S)=\mathbb{C}$. Since $\mathbb{S}_{ \pm}$are equivalent as real spin modules, the Schur algebra of $\mathbb{S}$, considered as a real spin module, is $\mathcal{C}_{1,3}(\mathbb{S})=\mathbb{C}(2)$.

It is instructive to compare this to the case $(t, s)=(3,1)$ which is Minkowski space with a mostly minus convention for the metric. In this case the real Clifford algebra is $C l_{3,1}=\mathbb{H}(2)$ and therefore the real spinor module is $S=\mathbb{H}^{2} \cong \mathbb{C}^{4}=\mathbb{S}$. Now real spinors are Dirac spinors, and because $C l_{3,1}^{0}=\mathbb{C}(2)$ they are reducible and decompose into real semi-spinors, $S=S_{+} \oplus S_{-}$, where $S_{ \pm}=\mathbb{C}^{2} \cong \mathbb{R}^{4}$. For dimensional reasons $\mathbb{S}_{ \pm} \cong S_{ \pm}$. Since $C l_{3,1}^{0} \cong \mathbb{C}(2)$ only has one inequivalent irreducible representation, $S_{ \pm}$are isomorphic as real spin modules. Therefore the Schur algebra of $\mathbb{S}=S$ is $\mathcal{C}_{3,1}(\mathbb{S})=\mathcal{C}_{3,1}(S)=\mathbb{C}(2)$. In summary, real semi-spinors and complex semi-spinors are equivalent to each other and correspond to Weyl spinors of either chirality, which are equivalent, as real spin representations, to Majorana spinors. ${ }^{14}$

For more examples we refer to $[16,17]$ where the spinor modules and Schur algebras have been worked for $D=4,5$ for all signatures. This already provides examples of all possible cases.

\subsection{Spinor representations in arbitrary signature}

The Clifford generators can be realized explicity as $\gamma$-matrices, which like the abstract generators we denote by $\gamma^{\mu}$. These are complex $2^{\left[\frac{D}{2}\right]} \times 2^{\left[\frac{D}{2}\right]}$ matrices and can be constructed as tensor products of the Pauli matrices and the $2 \times 2$ identity matrix, see for example [25]. The $\gamma$-matrices generate a representation of the real Clifford algebra $C l_{t, s}$ on $\mathbb{C}^{\left[\frac{D}{2}\right]}$, which extends to an irreducible representation of its complexification $\mathbb{C l}_{t+s}$. By restriction to $\operatorname{Spin}(t, s)$, this becomes the complex spinor module $\mathbb{S}$ introduced above. Its elements are the Dirac spinors.

The $\gamma$-matrices can be changed by equivalence transformations, which can be used to select representations which are convenient for performing computations. We impose that timelike $\gamma$-matrices are anti-Hermitian, while spacelike $\gamma$-matrices are Hermitian:

$$
\left(\gamma^{\mu}\right)^{\dagger}=\left\{\begin{aligned}
-\gamma^{\mu}, \mu & =1, \ldots, t \\
\gamma^{\mu}, & \mu=t+1, \ldots t+s=D
\end{aligned}\right.
$$

The remaining freedom of performing unitary transformation will be used later to impose further conditions. In odd dimensions the product $\omega=\gamma_{1} \cdots \gamma_{D}$ commutes with all generators $\gamma_{\mu}$ and distinguishes between the two inequivalent representations of $C l_{t, s}$ (which define equivalent spin representations). In even dimensions $\omega$ anti-commutes with all generators, and commutes with the spin generators $\gamma^{\mu \nu}=\frac{1}{2}\left[\gamma^{\mu}, \gamma^{\nu}\right]$. Therefore $\omega$ can be used to decompose the complex spinor module $\mathbb{S}$ into complex semi-spinor modules $\mathbb{S}_{ \pm}$. Depending on signature, $\omega^{2}=(-1)^{t} \mathbb{1}$ or $\omega^{2}=(-1)^{t+1} \mathbb{1}$. Setting $\gamma_{*}= \pm \omega$ or $\gamma_{*}= \pm i \omega$ one obtains an operator which satisfies $\gamma_{*}^{2}=1$ and therefore can be used to define projectors $\frac{1}{2}\left(1 \pm \gamma_{*}\right)$ onto the complex semi-spinor modules. For Lorentz signature $(1, D-1)$ (or, using a 'mostly minus' convention $(D-1,1)) \gamma_{*}$ is the chirality operator which defines Weyl spinors. We will therefore refer to complex semi-spinors as Weyl spinors. For computational

\footnotetext{
${ }^{14}$ We do not distinguish between Majorana and Pseudo-Majorana spinors, but see for example [2].
} 
purposes it is convenient to fix the relation between $\gamma_{*}$ and $\omega$. We choose

$$
\gamma_{*}=(-i)^{\frac{D}{2}+t} \gamma_{1} \ldots \gamma_{D}
$$

One can always construct matrices $A, B, C$ which relate the $\gamma$-matrices to their Hermitian conjugates, complex conjugates, and transposed, respectively:

$$
\begin{aligned}
\left(\gamma^{\mu}\right)^{\dagger} & =(-1)^{t} A \gamma^{\mu} A^{-1}, \\
\left(\gamma^{\mu}\right)^{*} & =(-1)^{t} \tau B \gamma^{\mu} B^{-1}, \\
\left(\gamma^{\mu}\right)^{T} & =\tau C \gamma^{\mu} C^{-1},
\end{aligned}
$$

where $\tau= \pm 1$, with the allowed values depending on the dimension $D .{ }^{15}$ One possible choice for $A$ is the product $\gamma_{1} \cdots \gamma_{t}$ of all timelike $\gamma$-matrices. In Lorentz signature $(1, D-1)$, this gives the usual $A=\gamma_{0}$, where we have shifted the range of Lorentz indices to $\mu=0, \ldots, D-1 .{ }^{16}$ We remark that our choice for $A$ is not unique, and for Lorentz signature there are conventions which differ from ours by a factor -1 or $\pm i$.

The matrix $C$ is the charge conjugation matrix. One can always choose a representation where $C$ is Hermitian and unitary,

$$
C^{\dagger}=C^{-1}=C
$$

$C$ is either symmetric or antisymmetric

$$
C^{T}=\sigma C
$$

where $\sigma= \pm 1 .{ }^{17}$ Which values of $\tau$ and $\sigma$ are possible depends on the dimension $D$, and we have listed these values in table 2 . The well known periodicity modulo 8 of the classification of real Clifford algebras implies that this table is periodic modulo 8 , so that it encodes the values for arbitrary dimension. While in odd dimensions there is only one charge conjugation matrix up to equivalence, there are two inequivalent choices in even dimensions, which are distinguished by the corresponding value of $\tau$,

$$
C_{ \pm}:=C_{-\tau}
$$

Both charge conjugation matrices are related through multiplication by the chirality matrix $\gamma_{*}$ :

$$
C_{ \pm}=\gamma_{*} C_{\mp} .
$$

\footnotetext{
${ }^{15} \tau$ is related to the parameter $\eta$ in [25] by $\tau=-\eta$. Note that some authors, for example [2] use a definition of $\eta$ which is signature dependent. In our convention and the one of [25], $\tau=-\eta$ is signature independent, and all signature dependent factors in the relations $(2.3)$ are explicit factors $(-1)^{t}$. This is natural, because, as we will see later, $\tau$ and another parameter $\sigma$ are invariants which characterize the properties of a complex bilinear form with Gram matrix $C$, for which signature does not have an invariant meaning.

${ }^{16}$ In general we use $\mu=1, \ldots, D$, but for signature $(1, D-1)$ we may shift this to the conventional range. We also shift the index range when performing a dimensional reduction, see appendix $\mathrm{F}$.

${ }^{17} \sigma$ is related to the parameter $\epsilon$ used in [25] by $\sigma=-\epsilon$.
} 
With our choice (2.3) for the chirality matrix $\gamma_{*}$, this implies

$$
C_{ \pm} \gamma_{*}= \begin{cases} \pm i C_{\mp}, & \text { for } \mathrm{D}=2,6,10, \ldots \\ C_{\mp}, & \text { for } \mathrm{D}=4,8,12, \ldots\end{cases}
$$

see appendix A.4 for a collection of useful relations.

Given a choice of $A$ and $C$, we can take $B:=\left(C A^{-1}\right)^{T}$. The matrix $B$ satisfies

$$
B B^{\dagger}=\mathbb{1}, \quad B B^{*}=\epsilon \mathbb{1},
$$

where $\epsilon= \pm 1$, depending on signature. $B$ is not completely fixed by our choice of $A, C$ since $\alpha B$, where $\alpha$ is a phase factor, has the same properties. The matrix $B$ can be used to define the (family of) complex-antilinear maps

$$
J: \psi \mapsto \alpha^{*} B^{*} \psi^{*}
$$

Since

$$
J^{2}(\psi)=J\left(\alpha^{*} B^{*} \psi^{*}\right)=\alpha^{*} B^{*} \alpha B \psi=\epsilon \psi,
$$

the matrix $B$ either defines a real structure $(\epsilon=1)$ or a quaternionic structure $(\epsilon=-1)$ on $\mathbb{S}$, which is $\operatorname{Spin}(t, s)$-invariant. $\mathbb{S}$ carries a natural spin invariant complex structure

$$
I: \psi \mapsto i \psi
$$

Since $J$ is complex antilinear, $I$ and $J$ anticommute with each other and with their product $K:=I J$. Since in addition

$$
I^{2}=-\mathrm{Id}, \quad J^{2}=K^{2}=\epsilon \mathrm{Id},
$$

$I, J$ generate a real four-dimensional algebra, which for $\epsilon=-1$ is the algebra $\mathbb{H}$ of quaternions. For $\epsilon=1$, the resulting algebra $\mathbb{H}^{\prime}$ is called the algebra of para-quaternions (or split-quaternions). The algebra $\mathbb{H}^{\prime}$ is isomorphic to the algebra of real $2 \times 2$ matrices, $\mathbb{H}^{\prime} \cong \mathbb{R}(2)$. Compared to the quaternions, the two generators $J, K$ are not complex structures (which square to minus the identity), but para-complex structures (which square to the identity and have an equal number of eigenvalues \pm 1 ). To be able to discuss both cases in parallel, we will use the terms $\epsilon$-complex and $\epsilon$-quaternionic structure, and use the notation $J^{(\epsilon)}=J^{( \pm)}$, if $\left(J^{(\epsilon)}\right)^{2}=\epsilon \mathrm{I}$ d. We will also use the notation $\mathbb{H}_{\epsilon}$, where $\mathbb{H}_{-1}=\mathbb{H}$ and $\mathbb{H}_{1}=\mathbb{H}^{\prime}$.

The algebra $\mathbb{H}_{\epsilon}$ is a subalgebra of the Schur algebra $\mathcal{C}(\mathbb{S})$ of the complex spinor module. By comparison to the classification of Clifford algebras one can verify that this is in fact the full Schur algebra in odd dimensions. In even dimensions one has two charge conjugation matrices $C_{ \pm}$which can be used to define two $B$-matrices $B_{ \pm}$, which satisfy $B_{ \pm} B_{ \pm}^{*}=\epsilon_{ \pm} \mathrm{Id}$. The corresponding $\epsilon$-quaternionic structures are denoted $J_{ \pm}^{\left(\epsilon_{ \pm}\right)}$, where the lower index encodes the corresponding $B$-matrix $B_{ \pm}$, while the upper index encodes whether the $\epsilon_{ \pm}$-quaternionic structure is a real or quaternionic structure.

The signs $\left(\epsilon_{+}, \epsilon_{-}\right)$depend on the signature and are listed in table 1 , which again is periodic modulo 8 in dimension. Note that all three inequivalent combinations occur, that is, 
in even dimensions $\mathbb{S}$ either carries two spin invariant real structures, or two spin invariant quaternionic structures, or one structure of each type. The algebras generated by these structures are $2 \mathbb{H}^{\prime}=\mathbb{H}^{\prime} \oplus \mathbb{H}^{\prime}$ for $\left(\epsilon_{+}, \epsilon_{-}\right)=(1,1)$ (two real structures), $2 \mathbb{H}=\mathbb{H} \oplus \mathbb{H}$ for $\left(\epsilon_{+}, \epsilon_{-}\right)=(-1,-1)$ (two quaternionic structures), and the algebra $\mathbb{C}(2)$ of complex $2 \times 2$ matrices for $\left(\epsilon_{+}, \epsilon_{-}\right)=( \pm 1, \mp 1)$ (one real and one quaternionic structure). Note that in the third case $\mathbb{H} \subset \mathbb{C}(2)$ and $\mathbb{H}^{\prime} \subset \mathbb{C}(2)$, but $\mathbb{C}(2) \neq \mathbb{H} \oplus \mathbb{H}^{\prime}$. By comparison to the classification of Clifford algebras one can verify that this is the full Schur algebra $\mathcal{C}(\mathbb{S})$ in even dimensions. We refer to [17] for more details. Note that the entries in table 1 allow one to read off the Schur algebras $\mathcal{C}(\mathbb{S})=\mathcal{C}_{t, s}(\mathbb{S})$ for all signatures $(t, s)$.

In signatures where there exists a $B$-matrix which defines a real structure one can impose the reality condition

$$
\psi^{*}=\alpha B \psi
$$

on a Dirac spinor. We will refer to such spinors as Majorana spinors. Note that we do not require that the $\gamma$-matrices have real entries, and that we do not distinguish between Majorana spinors and pseudo-Majorana spinors. ${ }^{18}$

Note that Majorana spinors are not the same as real spinors, that is elements of the real spinor module $S$. Depending on signature, either $\mathbb{S} \cong S$, and real spinors are Dirac spinors, or $\mathbb{S} \cong S \otimes_{\mathbb{R}} \mathbb{C}$, and real spinors are Majorana spinors. We remark that if $\mathbb{S} \cong S$, Majorana spinors may still exist. In this case real spinors are reducible, $S \cong S_{+} \oplus S_{-}$, and Majorana spinors correspond real semi-spinors. Note that in contrast to complex semi-spinors, real semi-spinors can exist in odd dimensions. The interested reader is referred to appendix B for details.

\subsection{Complexification of spinor modules}

The odd part $\mathfrak{g}_{1}$ of a Poincaré Lie superalgebra consists of copies of irreducible real spinor representations. If the real spinor module $S$ is the unique irreducible real spinor representation, then the only choice we have is the number $N$ of copies, $\mathfrak{g}_{1}=S^{\oplus N} \cong S \otimes \mathbb{R}^{N}$. If $S$ is reducible, but the real semi-spinor modules are isomorphic, $S_{+} \cong S_{-}$, we can take $\mathfrak{g}_{1}=S_{+}^{\oplus N} \cong S_{+} \otimes \mathbb{R}^{N}$ without loss of generality. If the real semi-spinors are not equivalent $S_{+} \nsucceq S_{-}$, we can choose their multiplicity independently, $\mathfrak{g}_{1}=S_{+}^{\oplus N_{+}} \oplus S_{-}^{\oplus N_{-}} \cong$ $S_{+} \otimes \mathbb{R}^{N_{+}} \oplus S_{-} \otimes \mathbb{R}^{N_{-}}$.

The complexification $\mathfrak{g}_{1}^{\mathbb{C}} \otimes_{\mathbb{R}} \mathbb{C}$ of $\mathfrak{g}_{1}$ takes the form $\mathbb{S} \otimes \mathbb{C}^{K}$ or $\mathbb{S}_{+} \otimes \mathbb{C}^{K_{+}} \oplus \mathbb{S}_{-} \otimes \mathbb{C}^{K_{-}}$, where the relation between $K, K_{+}, K_{-}$and $N, N_{+}, N_{-}$depends on whether $\mathbb{S}, \mathbb{S}_{ \pm}$carry a $\operatorname{Spin}(t, s)$ invariant real structure, or not. Here we summarize these relations, while details are given in appendix $\mathrm{C}$. There are three cases, depending on the properties of the real spinor module $S$ :

1. $S$ irreducible:

$$
\mathfrak{g}_{1} \otimes_{\mathbb{R}} \mathbb{C}=S^{\oplus N} \otimes_{\mathbb{R}} \mathbb{C}=\mathbb{S} \otimes_{\mathbb{C}} \mathbb{C}^{K},\left\{\begin{array}{l}
K=N, \text { if } \mathbb{S} \cong S \otimes_{\mathbb{R}} \mathbb{C} \\
K=2 N, \text { if } \mathbb{S} \cong S
\end{array}\right.
$$

\footnotetext{
${ }^{18}$ This distinction may be relevant for deciding which type of terms, for example mass terms, can appear in a supersymmetry Langrangian. This is beyond the scope of our paper, see for example [2] for a discussion.
} 


\begin{tabular}{|c|c|c|c|c|c|c|c|}
\hline$D$ & $(0, D)$ & $(1, D-1)$ & $(2, D-2)$ & $(3, D-3)$ & $(4, D-4)$ & $(5, D-5)$ & $(6, D-6)$ \\
\hline 1 & +1 & +1 & & & & & \\
\hline 2 & $-1_{+},+1_{-}$ & $+1_{+}+1_{-}$ & $+1_{+},-1_{-}$ & & & & \\
\hline 3 & -1 & +1 & +1 & -1 & & & \\
\hline 4 & $-1_{+},-1_{-}$ & $+1_{+},-1_{-}$ & $+1_{+},+1_{-}$ & $-1_{+},+1_{-}$ & $-1_{+},-1_{-}$ & & \\
\hline 5 & -1 & -1 & +1 & +1 & -1 & -1 & \\
\hline 6 & $+1_{+},-1_{-}$ & $-1_{+},-1_{-}$ & $-1_{+},+1_{-}$ & $+1_{+},+1_{-}$ & $+1_{+},-1_{-}$ & $-1_{+},-1_{-}$ & $-1_{+},+1_{-}$ \\
\hline 7 & +1 & -1 & -1 & +1 & +1 & -1 & -1 \\
\hline 8 & $+1_{+},+1_{-}$ & $-1_{+},+1_{-}$ & $-1_{+},-1_{-}$ & $+1_{+},-1_{-}$ & $+1_{+},+1_{-}$ & $-1_{+},+1_{-}$ & $-1_{+},-1_{-}$ \\
\hline 9 & +1 & +1 & -1 & -1 & +1 & +1 & -1 \\
\hline 10 & $-1_{+},+1_{-}$ & $+1_{+},+1_{-}$ & $+1_{+},-1_{-}$ & $-1_{+},-1_{-}$ & $-1_{+},+1_{-}$ & $+1_{+},+1_{-}$ & $+1_{+},-1_{-}$ \\
\hline 11 & -1 & +1 & +1 & -1 & -1 & +1 & +1 \\
\hline 12 & $-1_{+},-1_{-}$ & $+1_{+},-1_{-}$ & $+1_{+},+1_{-}$ & $-1_{+},+1_{-}$ & $-1_{+},-1_{-}$ & $+1_{+},-1_{-}$ & $+1_{+},+1_{-}$ \\
\hline
\end{tabular}

Table 1. This table lists the spin invariant real and quaternionic structures on the complex spinor module $\mathbb{S}$ for all signatures $(t, s)$ in dimensions up to twelve. Note that the table is periodic modulo 8 in dimension and therefore covers all possible dimensions and signatures. It is also invariant under $(t, s) \leftrightarrow(s, t)$, provided that in even dimension one exchanges $J_{ \pm}^{(\epsilon)}$ with $J_{\mp}^{(\epsilon)}$. The entries in the table are the values of $\epsilon= \pm 1$ which tell us whether the $\epsilon$-quaternionic structure $J^{(\epsilon)}$ is a real structure, $\epsilon=1$, or a quaternionic structure, $\epsilon=-1$. In even dimensions the sign subscript on $\pm 1_{ \pm}$indicates whether the corresponding $\epsilon$-quaternionic structure $J_{ \pm}^{(\epsilon)}$ has been constructed using $B_{+}$or $B_{-}$. Majorana spinors exists in signatures where at least one of the entries is +1 , while symplectic Majorana spinors exist whenever at least one entry is -1 . In even dimensions, if both entries are equal to each other, reality conditions are 'Weyl-compatible,' that is they respect chirality, whereas if they are different from each other, reality conditions are 'Weyl-incompatible', that is, they flip chirality. Majorana-Weyl spinors exist when both entries are equal to +1 . If both entries are equal to -1 one has a quaternionic structure compatible with chirality and can define 'symplectic Majorana-Weyl spinors.' If the signs are not equal, one has one real and one quaternionic structures, which both flip chirality. The Schur algebra of the complex spinor module $\mathbb{S}$ is $\mathbb{H}_{\epsilon}$ in odd dimensions and either $\mathbb{H}_{\epsilon} \oplus \mathbb{H}_{\epsilon}$ (for $\epsilon_{+}=\epsilon_{-}$) or $\mathbb{C}(2)$ (for $\epsilon_{+}=-\epsilon_{-}$) in even dimensions. Some of the above statements will only be proved in later sections.

2. $S=S_{+}+S_{-}$and $S_{+} \cong S_{-}$:

$$
\mathfrak{g}_{1} \otimes_{\mathbb{R}} \mathbb{C}=S_{+}^{\oplus N} \otimes_{\mathbb{R}} \mathbb{C}=\mathbb{S} \otimes_{\mathbb{C}} \mathbb{C}^{K},
$$

where $K=N$, since this only occurs when $\mathbb{S} \cong S_{ \pm} \otimes_{\mathbb{R}} \mathbb{C}$.

3. $S=S_{+}+S_{-}$and $S_{+} \cong S_{-}$:

$$
\begin{aligned}
\mathfrak{g}_{1} \otimes_{\mathbb{R}} \mathbb{C} & =\left(S_{+}^{\oplus N_{+}} \oplus S_{-}^{\oplus N_{-}}\right) \otimes_{\mathbb{R}} \mathbb{C} \\
& =\mathbb{S}_{+} \otimes_{\mathbb{C}} \mathbb{C}^{K_{+}} \oplus \mathbb{S}_{-} \otimes_{\mathbb{C}} \mathbb{C}^{K_{-}},\left\{\begin{array}{l}
K_{ \pm}=N_{ \pm}, \text {if } \mathbb{S}_{ \pm} \cong S_{ \pm} \otimes_{\mathbb{R}} \mathbb{C}, \\
K_{ \pm}=2 N_{ \pm}, \text {if } \mathbb{S}_{ \pm} \cong S_{ \pm} .
\end{array}\right.
\end{aligned}
$$

Note that the relation between $K, K_{ \pm}$and $N, N_{ \pm}$is completely determined by the information whether $\mathbb{S}, \mathbb{S}_{ \pm}$admit invariant real structures or not. We remark that $N, N_{ \pm}$ 
do not count supersymmetries in multiples of the minimal supersymmetry algebra in a given signature. The reason is that the definition of a supersymmetry algebra requires the existence of a non-degenerate bracket $\mathfrak{g}_{1} \times \mathfrak{g}_{1} \rightarrow V$, which is not guaranteed. The convention that we find convenient for labelling supersymmetry algebras in arbitrary signature is that $\mathcal{N}=K$ in cases 1 and 2 and $\mathcal{N}_{ \pm}=K_{ \pm}$in case 3 . This means that we count in units of Majorana and Majorana-Weyl spinors, irrespective of whether these exist in the given signature. Thus in the non-chiral case the smallest supersymmetry algebra is labeled $\mathcal{N}=1$ if the supercharges form a single Majorana spinors and $\mathcal{N}=2$ if they form a Dirac spinor. In the chiral case algebras based on a single Majorana-Weyl spinor are denoted $(1,0)$ and $(0,1)$ while algebras based on a single Weyl spinor are denoted $(2,0)$ or $(0,2)$. We will illustrate how our conventions compare to standard $(1, D-1)$ signature conventions using explicit examples later, see in particular section 6.2.

\section{Bilinear forms and complex supersymmetry algebras}

The first step in our programme is to construct complex supersymmetry algebras with odd part $\mathfrak{g}_{1}^{\mathbb{C}}$ equal to

$$
\mathbb{S} \otimes \mathbb{C}^{K} \quad \text { or } \quad \mathbb{S}_{+} \otimes \mathbb{C}^{K_{+}} \oplus \mathbb{S}_{-} \otimes \mathbb{C}^{K_{-}}
$$

This requires us to specify a complex superbracket, $\mathfrak{g}_{1}^{\mathbb{C}} \times \mathfrak{g}_{1}^{\mathbb{C}} \rightarrow V^{\mathbb{C}}$, where $V^{\mathbb{C}}=\mathbb{C}^{t+s} \cong$ $\mathbb{R}^{t, s} \otimes_{\mathbb{R}} \mathbb{C}$. As explained in the introduction, this is equivalent to defining a complex symmetric, spin-equivariant vector-valued bilinear form $\Pi_{\beta}$ on $\mathfrak{g}_{1}^{\mathbb{C}}$, which in turn can be defined using a super-admissible complex bilinear form $\beta$ on $\mathfrak{g}_{1}^{\mathbb{C}}$. In this section we show how such forms are constructed out of bilinear forms on the complex spinor modules $\mathbb{S}$ and $\mathbb{S}_{ \pm}$, and on the auxiliary spaces $\mathbb{C}^{K}$ and $\mathbb{C}^{K_{ \pm}}$.

\subsection{Bilinear forms on the complex spinor module $\mathbb{S}$}

The definitions of symmetry $\sigma_{\beta}$ and type $\tau_{\beta}$ of a bilinear form $\beta$ were given (1.8). Bilinear forms of definite type and symmetry are called admissible and are automatically spin invariant. The choice of an admissible complex bilinear form on $\mathbb{S}$ is equivalent to the choice of a charge conjugation matrix $C$,

$$
C: \mathbb{S} \times \mathbb{S} \rightarrow \mathbb{C}, C(\lambda, \chi)=\lambda^{T} C \chi=\lambda_{\alpha} C^{\alpha \beta} \chi_{\beta} .
$$

We will refer to such bilinear forms as Majorana bilinear forms. The symmetry $\sigma_{C}$ and type $\tau_{C}$ of the bilinear form $C$ are equal to the parameters $\sigma, \tau$ associated with a charge conjugation matrix, $\sigma=\sigma_{C}, \tau=\tau_{C}$. The values of $\sigma, \tau$ are listed in table 2 .

Given an admissible bilinear form on $\mathbb{S}$, we can define a spin equivariant vector valued form

$$
\beta^{(1)}: \mathbb{S} \times \mathbb{S} \rightarrow V^{\mathbb{C}}, \quad(s, t) \mapsto \beta\left(\gamma^{\mu} s, t\right) e_{\mu},
$$

which is symmetric for $\sigma \tau=1$ and anti-symmetric for $\sigma \tau=-1$. Symmetric vector valued bilinear forms on $\mathbb{S}$ define a Poincaré Lie superalgebra with $\mathfrak{g}_{1}^{\mathbb{C}}=\mathbb{S}$, and the associated scalar bilinear forms are called super-admissible. Since we are interested in defining supersymmetry 
algebras with $\mathfrak{g}_{1}^{\mathbb{C}}=\mathbb{S} \otimes \mathbb{C}^{K}$, we are not restricted to super-admissible bilinear forms, since we have the additional freedom of choosing a bilinear form on $\mathbb{C}^{K}$. Therefore all admissible bilinear forms on $\mathbb{S}$ qualify as building blocks for superbrackets.

\subsection{Bilinear forms on the Weyl spinor modules $\mathbb{S}_{ \pm}$}

In even dimensions the complex spinor module decomposes into the complex semi-spinor modules $\mathbb{S}_{ \pm}$, and we can define two inequivalent complex bilinear forms $C_{-\tau}=C_{ \pm}$using the two available charge conjugation matrices. Let us consider the case where one argument of the bilinear form $C_{-\tau}$ is a Weyl spinor, while the other is arbitrary. Using $\gamma_{*} \lambda_{ \pm}= \pm \lambda_{ \pm}$ and (A.23) we find

$$
C_{-\tau}\left(\cdot, \lambda_{ \pm}\right)=C_{-\tau}\left(\cdot, \pm \gamma_{*} \lambda_{ \pm}\right)= \begin{cases}i C_{\tau}\left(\cdot, \lambda_{ \pm}\right), & \mathrm{D}=2,6,10, \ldots \\ \pm C_{\tau}\left(\cdot, \lambda_{ \pm}\right), & \mathrm{D}=4,8,12, \ldots\end{cases}
$$

We observe that the two bilinear forms become proportional when we restrict one argument to be a Weyl spinor. This shows that the two charge conjugation matrices and bilinear forms only differ by a relative sign or factor of $i$ between their restrictions to the complex semi-spinor modules. For any admissible bilinear form $\beta$ on $\mathbb{S}=\mathbb{S}_{+} \oplus \mathbb{S}_{-}$we can define an associate admissible bilinear form $\beta^{\prime}=\beta\left(\cdot, \gamma_{*} \cdot\right)$ which has opposite type and is proportional to $\beta$ when restricted to a definite chirality in one argument.

Admissible bilinear forms on $\left(\mathbb{S}_{+} \oplus \mathbb{S}_{-}\right) \times\left(\mathbb{S}_{+} \oplus \mathbb{S}_{-}\right)$have a third invariant besides the symmetry $\sigma_{\beta}$ and the type $\tau_{\beta}$. An admissible bilinear form $\beta$ has isotropy $\iota_{\beta, 0}=1$ and is called orthogonal if its restrictions to $\mathbb{S}_{+} \times \mathbb{S}_{-}$and $\mathbb{S}_{-} \times \mathbb{S}_{+}$are identically zero, and it has isotropy $\iota_{\beta, 0}=-1$ and is called isotropic if its restrictions to $\mathbb{S}_{+} \times \mathbb{S}_{+}$and $\mathbb{S}_{-} \times \mathbb{S}_{-}$are identically zero. To determine the isotropy $\iota_{\beta, 0}$ we use that bilinear forms which differ by the insertion of $\gamma_{*}$ in one argument are proportional to one another when restricted to a fixed chirality in one argument. We compute:

$$
\begin{gathered}
C_{+}\left(\lambda_{ \pm}, \chi_{ \pm}\right)=k C_{-}\left(\lambda_{ \pm}, \chi_{ \pm}\right), \quad \text { where } k=\left\{\begin{array}{l}
i, D=2,6,10, \ldots \\
1, D=4,8,12, \ldots
\end{array}\right. \\
\Rightarrow \sigma_{+} C_{+}\left(\lambda_{ \pm}, \chi_{ \pm}\right)=k \sigma_{-} C_{-}\left(\lambda_{ \pm}, \chi_{ \pm}\right)=k k^{-1} \sigma_{-} C_{+}\left(\lambda_{ \pm}, \chi_{ \pm}\right)=\sigma_{-} C_{+}\left(\lambda_{ \pm}, \chi_{ \pm}\right),
\end{gathered}
$$

where $\sigma_{ \pm}=\sigma_{C_{ \pm}}$. Therefore either $\sigma_{+}=\sigma_{-}$, or $C_{+}$and $C_{-}$are completely degenerate when restricted to semi-spinors of the same chirality. On the other hand

$$
\begin{gathered}
C_{+}\left(\lambda_{ \pm}, \chi_{\mp}\right)=k^{\prime} C_{-}\left(\lambda_{ \pm}, \chi_{\mp}\right), \quad \text { where } k^{\prime}= \begin{cases}i, & D=2,6,10, \ldots \\
-1, & D=4,8,12, \ldots\end{cases} \\
\Rightarrow \sigma_{+} C_{+}\left(\lambda_{\mp}, \chi_{ \pm}\right)=k \sigma_{-} C_{-}\left(\lambda_{\mp}, \chi_{ \pm}\right)=k k^{\prime} \sigma_{-} C_{+}\left(\lambda_{\mp}, \chi_{ \pm}\right)=-\sigma_{-} C_{+}\left(\lambda_{\mp}, \chi_{ \pm}\right) .
\end{gathered}
$$

Note that compared to the previous case we have obtained a minus sign, because the chirality of the second argument has changed. In this case either $\sigma_{+}=-\sigma_{-}$, or the bilinear forms $C_{ \pm}$are completely degenerate on semi-spinors of opposite chirality. Thus any admissible bilinear form is either orthogonal or isotropic, and its isotropy is given by

$$
\iota_{\beta, 0}=\sigma_{+} \sigma_{-} .
$$


If we insert a $\gamma$-matrix into the first argument, this flips the isotropy, since $\gamma^{\mu}$ anti-commutes with $\gamma_{*}$ in even dimensions. More generally, if we substitute $p$-fold anti-symmetrized products of $\gamma$-matrices into $\beta$ the isotropy is given by

$$
\iota_{\beta, p}=(-1)^{p} \sigma_{+} \sigma_{-}, \quad p=0,1, \ldots .
$$

Since superbrackets are defined by vector-valued bilinear forms, the relevant isotropy is $\iota_{\beta, 1}$, which we will therefore denote by $\iota=\iota_{\beta}:=\iota_{\beta, 1}$ in the following. By inspection of table 2 , we see that vector-valued bilinear forms $C_{ \pm}\left(\gamma^{\mu} \cdot, \cdot\right)$ are orthogonal in dimension $D=2,6,10, \ldots$ and isotropic in dimension $D=4,8,12, \ldots$. This is important because 'chiral' supersymmetry algebras which only involve supersymmetry generators of one chirality (or which, more generally, have different anti-commutation relations depending on chirality) can only exist in dimensions where the vector-valued bilinear form is of orthogonal type.

\subsection{Bilinear forms on the auxiliary space $\mathbb{C}^{K}$}

To define a complex bilinear form on the extended spinor module $\mathbb{S} \otimes \mathbb{C}^{K}$, we also need to choose a bilinear form $M$ on $\mathbb{C}^{K}$,

$$
M: \mathbb{C}^{K} \times \mathbb{C}^{K} \rightarrow \mathbb{C}, \quad M(w, z)=w^{i} z^{j} M_{j i} \quad i, j=1, \ldots, M .
$$

Our index convention is chosen to be consistent with the $N W-S E$ convention in the case where $M$ is antisymmetric. Since the spin group does not act on the multiplicity space $\mathbb{C}^{K}$, spin-equivariance is not an issue. If the bilinear form $\beta$ we have chosen on $\mathbb{S}$ is super-admissible, then $M$ needs to be symmetric, if $\beta$ is not super-admissible, then $M$ needs to be antisymmetric, in order that $\beta \otimes M$ defines a superbracket on $\mathbb{S} \otimes \mathbb{C}^{K}$. The symmetry of $M$ is denoted $\sigma_{M}$,

$$
M(w, z)=\sigma_{M} M(z, w), \quad M_{i j}=\sigma_{M} M_{j i}, \quad \sigma_{M}= \pm 1 .
$$

Non-degenerate symmetric and antisymmetric complex bilinear forms on $\mathbb{C}^{K}$ are unique up to isomorphism. In the symmetric case we will use the standard symmetric bilinear form $\delta(\cdot, \cdot)$ with Gram matrix given by the Kronecker-symbol, $M_{i j}=\delta_{i j}$. In the anti-symmetric case the bilinear form is only non-degenerate for $K$ even. Since degenerate superbrackets effectively involve a smaller spinor module $\mathfrak{g}_{1}^{\mathbb{C}}$ with a non-degenerate superbracket, we don't need to consider them separately. ${ }^{19}$ For $K$ even, we use the standard non-degenerate anti-symmetric bilinear form

$$
\left(J_{K}\right)_{i j}=\left(\begin{array}{cc}
0 & \mathbb{1}_{k} \\
-\mathbb{1}_{k} & 0
\end{array}\right) \quad K=2 k .
$$

\footnotetext{
${ }^{19}$ The vector space of real superbrackets contains degenerate superbrackets, which correspond to higher co-dimension orbits of the action of the Schur group. In particular, the completely degenerate bracket always forms a zero-dimensional orbit. If superbrackets exist which are degenerate, but not completely degenerate, they define smaller supersymmetry algebras with a lower number of supercharges. For example the $\mathcal{N}=1$ supersymmetry algebras in signatures $(1,3)$ and $(2,2)$ correspond to co-dimension one orbits in the space of $\mathcal{N}=2$ superbrackets, see [17]. Such real superbrackets can also be obtained directly by imposing a reality condition on a non-degenerate complex superbracket and the restriction to even $K$ does not restrict the generality of our method.
} 
$\left(J_{2}\right)_{i j}$ is the Levi-Civita symbol $\epsilon_{i j}$. The bilinear form represented by the Gram matrix $\left(J_{K}\right)_{i j}$ will be denoted $J(\cdot, \cdot)$ or just $J$. The $K$ subscript will be omitted when the context is clear.

The groups acting linearly on $\mathbb{C}^{K}$ under which these bilinear forms are invariant will be denoted $G_{\mathbb{C}^{K}}$. Depending on the symmetry of $M$ the invariance group is either the complex orthogonal group or the complex symplectic group:

$$
G_{\mathbb{C}^{K}}= \begin{cases}\mathrm{O}(K, \mathbb{C}), & \text { for } M=\delta, \\ \operatorname{Sp}(K, \mathbb{C}), & \text { for } M=J\end{cases}
$$

As we will see, the R-symmetry groups of the real supersymmetry algebras will be determined by the groups $G_{\mathbb{C}^{K}}$, together with isotropy properties of the bilinear form and the reality conditions.

\subsection{Complex supersymmetry algebras}

By combining our previous results, we can now define complex superbrackets.

\subsubsection{Odd dimensions}

In odd dimensions, the complexified spinor module is always of the form $\mathbb{S} \otimes \mathbb{C}^{K}$, and bilinear forms can be built by taking tensor products of bilinear forms on each factor. We will use the notation $\beta=C \otimes M$, where

$$
\beta:\left(\mathbb{S} \otimes \mathbb{C}^{K}\right) \times\left(\mathbb{S} \otimes \mathbb{C}^{K}\right) \rightarrow \mathbb{C}, \beta(\lambda, \chi)=\left(\lambda^{i}\right)^{T} C \chi^{j} M_{j i}
$$

It will be useful to display indices referring to the multiplicity space $\mathbb{C}^{K}$ explicitly while suppressing spinor indices.

The symmetry of $\beta$ is the product of those of $C$ and $M$, while the type is inherited from $C$ since the $\gamma$-matrices do not act on $\mathbb{C}^{K}: \sigma_{\beta}=\sigma_{C} \sigma_{M}, \tau_{\beta}=\tau_{C}$. To define a superbracket we need $\sigma_{\beta} \tau_{\beta}=\sigma_{C} \tau_{C} \sigma_{M}=1$.

\subsubsection{Even dimensions}

In even dimensions we have to distinguish two cases. If the real spinor module is irreducible then the extended spinor module has the same form $\mathbb{S} \otimes \mathbb{C}^{K}$ as in odd dimensions, and everything works like there. In signatures where the real spinor module decomposes into non-isomorphic real semi-spinor modules the extended spinor module is $\mathbb{S}_{+} \otimes \mathbb{C}^{K_{+}} \oplus \mathbb{S}_{-} \otimes$ $\mathbb{C}^{K_{-}}$. How we proceed depends on whether the vector-valued Majorana bilinear forms are orthogonal or isotropic on $\mathbb{S}=\mathbb{S}_{+} \oplus \mathbb{S}_{-}$, which is determined by $\iota=\iota_{\beta, 1}=-\sigma_{+} \sigma_{-}$. This only depends on the dimension and is unaffected by the $\mathbb{C}^{K}$ factor.

For orthogonal vector-valued bilinear forms we can have $K_{+} \neq K_{-}$, and the corresponding bilinear forms on $\mathbb{C}^{K_{ \pm}}$will be called $M_{ \pm}$. We can choose bilinear forms on each Weyl 
spinor module individually:

$$
\begin{aligned}
& \beta_{+}^{(1)}:\left(\mathbb{S}_{+} \otimes \mathbb{C}^{K_{+}}\right) \times\left(\mathbb{S}_{+} \otimes \mathbb{C}^{K_{+}}\right) \rightarrow V_{\mathbb{C}} \\
& \beta_{+}\left(\gamma^{\mu} \lambda_{+}, \chi_{+}\right)=\left(\gamma^{\mu} \lambda_{+}^{i}\right)^{T} C \chi_{+}^{j} M_{+j i} \\
& \beta_{-}^{(1)}:\left(\mathbb{S}_{-} \otimes \mathbb{C}^{K_{-}}\right) \times\left(\mathbb{S}_{-} \otimes \mathbb{C}_{-}^{K}\right) \rightarrow V_{\mathbb{C}} \\
& \beta_{-}\left(\gamma^{\mu} \lambda_{-}, \chi_{-}\right)=\left(\gamma^{\mu} \lambda_{-}^{i}\right)^{T} C \chi_{-}^{j} M_{-j i} .
\end{aligned}
$$

Note that we have suppressed an additional ' \pm ' related to the choice of the charge conjugation matrix $C_{ \pm}$for notational simplicity. Also note that without loss of generality we can choose the same Majorana bilinear form on $\mathbb{S}_{+}$and $\mathbb{S}_{-}$, since choosing different Majorana bilinear forms only changes the bilinear form on the extended spinor module $\mathbb{S}_{ \pm} \otimes \mathbb{C}^{K_{ \pm}}$by an overall factor.

For an isotropic vector-valued Majorana bilinear form we necessarily need $K_{+}=K_{-}$to define a non-degenerate bracket. The vector-valued bilinear form is

$$
\begin{aligned}
& \beta^{(1)}:\left(\mathbb{S}_{ \pm} \otimes \mathbb{C}^{K}\right) \times\left(\mathbb{S}_{\mp} \otimes \mathbb{C}^{K}\right) \rightarrow V_{\mathbb{C}} \\
& \beta\left(\gamma^{\mu} \lambda_{ \pm}, \chi_{\mp}\right)=\left(\gamma^{\mu} \lambda_{ \pm}^{i}\right)^{T} C \chi_{\mp}^{j} M_{j i} .
\end{aligned}
$$

The extended spinor module is $\mathbb{S}_{+} \otimes \mathbb{C}^{K} \oplus \mathbb{S}_{-} \otimes \mathbb{C}^{K}$, and it is natural to combine the Weyl spinors into Dirac spinors, $\lambda^{i}=\lambda_{+}^{i}+\lambda_{-}^{i}$, so that one works with $\mathbb{S} \otimes \mathbb{C}^{K}$. For even dimensions with isotropic bilinear forms $(D=4,8,12, \ldots)$ we will therefore construct superalgebras with supercharges that are elements of the $K$-extended spinor modules $\mathbb{S} \otimes \mathbb{C}^{K}$ regardless of whether the real spinor module is reducible or irreducible.

This completes our construction of complex supersymmetry algebras. The superbracket is defined by the choice of a charge conjugation matrix. This is unique in odd dimensions, while in even isotropic dimensions there are two inequivalent choices. In even orthogonal dimensions we can choose complex superbrackets independently in each chiral sector. The resulting R-symmetry groups will be determined in section 3.5, and in section 7 we will show that complex supersymmetry algebras are determined by their R-symmetry group. See table 2 for a list of superadmissible complex bilinear forms, complex supersymmetry algebras, and their R-symmetry groups.

\subsection{Complex R-symmetry groups}

The R-symmetry group is defined as the subgroup of the invariance group of the vectorvalued bilinear form (and hence of the associated superbracket) that commutes with the Lie algebra of the spin group. Equivalently, it is subgroup of the Schur group (centralizer of the spin Lie algebra), which leaves the superbracket invariant. The R-symmetry group of the complex vector-valued form bilinear form on $\mathfrak{g}_{1}^{\mathbb{C}}$,

$$
G_{R}^{\mathbb{C}}=\left\{R \in \mathcal{C}^{*}\left(\mathfrak{g}_{1}^{\mathbb{C}}\right) \mid \beta\left(\gamma^{\mu} R \cdot R \cdot\right)=\beta\left(\gamma^{\mu} \cdot, \cdot\right)\right\},
$$

is called the complex R-symmetry group. The complex R-symmetry group only depends on the dimension. There are three distinct cases: superalgebras in odd dimensions, in even orthogonal dimensions $(D=2,6,10, \ldots)$ and in even isotropic dimensions $(D=4,8,12, \ldots)$. 


\begin{tabular}{|cc|c|c|c|c|c|c|}
\hline$D$ & & $\sigma$ & $\tau$ & $\iota$ & $M$ & $G_{\mathbb{C}^{K}}$ & $G_{R}^{\mathbb{C}}$ \\
\hline 1 & & +1 & +1 & $\mathrm{~N} / \mathrm{A}$ & $\delta$ & $\mathrm{O}(K, \mathbb{C})$ & $\mathrm{O}(K, \mathbb{C})$ \\
\hline 2 & $C_{+}$ & -1 & -1 & +1 & $J$ & $\mathrm{O}(K, \mathbb{C})$ & $\mathrm{O}\left(K_{+}, \mathbb{C}\right) \times \mathrm{O}\left(K_{-}, \mathbb{C}\right)$ \\
& $C_{-}$ & +1 & +1 & +1 & $\delta$ & $\mathrm{O}(K, \mathbb{C})$ & \\
\hline 3 & & -1 & -1 & $\mathrm{~N} / \mathrm{A}$ & $\delta$ & $\mathrm{O}(K, \mathbb{C})$ & $\mathrm{O}(K, \mathbb{C})$ \\
\hline 4 & $C_{+}$ & -1 & -1 & -1 & $\delta$ & $\mathrm{O}(K, \mathbb{C})$ & $\mathrm{GL}(K, \mathbb{C})$ \\
& $C_{-}$ & -1 & +1 & -1 & $J$ & $\mathrm{Sp}(K, \mathbb{C})$ & \\
\hline 5 & & -1 & +1 & $\mathrm{~N} / \mathrm{A}$ & $J$ & $\mathrm{Sp}(K, \mathbb{C})$ & $\mathrm{Sp}(K, \mathbb{C})$ \\
\hline 6 & $C_{+}$ & +1 & -1 & +1 & $J$ & $\mathrm{Sp}(K, \mathbb{C})$ & $\mathrm{Sp}\left(K_{+}, \mathbb{C}\right) \times \mathrm{Sp}\left(K_{-}, \mathbb{C}\right)$ \\
& $C_{-}$ & -1 & +1 & +1 & $J$ & $\mathrm{Sp}(K, \mathbb{C})$ & \\
\hline 7 & & +1 & -1 & $\mathrm{~N} / \mathrm{A}$ & $J$ & $\mathrm{Sp}(K, \mathbb{C})$ & $\mathrm{Sp}(K, \mathbb{C})$ \\
\hline 8 & $C_{+}$ & +1 & -1 & -1 & $J$ & $\mathrm{Sp}(K, \mathbb{C})$ & $\mathrm{GL}(K, \mathbb{C})$ \\
& $C_{-}$ & +1 & +1 & -1 & $\delta$ & $\mathrm{O}(K, \mathbb{C})$ & \\
\hline 9 & & +1 & +1 & $\mathrm{~N} / \mathrm{A}$ & $\delta$ & $\mathrm{O}(K, \mathbb{C})$ & $\mathrm{O}(K, \mathbb{C})$ \\
\hline 10 & $C_{+}$ & -1 & -1 & +1 & $J$ & $\mathrm{O}(K, \mathbb{C})$ & $\mathrm{O}\left(K_{+}, \mathbb{C}\right) \times \mathrm{O}\left(K K_{-}, \mathbb{C}\right)$ \\
& $C_{-}$ & +1 & +1 & +1 & $\delta$ & $\mathrm{O}(K, \mathbb{C})$ & \\
\hline 11 & & -1 & -1 & $\mathrm{~N} / \mathrm{A}$ & $\delta$ & $\mathrm{O}(K, \mathbb{C})$ & $\mathrm{O}(K, \mathbb{C})$ \\
\hline 12 & $C_{+}$ & -1 & -1 & -1 & $\delta$ & $\mathrm{O}(K, \mathbb{C})$ & $\mathrm{GL}(K, \mathbb{C})$ \\
& $C_{-}$ & -1 & +1 & -1 & $J$ & $\mathrm{Sp}(K, \mathbb{C})$ & \\
\hline
\end{tabular}

Table 2. This table provides a list of complex supersymmetry algebras. For each dimension we list the inequivalent Majorana bilinear forms (charge conjugation matrices) $C$ together with their invariants $\sigma$ (symmetry), $\tau$ (type), and, where applicable $\iota$ (isotropy). For each $C$ we list the corresponding choice of a bilinear form $M$ on the internal space $\mathbb{C}^{K}$ which makes $\beta=C \otimes M$ super-admissible, thus defining a complex supersymmetry algebra. $G_{\mathbb{C}^{K}}$ is the isometry group of $M$ and $G_{R}^{\mathbb{C}}$ the resulting complex R-symmetry group. The pattern in this table repeats modulo 8, and since $C_{+}$and $C_{-}$define isomorphic superbrackets, complex supersymmetry algebras are classified by their R-symmetry groups.

\subsubsection{Odd dimensions}

In odd dimensions the extended spinor module is $\mathbb{S} \otimes \mathbb{C}^{K}$, and the complex spinor module $\mathbb{S}$ is complex-irreducible. By Schur's lemma the R-symmetry coincides with the invariance group $G_{\mathbb{C}^{K}}$ of the bilinear form $M$ on $\mathbb{C}^{K}$. The complex bilinear form $M$ is symmetric (anti-symmetric) if the vector-valued Majorana bilinear form $C\left(\gamma^{\mu} \cdot, \cdot\right)$ on $\mathbb{S}$ is symmetric (anti-symmetric). In odd dimensions there is only one inequivalent charge conjugation matrix $C$, whose symmetry $\sigma=\sigma_{C}$ and type $\tau=\tau_{C}$ therefore determines the complex R-symmetry group:

$$
G_{R}^{\mathbb{C}}= \begin{cases}\mathrm{O}(K, \mathbb{C}), & D=1,3,9,11, \ldots \\ \operatorname{Sp}(K, \mathbb{C}), & D=5,7, \ldots\end{cases}
$$


We will use the following notation for R-symmetry transformations, which reflects that $R$ does not act on spinor indices (which have been suppressed):

$$
\lambda^{i} \rightarrow R_{j}^{i} \lambda^{j}, \quad i, j=1, \ldots K
$$

The corresponding R-symmetry Lie algebra element is written $r_{j}^{i}$ such that $R_{j}^{i}=\exp \left(r_{j}^{i}\right)$.

\subsubsection{Even dimensions, orthogonal bilinear form}

In even dimensions the complex spinor module is reducible, $\mathbb{S}=\mathbb{S}_{+} \oplus \mathbb{S}_{-}$, and the complex semi-spinor modules $\mathbb{S}_{ \pm}$are inequivalent as complex modules. Therefore R-symmetry transformations act block-diagonally on the associated multiplicity spaces $\mathbb{C}^{K_{ \pm}}$. Further details depend on whether the bilinear form preserves or flips chirality.

In orthogonal dimensions $D=2,6,10, \ldots$, the vector-valued bilinear form preserves chirality, and therefore the complex R-symmetry group acts independently on left- and right-handed spinors. Using the matrix notation explained in appendix D, they take the form

$$
\left(\begin{array}{l}
\underline{\lambda}_{+} \\
\underline{\lambda}_{-}
\end{array}\right) \rightarrow R\left(\begin{array}{l}
\underline{\lambda}_{+} \\
\underline{\lambda}_{-}
\end{array}\right)=\left(\begin{array}{cc}
A & 0 \\
0 & B
\end{array}\right)\left(\begin{array}{l}
\underline{\lambda}_{+} \\
\underline{\lambda}_{-}
\end{array}\right)=\left(\begin{array}{cc}
A^{i} & 0 \\
0 & B_{\hat{j}}^{\hat{i}}
\end{array}\right)\left(\begin{array}{c}
\lambda_{+}^{j} \\
\lambda_{-}^{\hat{j}}
\end{array}\right),
$$

with $i=1, \ldots, K_{+}$and $\hat{i}=1, \ldots, K_{-}$. The matrices $A^{i}{ }_{j}$ and $B_{\hat{j}}^{\hat{i}}$ act only on the internal spaces $\mathbb{C}^{K_{+}}$and $\mathbb{C}^{K_{-}}$because $\mathbb{S}_{ \pm}$are complex irreducible and by Schur's lemma R-symmetry transformations are inert on the spinor indices. ${ }^{20}$

Invariance of the vector-valued bilinear form defined by the matrices $M, M^{\prime}$ implies

$$
R^{T}\left(\begin{array}{cc}
M & 0 \\
0 & M^{\prime}
\end{array}\right) R=\left(\begin{array}{cc}
M & 0 \\
0 & M^{\prime}
\end{array}\right)
$$

which, after inserting the components of $R$, becomes

$$
\left(\begin{array}{cc}
A^{T} M A & 0 \\
0 & B^{T} M^{\prime} B
\end{array}\right)=\left(\begin{array}{cc}
M & 0 \\
0 & M^{\prime}
\end{array}\right) .
$$

By inspection of table $2 \sigma_{+} \tau_{+}=\sigma_{-} \tau_{-}$in orthogonal dimensions $D=2,6,10, \ldots$, that is, $M$ and $M^{\prime}$ are either both symmetric or both anti-symmetric, though they can have different size. Since $\sigma_{ \pm} \tau_{ \pm}=1$ for $D=2,10, \ldots$ and $\sigma_{ \pm} \tau_{ \pm}=-1$ for $D=6, \ldots$, the complex R-symmetry groups in orthogonal dimensions are:

$$
G_{R}^{\mathbb{C}}= \begin{cases}\mathrm{O}\left(K_{+}, \mathbb{C}\right) \times \mathrm{O}\left(K_{-}, \mathbb{C}\right), & D=2,10 \\ \mathrm{Sp}\left(K_{+}, \mathbb{C}\right) \times \mathrm{Sp}\left(K_{-}, \mathbb{C}\right), & D=6\end{cases}
$$

\footnotetext{
${ }^{20}$ To be precise they can act by multiplication with non-zero complex numbers, which we absorb into the action on the auxiliary space.
} 


\subsubsection{Even dimensions, isotropic bilinear forms}

In isotropic dimensions $D=4,8,12, \ldots$ R-symmetry transformations still have to act block-diagonally, but since the vector-valued bilinear form flips chirality, the blocks $A$ and $B$ must have the same size $K_{+}=K_{-}=K$,

$$
\left(\begin{array}{l}
\underline{\lambda}_{+} \\
\underline{\lambda}_{-}
\end{array}\right) \rightarrow R\left(\begin{array}{l}
\underline{\lambda}_{+} \\
\underline{\lambda}_{-}
\end{array}\right)=\left(\begin{array}{cc}
A & 0 \\
0 & B
\end{array}\right)\left(\begin{array}{l}
\underline{\lambda}_{+} \\
\underline{\lambda}_{-}
\end{array}\right)=\left(\begin{array}{cc}
A_{j}^{i} & 0 \\
0 & B_{j}^{i}
\end{array}\right)\left(\begin{array}{l}
\lambda_{+}^{j} \\
\lambda_{-}^{j}
\end{array}\right) .
$$

Invariance of the vector-valued bilinear form implies

$$
R^{T}\left(\begin{array}{cc}
0 & M \\
M & 0
\end{array}\right) R=\left(\begin{array}{cc}
0 & M \\
M & 0
\end{array}\right)
$$

This leads to

$$
\left(\begin{array}{cc}
0 & A^{T} M B \\
B^{T} M A & 0
\end{array}\right)=\left(\begin{array}{cc}
0 & M \\
M & 0
\end{array}\right)
$$

This is solved by $B=M^{-1}\left(A^{T}\right)^{-1} M$ and therefore

$$
R=\left(\begin{array}{lc}
A & 0 \\
0 & M^{-1}\left(A^{T}\right)^{-1} M
\end{array}\right) .
$$

$A$ must be invertible but is otherwise unconstrained, i.e. $A \in \mathrm{GL}(K, \mathbb{C})$. We observe

$$
\left(M^{-1}\left(A^{T}\right)^{-1} M\right)\left(M^{-1}\left(A^{\prime T}\right)^{-1} M\right)=M^{-1}\left(\left(A A^{\prime}\right)^{T}\right)^{-1} M .
$$

Therefore the complex R-symmetry group in isotropic dimensions is

$$
G_{R}^{\mathbb{C}}=\operatorname{GL}(K, \mathbb{C}), \quad D=4,8,12,
$$

and acts as the direct sum of the fundamental representation $A \rightarrow A$ with the representation $A \rightarrow M^{-1}\left(A^{T}\right)^{-1} M$ which is equivalent to the dual (contragradient) representation $A \rightarrow\left(A^{T}\right)^{-1}$.

\subsubsection{Summary table}

Our results for complex R-symmetry groups are summarized in table 2, together with information about the bilinear form $M$, the charge conjugation matrices and their invariants. The invariants were taken from [25], which uses the notation $\epsilon=-\sigma$ and $\eta=-\tau$. While the pattern repeats modulo 8 , we have included all dimensions up to 12 for convenience. In even dimensions there a two different choices $C_{ \pm}$for the charge conjugation matrix, but we will show in section 7 that there is a map which relates the superbrackets defined by $C_{ \pm}$to one another. Therefore complex supersymmetry algebras are classified by their R-symmetry groups. 


\section{Reality conditions and $\epsilon$-quaternionic structures}

So far we have constructed complex Poincaré Lie superalgebras. To obtain real supersymmetry algebras we need to impose reality conditions, which must be $\operatorname{Spin}(t, s)$ equivariant and compatible with the superbracket. In this section we deal with the first condition, while the second condition will be the subject of the next section.

Since $\mathfrak{g}_{1}^{\mathbb{C}}$ is the product of a complex spinor or complex semi-spinor module with an auxiliary complex vector space, the natural way to obtain reality conditions is to either take the product of two real structures or of two quaternionic structures. It is therefore convenient to use the terminology of $\epsilon$-quaternionic structures introduced in section 2.2.

\section{1 $\quad \epsilon$-quaternionic structures on the complex spinor module $\mathbb{S}$}

We have seen in section 2.2 that using the matrices $A$ and $C$ we can define a new matrix $B$

$$
B=\left(C A^{-1}\right)^{T},
$$

which satisfies $B^{*} B=\epsilon \mathbb{1}$. It can be shown that

$$
B^{*} B=\sigma(-\tau)^{t}(-1)^{t(t+1) / 2} \mathbb{1},
$$

where $\sigma, \tau$ are the symmetry and type of $C$, and where $t$ is the number of timelike dimensions. Through the dependence on $t$ the type of the structure varies with the signature. While in odd dimensions $C$ and $B$ are unique up to equivalence, there are two inequivalent choices of $C$, and hence of $B$, in even dimensions, denoted $B_{-\tau}=\left(C_{-\tau} A^{-1}\right)^{T}$. Given the matrix $B$ we can define a one-parameter family of $\operatorname{Spin}(t, s)$-invariant maps

$$
J^{(\epsilon)(\alpha)}: \lambda \rightarrow \alpha^{*} B^{*} \lambda^{*}, \quad|\alpha|=1,
$$

which are complex anti-linear and satisfy $\left(J^{(\epsilon)(\alpha)}\right)^{2}=\epsilon \mathrm{Id}$. The presence of the phase $\alpha$ reflects that while we have conventionally fixed $B$ in terms of $A$ and $C$, there remains the freedom of multiplying $B$ by a phase. The freedom of choosing $\alpha$ is important if one wants to impose that expressions which are obtained by imposing a reality condition on a complex expression are real-valued, rather than just being restricted to a generic real subspace. We will use this in section 5 to insure that the vector-valued bilinear form obtained by imposing a reality condition on the complex vector-valued form is real-valued. See also $[16,17]$ for how this freedom is used when constructing supersymmetric theories. To simplify notation, we will omit the superscript $(\alpha)$ whenever the value of the phase is unimportant.

In even dimensions, we have two possible charge conjugation matrices and two corresponding $\operatorname{Spin}(t, s)$-invariant $\epsilon$-quaternionic structures

$$
J_{ \pm}^{(\epsilon)(\alpha)}: \lambda \rightarrow \alpha^{*} B_{ \pm}^{*} \lambda^{*}, \quad|\alpha|=1 .
$$

The subscript on $J_{ \pm}^{(\epsilon)(\alpha)}$ refers to $B_{ \pm}$being used to define the structure. Later, we will admit different numbers of copies of $\mathbb{S}_{ \pm}$, that is, $K_{+} \neq K_{-}$, and then the phase $\alpha$ will also acquire a subscript, $\alpha_{ \pm}$, since we can use different types of structures on each Weyl spinor module. 
The values of $\epsilon$ depend on the signature and are listed in table 1 for both odd and even dimensions up to dimension 12. In even dimensions a subscript \pm indicates whether the value \pm 1 of $\epsilon$ refers to $J_{+}$or $J_{-}$. As discussed in section 2.2, in even dimensions there can either be two real structures, or two quaternionic structures, or one of each type. We have made use of the natural $(t, s) \leftrightarrow(s, t)$ symmetry, though in even dimensions one must then also replace $J_{-\tau}^{(\epsilon)}$ with $J_{\tau}^{(\epsilon)}$. For example, if we have $J_{-}^{(-1)}$ in signature $(t, s)$, then in signature $(s, t)$ there will be a quaternionic structure $J_{+}^{(-1)}$. See appendix A.5 for details.

In even dimensions, the matrices $C_{ \pm}$and hence the matrices $B_{ \pm}$are related to each other through multiplication with the chirality operator $\gamma_{*}$, see appendix A.4 for explicit expressions which involve factors $\pm 1, \pm i$. This means that the difference between $C_{+}, B_{+}$ and $C_{-}, B_{-}$lies in how they act on the complex semi-spinor modules $\mathbb{S}_{ \pm}$. We need to investigate this further in order to fully understand reality conditions on extended spinor modules of the form $\mathbb{S}_{+} \otimes \mathbb{C}^{K_{+}} \oplus \mathbb{S}_{-} \otimes \mathbb{C}^{K_{-}}$. We start from the observation that the matrices $B_{ \pm}$either both commute or both anti-commute with $\gamma_{*}$, depending on the signature. Combining (A.25) and (A.26) from appendix A.4, we find

$$
B_{ \pm} \gamma_{*}= \begin{cases}(-1)^{t+1} \gamma_{*} B_{ \pm}, & D=2,6,10, \ldots \\ (-1)^{t} \gamma_{*} B_{ \pm}, & D=4,8,12, \ldots\end{cases}
$$

Since $\gamma_{*}$ is real, the $\epsilon$-quaternionic structure $J_{ \pm}^{(\epsilon)}$ (anti-)commutes with $\gamma_{*}$ if and only if $B_{ \pm}$ does. Therefore $J_{ \pm}^{(\epsilon)}$ either preserves chirality and restricts to an $\epsilon$-quaternionic structures on the semi-spinor modules $\mathbb{S}_{ \pm}$, or it flips chirality and maps the two semi-spinor modules to one another

$$
J_{ \pm}^{(\epsilon)}: \mathbb{S}_{ \pm} \rightarrow \mathbb{S}_{ \pm}, \text {or } \quad J_{ \pm}^{(\epsilon)}: \mathbb{S}_{ \pm} \rightarrow \mathbb{S}_{\mp} .
$$

We will refer to $\epsilon$-quaternionic structures which preserve chirality as Weyl-compatible and to those which flip chirality as Weyl-incompatible. Since Weyl compatibility only depends on the space-time signature, we will also use the terminology of Weyl-compatible signatures and Weyl-incompatible signatures.

The Weyl (in-)compatibility of $J_{ \pm}^{(\epsilon)}$ is correlated with $J_{+}^{(\epsilon)}$ and $J_{-}^{(\epsilon)}$ being of the same or of the opposite type. Using (A.26) and (A.27) from appendix A.4 we obtain

$$
B_{+}^{*} B_{+}=\left\{\begin{array}{lll}
i B_{-}^{*} \gamma_{*} B_{+}, & =(-1)^{t+1} B_{-}^{*} B_{-} & D=2,6,10 \\
B_{-}^{*} \gamma_{*} B_{+}, & =(-1)^{t} B_{-}^{*} B_{-} & D=4,8,12 .
\end{array}\right.
$$

which by comparison to (4.5) shows that two $\epsilon$-complex structures $J_{ \pm}^{(\epsilon)}$ are either of the same type and both Weyl-compatible, or of opposite type and both Weyl-incompatible. These properties alternate with signature, which is due to the fact that the increase of timelike directions adds one $\gamma$-matrix to the matrix $A$, and hence to $B_{ \pm}$. The pattern is shifted between the orthogonal dimensions $D=2,6,10$ and the isotropic dimensions $D=4,8,12$. The values of $\epsilon$ for $J_{ \pm}^{(\epsilon)}$ have been listed in table 1 .

We also note that in Weyl-compatible signatures the Schur algebra of $\mathbb{S}$ is semi-simple, while in Weyl-incompatible signatures it is simple. This reflects that $C_{ \pm}$and, hence, $B_{ \pm}$ and $J_{ \pm}^{(\epsilon)}$ are related through multiplication by $\gamma_{*}$. In the case of semi-simple Schur algebra 
$\mathbb{H}_{\epsilon} \oplus \mathbb{H}_{\epsilon}$, the $\epsilon$-quaternionic structures in each factor are of the same type which leads to a Weyl-compatible action of the Schur group which respects the decomposition into semi-spinor modules. Indeed, using (A.27) we can see explicitly that the Weyl-compatible $\epsilon$-complex structures $J_{+}^{(\epsilon)}$ and $J_{-}^{(\epsilon)}$ become proportional when restricted to Weyl spinor modules

$$
J_{+}^{(\epsilon)(\alpha)}\left(\lambda_{ \pm}\right)= \begin{cases}\alpha^{*} B_{+}^{*} \lambda_{ \pm}^{*}=i \alpha^{*} B_{-}^{*} \lambda_{ \pm}^{*}=J_{-}^{(\epsilon)(i \alpha)}\left(\lambda_{ \pm}\right), & D=2,6,10 \\ \alpha^{*} B_{+}^{*} \lambda_{\mp}^{*}=\mp \alpha^{*} B_{-}^{*} \lambda_{ \pm}^{*}=J_{-}^{(\epsilon)(\mp \alpha)}\left(\lambda_{ \pm}\right), & D=4,8,12\end{cases}
$$

If the Schur algebra is the simple algebra $\mathbb{C}(2)$, there are two $\epsilon$-quaternionic structures of opposite type, which are related by the generator $\gamma_{*}$, but this time they map the Weyl spinor modules to each other.

Let us note the implications for the construction of supersymmetry algebras, in particular for the existence of chiral supersymmetry algebras where supersymmetry acts differently on left-handed and right-handed supercharges, including the option to have a different number of left- and right-handed supercharges. In Weyl-compatible signatures the Weyl spinor modules carry an $\epsilon$-quaternionic structure and are therefore self-conjugate, i.e $\overline{\mathbb{S}}_{ \pm}=\mathbb{S}_{ \pm}$. Since the Schur algebra is $\mathbb{H}_{\epsilon} \oplus \mathbb{H}_{\epsilon}$, it follows that the real spinor module is reducible $S=S_{+}+S_{-}$, and that the real semi-spinor modules are inequivalent $S_{+} \neq S_{-}$. Therefore the extended spinor module is of the form $\mathbb{S}_{+} \otimes \mathbb{C}^{K_{+}} \oplus \mathbb{S}_{-} \otimes \mathbb{C}^{K_{-}}$. To be able to define chiral real supersymmetry algebras, we also need to be in an orthogonal dimension where the complex bilinear form can be restricted to a fixed chirality $\mathbb{S}_{ \pm} \otimes \mathbb{C}^{K_{ \pm}}$, as discussed in section 3.2. Thus chiral supersymmetry algebras can be constructed when the dimension is orthogonal $(D=2,6,10, \ldots)$ and the signature is Weyl-compatible, $\epsilon_{+}=\epsilon_{-}$, where $\epsilon_{ \pm}$ is the type of $J_{ \pm}$. Isotropic bilinear forms require an equal number of spinors of both chiralities, and these can naturally be combined into Dirac spinors. In this case we will require, even in Weyl compatible signatures, that the same reality condition is imposed on both chiralities, so that supercharges and fermions can be combined into Dirac spinors. The extended spinor module is then $\mathbb{S} \otimes \mathbb{C}^{K}$. In Weyl incompatible signatures the real spinor module is either irreducible, or it is reducible and the real semi-spinors are equivalent, since in this case the Schur algebra of $S$ is $\mathbb{R}(2) \subset \mathbb{C}(2)$. Therefore the extended spinor module takes again the form $\mathbb{S} \otimes \mathbb{C}^{K}$.

\section{2 $\epsilon$-quaternionic structures on the auxiliary space $\mathbb{C}^{K}$}

Next, we need to define $\epsilon$-quaternionic structures on $\mathbb{C}^{K}$. Given a complex $K \times K$ matrix $L=\left(L_{i j}\right)$ which satisfies $L^{*} L=\epsilon \mathbb{1}$, we can define an $\epsilon$-quaternionic structure

$$
j^{(\epsilon)}: z^{i} \rightarrow\left(z^{j}\right)^{*} L_{j i}
$$

where the indices $i, j, \ldots$ comply with the NW-SE convention. Spin invariance is trivially realized, since the spin group does not act on $\mathbb{C}^{K}$. Since we want to pick a real form of a complex supersymmetry algebra, we choose reality conditions on $\mathbb{C}^{K}$ such that it picks a real form of the isometry group $G_{\mathbb{C}^{K}}$ of the complex bilinear form $M$. We will see later that this has the effect of reducing the complex R-symmetry group $G_{R}^{\mathbb{C}}$ to one of its real forms. 
Let $x \in \mathfrak{g}_{\mathbb{C}}$ be an element of the Lie algebra of the group $G_{\mathbb{C}^{K}}$ which acts on $\mathbb{C}^{K}$ in the fundamental representation. The complex linear action $z \rightarrow L z$ of $L$ on $\mathbb{C}^{K}$ defines an involution

$$
\phi_{L}: x \rightarrow L x L^{-1}, \phi_{L}^{2}=\mathbb{1}
$$

of $\mathfrak{g}_{\mathbb{C}}$, provided that we require that $L$ is real, $L=L^{*}$, so that $L^{*} L=\epsilon \mathbb{1}$ implies $L^{2}=\epsilon \mathbb{1}$ and $\phi_{L}^{2}=\mathbb{1}$. We require in addition that $\phi_{L}$ is compatible with the Lie algebra structure, that is an involutive Lie algebra automorphism. Given an involutive automorphism (4.10), we can define a real structure

$$
\tau_{L}: x \rightarrow L \bar{x} L^{-1}
$$

on the complex Lie algebra $\mathfrak{g}_{\mathbb{C}}$. The real points (fixed points) $\left(\mathfrak{g}_{\mathbb{C}}\right)^{\phi_{L}}$ of the action of $\phi_{L}$ define a real Lie algebra $\mathfrak{g}$ with complexification $\mathfrak{g}_{\mathbb{C}}$, called a real form of $\mathfrak{g}_{\mathbb{C}}$. It is a standard result that all real forms of a complex semisimple Lie algebra arise from involutive automorphisms. ${ }^{21}$ Moreover, two involutive automorphisms define the same real form if they are related through conjugation by an automorphism of $\mathfrak{g}_{\mathbb{C}}$.

We have two cases to consider.

1. The bilinear form $M$ on $\mathbb{C}^{K}$ is symmetric, with standard choice $M=\delta$. Then the Lie algebra of infinitesimal isometries is $\mathfrak{o}(K, \mathbb{C})$, and up to conjugation all involutive automorphisms are given by the following choices for $L$ :

$$
\mathbb{1}_{K}, \quad I_{p, q}=\left(\begin{array}{cc}
\mathbb{1}_{p} & 0 \\
0 & -\mathbb{1}_{q}
\end{array}\right), \quad J_{K}=\left(\begin{array}{cc}
0 & \mathbb{1}_{k} \\
-\mathbb{1}_{k} & 0
\end{array}\right), \quad K=p+q=2 k .
$$

$L=\mathbb{1}_{K}, I_{p, q}$ define a real structure $j^{(\epsilon)}=j^{(+1)}$, while $L=J_{K}$, where $K$ must be even, defines a quaternionic structure $j^{(\epsilon)}=j^{(-1)}$.

2. The bilinear form $M$ is antisymmetric, with standard choice $M=J$, and the Lie algebra of infinitesimal isometries is $\mathfrak{s p}(K, \mathbb{C})$. In this case $K=2 k$ is necessarily even, and we have the following inequivalent possibilities for $L$ :

$$
\begin{aligned}
& \mathbb{1}_{K}, \quad J_{K}=\left(\begin{array}{cc}
0 & \mathbb{1}_{k} \\
-\mathbb{1}_{k} & 0
\end{array}\right), \quad I_{1,1}=\left(\begin{array}{cc}
1 & 0 \\
0 & -1
\end{array}\right) \quad \text { (only when } K=2 \text { ), } \\
& \tilde{I}_{2 r, 2 s}=\left(\begin{array}{cccc}
\mathbb{1}_{r} & 0 & 0 & 0 \\
0 & -\mathbb{1}_{s} & 0 & 0 \\
0 & 0 & \mathbb{1}_{r} & 0 \\
0 & 0 & 0 & -\mathbb{1}_{s}
\end{array}\right)=\left(\begin{array}{cc}
I_{r, s} & 0 \\
0 & I_{r, s}
\end{array}\right), \quad k=r+s .
\end{aligned}
$$

Note that $\tilde{I}_{2 r, 2 s}$ cannot be used when $K=2$, where $I_{1,1}$ takes it place, and that in general conjugation with $I_{p, q}$ is not an involutive automorphism for $\mathfrak{s p}(K, \mathbb{C})$. For $L=\mathbb{1}_{K}, I_{1,1}, \tilde{I}_{2 r, 2 s}$ we obtain a real structure $j^{(\epsilon)}=j^{(+1)}$, and for $L=J_{K}$ we obtain a quaternionic structure $j^{(\epsilon)}=j^{(-1)}$.

\footnotetext{
${ }^{21}$ See for example [27] for real forms of complex semisimple Lie algebras.
} 
We will refer to the above choices of representatives for $L$ as 'standard' or 'canonical' in the following. The complex R-symmetry group $G_{R}^{\mathbb{C}}$, that is the invariance group of $\beta=C \otimes M$ agrees with $G_{\mathbb{C}}$ if the dimension of space-time is odd. In even dimensions $G_{R}^{\mathbb{C}}$ also depends on whether $\beta$ is orthogonal or isotropic. Real R-symmetry groups will be discussed in detail in section 6 . The real forms selected by the above automorphisms are listed in table 3 .

\subsection{Real structures on $\mathbb{S} \otimes \mathbb{C}^{K}$ and $\mathbb{S}_{+} \otimes \mathbb{C}^{K_{+}} \oplus \mathbb{S}_{-} \otimes \mathbb{C}^{K_{-}}$}

By combining information about $\epsilon$-quaternionic structures on $\mathbb{S}$ and $\mathbb{C}^{K}$, we obtain $\operatorname{Spin}(t, s)$ invariant real structures on $\mathbb{S} \otimes \mathbb{C}^{K}$, and, in even dimensions on $\mathbb{S}_{+} \otimes \mathbb{C}^{K_{+}} \oplus \mathbb{S}_{-} \otimes \mathbb{C}^{K_{-}}$.

\subsubsection{Odd dimensions}

We can define a real structure $\rho$ on $\mathbb{S} \otimes \mathbb{C}^{K}$ either as the product of two real or of two quaternionic structures.

$$
\rho=J^{(\epsilon)} \otimes j^{(\epsilon)}: \lambda^{i} \rightarrow \alpha B^{*}\left(\lambda^{j}\right)^{*} L_{j i} .
$$

Which option is available depends on the signature. In odd dimensions we have only one $\operatorname{Spin}(t, s)$-invariant structure at our disposition, which is either a real structure or a quaternionic structure. The restriction of any tensor-valued bilinear forms to this subspace is either real or purely imaginary (and therefore real after multiplication by $i$ ). We choose the phase $\alpha$ so that the vector-valued bilinear form is real.

When $K$ is odd, we cannot define quaternionic structures on $\mathbb{C}^{K}$ because a quaternionic structure requires an even number of complex dimensions. This does not impede defining a real structure on $\mathbb{S} \otimes \mathbb{C}^{K}$, because $K$ is only odd if $\mathbb{S}$ has a real structure, so that we need a real structure on $\mathbb{C}^{K}$ in order to impose a reality condition on extended spinors. Similarly, in cases where we only have access to a quaternionic structure on $\mathbb{S}$ the extended spinor modules are always of the form $\mathbb{S} \otimes \mathbb{C}^{2 K}$ so once again we can always define a real structure on the product space because there is no impediment to defining a quaternionic structure on $\mathbb{C}^{2 K}$. A corollary is that in signatures without a real structure on $\mathbb{S}$ we cannot have theories with an odd number of supersymmetries.

\subsubsection{Even dimensions}

In even dimensions we have two $\operatorname{Spin}(t, s)$-invariant structures for each signature. There are two cases to be distinguished. Either the two structures are Weyl-compatible, then they are of the same type and map $\mathbb{S}_{ \pm}$to $\mathbb{S}_{ \pm}$. Or they are Weyl-incompatible, then they are of opposite type and map $\mathbb{S}_{ \pm}$to $\mathbb{S}_{\mp}$.

Weyl-compatible $\epsilon$-quaternionic structures work in the same way as $\epsilon$-quaternionic structures in odd dimensions, except that we replace the Dirac spinor module with either Weyl-spinor module. Real structures can be defined on each Weyl spinor module individually:

$$
\rho_{( \pm)}\left(\lambda_{ \pm}^{i}\right)=\alpha B_{( \pm)}^{*}\left(\lambda_{ \pm}^{i}\right)^{*} L_{j i}
$$

where $B^{*} B=L^{2}=\epsilon$. The canonical choices of $L$ were listed above. The total real structure is then $\rho=\rho_{+}+\rho_{-}$. For superalgebras with both chiralities present, we can have different real structures defined on each chirality. 
Weyl-incompatible signatures link the two Weyl spinor modules. In terms of Weyl spinors, the real structure is of the form

$$
\rho\left(\lambda_{ \pm}^{i}\right)=\alpha B_{( \pm)}^{*}\left(\lambda_{\mp}^{i}\right)^{*} L_{j i}
$$

Which choice we make for $B$ makes a difference, as $B_{+}^{*} B_{+}=-B_{-}^{*} B_{-}$, and therefore the choice of $L$ depends on the choice of $B$. The reality condition can be written as a reality condition on $\mathbb{S} \otimes \mathbb{C}^{K}$ :

$$
\rho\left(\lambda^{i}\right)=\rho\left(\lambda_{+}^{i}\right)+\rho\left(\lambda_{-}^{i}\right)=\alpha B_{( \pm)}^{*}\left(\lambda_{-}^{j}\right)^{*} L_{j i}+\alpha B_{( \pm)}^{*}\left(\lambda_{+}^{j}\right)^{*} L_{j i}=\alpha B_{( \pm)}^{*}\left(\lambda^{j}\right)^{*} L_{j i}
$$

\section{$5 \quad$ Real supersymmetry algebras}

At this point we have all the elements in place that we need to define a real supersymmetry algebra.

1. The odd part $\mathfrak{g}_{1}^{\mathbb{C}}$ of the complex supersymmetry algebra takes the form $\mathbb{S} \otimes \mathbb{C}^{K}$ for odd dimensions and in even-dimensional signatures with isotropic bilinear forms or with Weyl-incompatible reality conditions, while it takes the form $\mathbb{S}_{+} \otimes \mathbb{C}^{K_{+}} \oplus \mathbb{S}_{-} \otimes \mathbb{C}^{K_{-}}$ in even-dimensional signatures with orthogonal vector-valued bilinear forms and Weyl-compatible reality conditions.

2. On $\mathfrak{g}_{1}^{\mathbb{C}}$ we have shown how to construct a super-admissible complex bilinear form $\beta$ (that is, forms with $\sigma_{\beta} \tau_{\beta}=1$ ). The associated complex vector-valued bilinear form

$$
\beta^{(1)}=\beta\left(\gamma^{\mu} \cdot, \cdot\right),: \quad \mathfrak{g}_{1}^{\mathbb{C}} \times \mathfrak{g}_{1}^{\mathbb{C}} \rightarrow V_{\mathbb{C}}
$$

is symmetric and $\operatorname{Spin}(t+s, \mathbb{C})$-equivariant and defines a complex superbracket, which gives $\mathfrak{s o}\left(V_{\mathbb{C}}\right) \oplus V_{\mathbb{C}} \oplus \mathfrak{g}_{1}^{\mathbb{C}}$ the structure of a complex Poincaré Lie superalgebra.

3. On $\mathfrak{g}_{1}^{\mathbb{C}}$ we have constructed $\operatorname{Spin}(t, s)$ invariant real structures $\rho$, which allow us to impose a spin-invariant reality condition and thus to obtain real forms $\mathfrak{g}_{1}$ of $\mathfrak{g}_{1}^{\mathbb{C}}$, that is $\operatorname{Spin}(t, s)$-modules whose complexification is $\mathfrak{g}_{1}^{\mathbb{C}}$.

The data that we have to specify in order to define a real supersymmetry algebra are the complex form $\mathfrak{g}_{1}^{\mathbb{C}}$ of its odd part, a super-admissible complex bilinear form $C \otimes M$ to define the complex superbracket, and the matrices $B$ and $L$ defining a real structure $\rho$ on $\mathfrak{g}_{1}^{\mathbb{C}}$, which then selects a real form $\mathfrak{g}_{1}=\left(\mathfrak{g}_{1}^{\mathbb{C}}\right)^{\rho}$ of $\mathfrak{g}_{1}^{\mathbb{C}}$. The final step to be taken in this section is to verify that if we restrict the complex vector-valued bilinear form $\beta^{(1)}$ to spinors satisfying the reality condition $\rho$, this defines a real, symmetric, $\operatorname{Spin}(t, s)$-equivariant vector-valued bilinear form

$$
\beta^{(1)}=\beta\left(\gamma^{\mu} \cdot, \cdot\right): \quad \mathfrak{g}_{1} \times \mathfrak{g}_{1} \rightarrow V
$$

on $\mathfrak{g}_{1}=\left(\mathfrak{g}_{1}^{\mathbb{C}}\right)^{\rho}$. The only property that we need to verify is that $\beta^{(1)}$ becomes real-valued when restricted to spinors which satisfy the reality condition, as the other properties hold by construction. 


\subsection{Reality conditions and superbrackets}

Let us start with the simpler case where $\mathfrak{g}_{1}^{\mathbb{C}}=\mathbb{S} \otimes \mathbb{C}^{K}$, equipped with the complex superadmissible bilinear form $\beta$. The real structure $\rho$ defines the real subspace $\mathfrak{g}_{1}$ by

$$
\rho\left(\lambda^{i}\right)=\lambda^{i} \Leftrightarrow\left(\lambda^{i}\right)^{*}=\alpha B \lambda^{j} L_{j i} .
$$

From section 2.3 we know that

$$
\mathfrak{g}_{1}=\left(\mathbb{S} \otimes \mathbb{C}^{K}\right)^{\rho} \cong S^{\oplus N}
$$

where $S$ is the unique irreducible real spinor module.

We need to verify that the vector-valued bilinear form becomes real-valued when restricted to $\mathfrak{g}_{1}$,

$$
\left([C \otimes M]\left(\gamma^{\mu} \lambda, \chi\right)\right)^{*}=[C \otimes M]\left(\gamma^{\mu} \lambda, \chi\right), \quad \text { for } \quad \lambda, \chi \in\left(\mathfrak{g}_{1}^{\mathbb{C}}\right)^{\rho} \cong \mathfrak{g}_{1} .
$$

At this point it is important to take into account that our reality conditions contain an arbitrary phase factor $\alpha$. We compute

$$
\begin{aligned}
\left([C \otimes M]\left(\gamma^{\mu} \lambda, \chi\right)\right)^{*} & =\left[\left(\gamma^{\mu} \lambda^{i}\right)^{T} C \chi^{j} M_{j i}\right]^{*} \\
& =\alpha^{2}\left(\gamma^{\mu} B \lambda^{k} L_{k i}\right)^{T} C^{*} B \chi^{l} L_{l j} M_{j i} \\
& =\alpha^{2} \tau_{B}(-1)^{t}\left(\gamma^{\mu} \lambda^{i}\right)^{T} B^{T} C^{*} B \chi^{j}\left(L M L^{T}\right)_{j i}
\end{aligned}
$$

Note that in even dimensions we can choose the matrix $B=B_{ \pm}$, which defines the reality condition, independently from our choice of $C=C_{ \pm}$, which defines the bilinear form. Therefore we have written $\tau_{B}$ to indicate that we refer to the sign in (2.3). To further evaluate the right hand side requires to fix the signature. However, it is clear that we can always choose $\alpha$ such that the bilinear form is real-valued, since, for all signatures, $B^{T} C^{*} B= \pm C$ and $L^{T} M L= \pm M,{ }^{22}$ so that $\alpha^{2}= \pm 1$ is sufficient to make the bilinear form real. This fixes $\alpha$ up to a sign, so that either $\alpha= \pm 1$ or $\alpha= \pm i$. The sign is conventional, since changing the overall sign of the superbracket is an isomorphism of Poincaré Lie superalgebras, see for example [17].

The second case is where $\mathfrak{g}_{1}^{\mathbb{C}}=\mathbb{S}_{+} \otimes \mathbb{C}^{K_{+}} \oplus \mathbb{S}_{-} \otimes \mathbb{C}^{K_{-}}$. Here the bilinear form and real structure are sums of two terms, $\beta=\beta_{+}+\beta_{-}$and $\rho=\rho_{+}+\rho_{-}$, respectively. According to section 2.3 the real form of $\mathfrak{g}_{1}^{\mathbb{C}}$ is

$$
\left(\mathbb{S}_{+} \otimes \mathbb{C}^{K_{+}}\right)^{\rho_{+}} \oplus\left(\mathbb{S}_{-} \otimes \mathbb{C}^{K_{-}}\right)^{\rho_{-}} \cong S_{+}^{\oplus N_{+}} \oplus S_{-}^{\oplus N_{-}},
$$

where $S_{+} \nsucceq S_{-}$are the real semi-spinor modules. Since we have two inequivalent spin representations and an orthogonal superbracket, we must require that the restrictions of the vector-valued bilinear form to $S_{+} \times S_{+}$and $S_{-} \times S_{-}$are both real valued. This fixes the phases $\alpha_{ \pm}$of $\rho_{ \pm}$up to sign. While the overall sign of the superbracket is conventional, the relative sign between $\alpha_{+}$and $\alpha_{-}$is not and distinguishes non-isomorphic supersymmetry algebras.

\footnotetext{
${ }^{22}$ Here we use the properties of the canonicial choices for $L$ specified in section 4.2 . Note that $M$ is real and either symmetric or antisymmetric.
} 
In particular, we will see in section 8.1 that this distinguishes between the type-IIA and typeIIA* algebras in ten dimensions, which both have the same (discrete) R-symmetry group.

Our convention is to fix a complex superbracket and then to determine the phase $\alpha$ by imposing that the superbracket is real-valued on fixed points of the chosen real structure. While we find this convenient, there are other conventions in the literature, where the phase $\alpha$ is fixed but arbitrary, and the reality of the superbracket is achieved by changing the bilinear form $M$ by a phase factor if necessary. This is for example done in [2]. In section 8.1 we will see in an example how the two conventions are mapped to one another.

\subsection{Real supersymmetry algebras with $\mathfrak{g}_{1}^{\mathbb{C}}=\mathbb{S} \otimes \mathbb{C}^{K}$}

In this section we take a closer look a supersymmetry algebras with $\mathfrak{g}_{1}^{\mathbb{C}} \cong \mathbb{S} \otimes \mathbb{C}^{K}$. All these algebras are defined by specifying a complex vector-valued bilinear form $\beta=C \otimes M$,

$$
\beta\left(\gamma^{\mu} \lambda, \chi\right)=\left(\gamma^{\mu} \lambda^{i}\right)^{T} C \chi^{j} \otimes M_{j i}
$$

and a reality condition

$$
\left(\lambda^{i}\right)^{*}=\alpha B \lambda^{j} L_{j i}
$$

As mentioned in the introduction and explained in detail in appendix A.3 this is equivalent to the anti-commutation relations

$$
\left\{Q_{\alpha}^{i}, Q_{\beta}^{j}\right\}=M^{i j}\left(\gamma^{\mu} C^{-1}\right)_{\alpha \beta} P_{\mu},
$$

with supercharges $Q^{i}=\left(Q_{\alpha}^{i}\right)$ that satisfy the reality condition $\rho\left(Q^{i}\right)=Q^{i}$, i.e.

$$
\left(Q^{i}\right)^{*}=\alpha B Q^{j} L_{j i}
$$

In odd dimensions the superbracket is unique, as there is a single choice for $C$ and this fixes the choice of $M$. Real supersymmetry algebras are then determined by the choice of $L$, subject to the condition that $B$ and $L$ together define a real structure. Note that if $C$ is not super-admissible, $M$ is antisymmetric so that the range of $i, j$ is necessarily even, preventing the existence of supersymmetry algebras based on a single irreducible spinor of supercharges.

In even dimensions we have to distinguish between orthogonal dimensions where the vector-valued bilinear form preserves chirality, and isotropic dimensions where chirality is flipped. In the orthogonal case the bilinear forms $C_{ \pm}$are either both super-admissible, or both are not, and therefore require the same $M$ to define a super-admissible $C_{ \pm} \otimes M$. One can write the superbracket in terms of chiral supercharges if desired,

$$
\left\{Q_{\alpha}^{i}, Q_{\beta}^{j}\right\}=\left\{Q_{+\alpha}^{i}, Q_{+\beta}^{j}\right\}+\left\{Q_{-\alpha}^{i}, Q_{-\beta}^{j}\right\},
$$

but note that we are currently considering the case where the real semi-spinor modules are equivalent.

In isotropic dimensions one Majorana bilinear form is super-admissible, and one is anti-super-admissible. Let us denote the super-admissible bilinear form $C$, and the antisuper-admissible bilinear form $C^{\prime}$ (either could be $C_{ \pm}$depending on dimension). There are 
two potentially non-equivalent superbrackets:

$$
\begin{aligned}
& \left\{Q_{\alpha}^{i}, Q_{\beta}^{j}\right\}=\delta^{i j}\left(\gamma^{\mu} C\right)_{\alpha \beta}^{-1} P_{\mu}, \\
& \left\{Q_{\alpha}^{i}, Q_{\beta}^{j}\right\}=J^{i j}\left(\gamma^{\mu} C^{\prime}\right)_{\alpha \beta}^{-1} P_{\mu} .
\end{aligned}
$$

In section 7.4 we will show that the two bilinear forms in fact define isomorphic real supersymmetry algebras if (any) Weyl-compatible reality condition is imposed. The relations between real algebras with Weyl-incompatible reality conditions will be discussed in section 7.5. If desired these algebras can be written in terms of chiral supercharges

$$
\left\{Q_{\alpha}^{i}, Q_{\beta}^{j}\right\}=\left\{Q_{+\alpha}^{i}, Q_{-\beta}^{j}\right\}+\left\{Q_{-\alpha}^{i}, Q_{+\beta}^{j}\right\}=2\left\{Q_{+\alpha}^{i}, Q_{-\beta}^{j}\right\} .
$$

\subsection{Real supersymmetry algebras with $\mathfrak{g}_{1}^{\mathbb{C}}=\mathbb{S}_{+} \otimes \mathbb{C}^{K_{+}} \oplus \mathbb{S}_{-} \otimes \mathbb{C}^{K_{-}}$}

Orthogonal Weyl-compatible signatures are special, since we can restrict the superbracket to real semi-spinor modules which are not isomorphic to each other. This allows to define chiral supersymmetry algebras where the positive and negative chirality supercharges are neither related by the superbracket nor by the reality condition and thus are completely independent.

The supersymmetry anti-commutators take the form

$$
\left\{Q_{+, \alpha}^{i}, Q_{+, \beta}^{j}\right\}=M^{i j}\left(\gamma^{\mu} C\right)_{\alpha \beta} P_{\mu}, \quad\left\{Q_{-, \alpha}^{i^{\prime}}, Q_{-, \beta}^{j^{\prime}}\right\}=M^{i^{\prime} j^{\prime}}\left(\gamma^{\mu} C^{\prime}\right)_{\alpha \beta} P_{\mu},
$$

where $i, j=1, \ldots \mathcal{N}_{+}$and $i^{\prime}, j^{\prime}=1, \ldots \mathcal{N}_{-}$, and where $C, C^{\prime}$ are the restrictions of the charge conjugation matrix to $\mathbb{S}_{ \pm}$.

If the Majorana bilinear forms on the semi-spinor modules are super-admissible, then $M^{i j}, M^{i j}$ are symmetric and we can define a supersymmetry algebra using a single Weyl spinor module. Since $\mathcal{N}_{ \pm}$counts supercharges in multiples of Majorana-Weyl spinors, the smallest chiral supersymmetry algebras have the form $\left(\mathcal{N}_{+}, \mathcal{N}_{-}\right)=(1,0),(0,1)$ if the supercharges are Majorana-Weyl spinors and $(2,0),(0,2)$ if they are Weyl spinors. If the restrictions of the Majorana bilinear form to $\mathbb{S}_{ \pm}$are not super-admissible, we need to choose an antisymmetric bilinear form on $\mathbb{C}^{K}$ which is only non-degenerate if $K$ is even. In this case the minimal chiral superalgebras involve two semi-spinor modules and take the form $(2,0),(0,2) \cdot{ }^{23}$

\section{$6 \quad$ R-symmetry groups}

So far we have shown that we can construct, in a uniform way across dimensions and signatures, real supersymmetry algebras with given odd part $\mathfrak{g}_{1}$ using the data $(C, M, B, L)$ defined on its complexification $\mathfrak{g}_{1}^{\mathbb{C}}$. These data are not independent, and, moreover, different sets may define isomorphic supersymmetry algebras. As a first step towards classification,

\footnotetext{
${ }^{23}$ Note that since in Weyl compatible signatures $\mathbb{S}_{ \pm}$carries either a real or a quaternionic structure, we can always impose a reality condition $\mathbb{S}_{ \pm} \otimes \mathbb{C} \mathbb{C}^{2}$ to define a $(2,0)$ or $(0,2)$ algebra, that is, $(4,0)$ and $(0,4)$ algebras are never minimal. In other words, whenever Majorana-Weyl spinors do not exist, we define 'symplectic Majorana-Weyl spinors'.
} 
we will construct and classify the R-symmetry groups that can occur in our construction. Supersymmetry algebras with different R-symmetry groups are clearly not isomorphic. In section 7 we will show that all supersymmetry algebras which arise from our construction are indeed classified up to isomorphism by their R-symmetry groups, together with a choice of the relative sign between $\alpha_{+}$and $\alpha_{-}$for chiral supersymmetry algebras.

\subsection{Determination of R-symmetry groups}

We now turn to the determination of the real R-symmetry groups, that is the R-symmetry groups of the real supersymmetry algebras that we obtain by imposing $\operatorname{Spin}(t, s)$-invariant reality conditions,

$$
G_{R}=\left\{R \in G_{R}^{\mathbb{C}}, \mid \rho(R \cdot)=R \rho(\cdot)\right\} .
$$

The R-symmetry groups for all signatures with dimension $\leq 12$ will be calculated in the following sections. As we now have to include reality conditions into our considerations, we have to distinguish between Weyl-compatible and Weyl-incompatible signatures. As a result there are five cases in total: odd dimensions, and the four cases in even dimensions: Weyl-compatible orthogonal, Weyl-compatible isotropic, Weyl-incompatible orthogonal and Weyl-incompatible isotropic. The results will be summarized in various tables.

\subsubsection{Odd dimensions}

In odd dimensions the extended spinor module is $\mathbb{S} \otimes \mathbb{C}^{K}$ and the complex R-symmetry group is $\mathrm{O}(K, \mathbb{C})$ or $\operatorname{Sp}(K, \mathbb{C})$. We impose a reality condition of the form

$$
\left(\lambda^{i}\right)^{*}=\alpha B \lambda^{j} L_{j i}
$$

where $B$ defines an $\epsilon$-quaternionic structure on $\mathbb{S}$ and where $\left(L_{j i}\right) \in \mathrm{GL}(K, \mathbb{C})$ is an involutive automorphism of $G_{R}^{\mathbb{C}}$.

Invariance under an R-symmetry transformation leads to

$$
\left(R_{j}^{i}\right)^{*} L_{k j}=R_{k}^{j} L_{j i} \Leftrightarrow R^{*} L^{T}=L^{T} R .
$$

All canonical choices for $L$ have a definite symmetry, i.e. $L^{T}= \pm L$, such that the above equation can be written

$$
R^{*} L=L R
$$

Lie algebra elements $r_{j}^{i}$ obey the same equation

$$
r^{*} L=L r \Longrightarrow r=L^{-1} r^{*} L .
$$

This is indeed the type of equation that defines a real form of the complex Lie algebra $\mathrm{O}(K, \mathbb{C})$ or $\operatorname{Sp}(K, \mathbb{C})$. To determine which real forms can occur as a real R-symmetry in a given signature, we need to know whether $L$ defines a real or a quaternionic structure on $\mathbb{C}^{K}$. This information is summarized in table 3. By imposing that $\epsilon$ must have the same 


\begin{tabular}{|c|c|c|c|}
\hline$G_{R}^{\mathbb{C}}$ & $G_{R}$ & $\epsilon$ & $L$ \\
\hline $\mathrm{O}(K, \mathbb{C})$ & $\mathrm{O}(K)$ & +1 & $\delta$ \\
\cline { 2 - 4 } & $\mathrm{O}(p, q)$ & +1 & $I_{p, q}$ \\
\cline { 2 - 4 } & $\mathrm{SO}^{*}(K)$ & -1 & $J_{K}$ \\
\hline \multirow{2}{*}{$\mathrm{Sp}(K, \mathbb{C})$} & $\mathrm{Sp}(K, \mathbb{R})$ & +1 & $\delta$ \\
\cline { 2 - 4 } & $\mathrm{USp}(2 r, 2 s)$ & +1 & $\tilde{I}_{2 r, 2 s}$ \\
\cline { 2 - 4 } & $\mathrm{USp}(K)$ & -1 & $J_{K}$ \\
\hline
\end{tabular}

Table 3. In this table we list the real forms of the complex Lie groups $\mathrm{O}(K, \mathbb{C})$ and $\operatorname{Sp}(K, \mathbb{C})$, which are the building blocks of the complex R-symmetry groups. Note that $p+q=K$ and $2 r+2 s=K$. The third column specifies whether the reality condition which defines the real form provides the auxiliary space $\mathbb{C}^{K}$ with a real structure $(\epsilon=1)$ or with a quaternionic structure $(\epsilon=-1)$. This decides which reality conditions are available to define a real structure on $\mathfrak{g}_{1}^{\mathbb{C}} \cong \mathbb{S} \otimes \mathbb{C}^{K}$. In the fourth column we list the standard or 'canonical' choices for the matrix $L$ which defines the reality condition.

\begin{tabular}{|c|c|c|c|c|c|c|c|}
\hline$D$ & $(0, D)$ & $(1, D-1)$ & $(2, D-2)$ & $(3, D-3)$ & $(4, D-4)$ & $(5, D-5)$ & $(6, D-6)$ \\
\hline 1 & $\mathrm{O}(p, q)$ & $\mathrm{O}(p, q)$ & & & & & \\
\hline 3 & $\mathrm{SO}^{*}(K)$ & $\mathrm{O}(p, q)$ & $\mathrm{O}(p, q)$ & $\mathrm{SO}^{*}(K)$ & & & \\
\hline 5 & $\mathrm{USp}(K)$ & $\mathrm{USp}(K)$ & $\mathrm{Sp}(K, \mathbb{R}), \mathrm{USp}(2 r, 2 s)$ & $\mathrm{Sp}(K, \mathbb{R}), \operatorname{USp}(2 r, 2 s)$ & $\mathrm{USp}(K)$ & $\mathrm{USp}(K)$ & \\
\hline 7 & $\mathrm{Sp}(K, \mathbb{R}), \mathrm{USp}(2 r, 2 s)$ & $\mathrm{USp}(K)$ & $\mathrm{USp}(K)$ & $\mathrm{Sp}(K, \mathbb{R}), \operatorname{USp}(2 r, 2 s)$ & $\mathrm{Sp}(K, \mathbb{R}), \operatorname{USp}(2 r, 2 s)$ & $\mathrm{USp}(K)$ & $\mathrm{USp}(K)$ \\
\hline 9 & $\mathrm{O}(p, q)$ & $\mathrm{O}(p, q)$ & $\mathrm{SO}^{*}(K)$ & $\mathrm{SO}^{*}(K)$ & $\mathrm{O}(p, q)$ & $\mathrm{O}(p, q)$ & $\mathrm{SO}^{*}(K)$ \\
\hline 11 & $\mathrm{SO}^{*}(K)$ & $\mathrm{O}(p, q)$ & $\mathrm{O}(p, q)$ & $\mathrm{SO}^{*}(K)$ & $\mathrm{SO}^{*}(K)$ & $\mathrm{O}(p, q)$ & $\mathrm{O}(p, q)$ \\
\hline
\end{tabular}

Table 4. This table lists the real R-symmetry groups which can occur in odd dimension with any signature, $p+q=K$.

value as for the $\epsilon$-quaternionic structure on $\mathbb{S}$, we arrive at the table 4 of real forms in odd dimensions up to $D=11$.

These two tables make manifest how the R-symmetry groups of real supersymmetry algebras vary across dimensions and signatures. Firstly, in signatures where $\mathbb{S}$ carries a real structure, in other words where Majorana spinors exist, there is a broader range of R-symmetry groups since apart from $\mathrm{O}(K)$ or $\mathrm{Sp}(K, \mathbb{R})$ there are further real forms $\mathrm{O}(p, q)$ or $\operatorname{Sp}(2 r, 2 s)$ which preserve real bilinear forms with indefinite signature. These correspond to 'twisted' Majorana conditions which introduce relative signs between the Majorana conditions imposed on different spinors. This phenomenon is known for Lorentz signature from Hull's type-* supersymmetry algebras and we will relate our formalism to the slightly different formalism used in [2] later when looking into specific examples. In signatures where $\mathbb{S}$ carries a quaternionic structure the R-symmetry group is fixed to be $\mathrm{SO}^{*}(K)$ if the Majorana bilinear form is super-admissible or $\operatorname{USp}(K)$ if it is not. The latter case corresponds, for $K=2$, to symplectic Majorana spinors. For $K=1$ the only possible group is $\mathrm{O}(1) \cong \mathbb{Z}_{2}$, which is discrete. According to our counting conventions for supersymmetries, where $\mathcal{N}=K$, so that we count in multiples of Majorana spinors, such algebras are $\mathcal{N}=1$ 


\begin{tabular}{|c|c|c|}
\hline$G_{R}^{\mathbb{C}}$ & $G_{R}$ & $\epsilon$ \\
\hline $\mathrm{O}\left(K_{+}, \mathbb{C}\right) \times \mathrm{O}\left(K_{-}, \mathbb{C}\right)$ & $\mathrm{O}\left(p_{+}, q_{+}\right) \times \mathrm{O}\left(p_{-}, q_{-}\right)$ & +1 \\
\cline { 2 - 3 } & $\operatorname{SO}^{*}\left(K_{+}\right) \times \mathrm{SO}^{*}\left(K_{-}\right)$ & -1 \\
\hline $\mathrm{Sp}\left(K_{+}, \mathbb{C}\right) \times \mathrm{Sp}\left(K_{-}, \mathbb{C}\right)$ & $\left(\operatorname{Sp}\left(K_{+}, \mathbb{R}\right)\right.$ or $\left.\mathrm{USp}\left(2 r_{+}, 2 s_{+}\right)\right) \times\left(\operatorname{Sp}\left(K_{-}, \mathbb{R}\right)\right.$ or $\left.\mathrm{USp}\left(2 r_{-}, 2 s_{-}\right)\right)$ & +1 \\
\cline { 2 - 3 } & $\mathrm{USp}\left(K_{+}\right) \times \mathrm{USp}\left(K_{-}\right)$ & -1 \\
\hline
\end{tabular}

Table 5. Here we list the real R-symmetry groups which can occur in orthogonal Weyl-compatible signatures. Note that $p_{ \pm}+q_{ \pm}=K_{ \pm}$and $2 r_{ \pm}+2 s_{ \pm}=K_{ \pm}$. The last column shows which type of $\epsilon$-quaternionic structure on $\mathbb{S}$ is correlated with a given real form of the complex R-symmetry group.

algebras. $\mathcal{N}=1$ algebras only exist in signatures where the entry in our table 4 is $\mathrm{O}(p, q)$. In all other cases the minimal value of $\mathcal{N}=K$ for a non-degenerate supersymmetry algebra is $\mathcal{N}=2$, that is, the supercharges form a Dirac spinor. For example, in five dimensions the smallest supersymmetry algebra is the $\mathcal{N}=2$ algebra, for all signatures. Note that while Majorana spinors exist in signatures $(t, s)=(2,3),(3,2)$, there is no non-degenerate superbracket which would allow to define an $\mathcal{N}=1$ supersymmetry algebra, as already observed in [16].

In 11 dimensions (and as well in 3 dimensions) the R-symmetry group alternates between $\mathrm{O}(p, q)$ and $\mathrm{SO}^{*}(K)$. The latter requires $K$ even and is related to quaternionic structures on $\mathbb{S}$ and $\mathbb{C}^{K}$. According to table 1, Majorana spinors, and, hence, $\mathcal{N}=K=1$ algebras exist in 11 dimensions for signature $(1,10),(2,9)$ and $(5,6)$. These algebras are realized in M-, M*- and M'-theory, respectively [2]. In some cases R-symmetry groups can be rewritten using accidental isomorphism between Lie groups. In particular, for $K=2$ we note that $\mathrm{SO}^{*}(2) \cong \mathrm{SO}(2), \mathrm{Sp}(2, \mathbb{R}) \cong \mathrm{SU}(1,1)$ and $\mathrm{USp}(2) \cong \mathrm{SU}(2)$.

\subsubsection{Orthogonal Weyl-compatible signatures}

In orthogonal Weyl-compatible signatures we can define superbrackets independently for the two Weyl spinor modules, and for each of them the situation is the same as for the spinor module in odd dimensions. The complex R-symmetry groups were found to be

$$
G_{R}^{\mathbb{C}}= \begin{cases}\mathrm{O}\left(K_{+}, \mathbb{C}\right) \times \mathrm{O}\left(K_{-}, \mathbb{C}\right) & D=2,10, \\ \mathrm{Sp}\left(K_{+}, \mathbb{C}\right) \times \mathrm{Sp}\left(K_{-}, \mathbb{C}\right) & D=6 .\end{cases}
$$

We can also impose reality conditions independently for each chirality. But since Weylcompatibility implies that $J_{+}^{(\epsilon)}$ and $J_{-}^{(\epsilon)}$ are either both real structures or both quaternionic structures, the allowed combinations are quite restricted. In table 5 we have listed the possible R-symmetry groups for orthogonal Weyl-compatible signatures.

\subsubsection{Orthogonal Weyl-incompatible signatures}

For orthogonal Weyl-incompatible signatures the complex vector-valued bilinear form preserves chirality while the reality condition flips it. Therefore the extended spinor module is of the form $\mathbb{S} \otimes \mathbb{C}^{K}$, but R-symmetry transformations can still act differently on the Weyl spinor modules. 
In matrix notation, invariance of the real superbracket requires

$$
\rho\left(R\left(\begin{array}{c}
\lambda_{+}^{j} \\
\lambda_{-}^{j}
\end{array}\right)\right)=R \rho\left(\begin{array}{c}
\lambda_{+}^{j} \\
\lambda_{-}^{j}
\end{array}\right) .
$$

Since the $\epsilon$-quaternionic structure flips chirality,

$$
R^{*}\left(\begin{array}{ll}
0 & L \\
L & 0
\end{array}\right)=\left(\begin{array}{ll}
0 & L \\
L & 0
\end{array}\right) R .
$$

Using that the complex R-symmetry group acts diagonally, we obtain

$$
\left(\begin{array}{cc}
0 & A^{*} L \\
B^{*} L & 0
\end{array}\right)=\left(\begin{array}{cc}
0 & L B \\
L A & 0
\end{array}\right),
$$

which leads to

$$
B=L^{-1} A^{*} L
$$

Thus, we see that

$$
R=\left(\begin{array}{cc}
A & 0 \\
0 & L^{-1} A^{*} L
\end{array}\right),
$$

where $A \in \mathrm{O}(K, \mathbb{C})$ or $\operatorname{Sp}(K, \mathbb{C})$.

This is a reducible representation of $\mathrm{O}(K, \mathbb{C})$ or $\operatorname{Sp}(K, \mathbb{C})$, given by the direct sum of the fundamental representation with a representation that is equivalent to the anti-fundamental representation (complex conjugate of the fundamental representation). Using table 2, it follows that the real R-symmetry group for orthogonal Weyl-incompatible signatures is, $\mathrm{O}(K, \mathbb{C}) \subset \mathrm{O}(K, \mathbb{C}) \times \mathrm{O}(K, \mathrm{C})$ in dimensions $D=2,10, \ldots$ (where both Majorana bilinear forms are super-admissible) and $\operatorname{Sp}(K, \mathbb{C}) \subset \operatorname{Sp}(K, \mathbb{C}) \times \operatorname{Sp}(K, \mathbb{C})$, in dimensions $D=6, \ldots$ (where both Majorana bilinear forms are anti-super-admissible). Note that due to the Weyl-incompatible reality condition we are restricted to the case $K_{+}=K_{-}=K$, and that the complexifications of $\mathrm{O}(K, \mathrm{C})$ and $\operatorname{Sp}(K, \mathbb{C})$, considered as a real Lie groups, are $\mathrm{O}(K, \mathbb{C}) \times \mathrm{O}(K, \mathbb{C})$ and $\operatorname{Sp}(K, \mathbb{C}) \times \operatorname{Sp}(K, \mathbb{C})$, respectively

\subsubsection{Isotropic Weyl-compatible signature}

Previously we found that for isotropic signatures R-symmetry transformations take the form

$$
R=\left(\begin{array}{cc}
A & 0 \\
0 & M^{-1}\left(A^{T}\right)^{-1} M
\end{array}\right)
$$

To simplify the following calculations we pass to the Lie algebra by setting $A=e^{a}$ and $R=e^{r}$, so that (6.12) takes the form

$$
r=\left(\begin{array}{cc}
a & 0 \\
0 & -M^{-1} a^{T} M
\end{array}\right) .
$$


In isotropic signatures, the two Majorana bilinear forms have opposite superadmissibility so that we need to consider both $M=\delta$ and $M=J$. We remark that we will see below that the R-symmetry group only depends on whether the $\epsilon$-quaternionic structure on $\mathbb{C}^{K}$ is a real or quaternionic structure, but is insensitive otherwise to the choice of $M$ and $L$.

We now specialise to Weyl-compatible signatures, where the reality condition preserves chirality, so that $r$ must satisfy

$$
r^{*}\left(\begin{array}{cc}
L & 0 \\
0 & L
\end{array}\right)=\left(\begin{array}{ll}
L & 0 \\
0 & L
\end{array}\right) r
$$

For $r$ given in (6.13) this implies

$$
a=L^{-1} a^{*} L, \quad M^{-1} a^{T} M=L^{-1}\left(M^{-1} a^{T} M\right)^{*} L .
$$

Rearranging the final equation in (6.15) we get

$$
a=\left(M L M^{-1}\right)^{-1} a^{*}\left(M L M^{-1}\right) .
$$

For $M=\delta$ we obviously have $M L M^{-1}=L$, and the same is true for $M=J$ for all possible choices Id, $\tilde{I}_{2 r, 2 s}, J$ for $L$. Therefore the only condition is

$$
a=L^{-1} a^{*} L
$$

In Weyl-compatible signatures the possible choices for $L$ are further restricted by the fact that both $\epsilon$-quaternionic structures on $\mathbb{S}$ have the same type.

Real structures on $\mathbb{S}$. We first consider the case where $\mathbb{S}$ carries two real structures. Then for $M=\delta$ we can have $L=\mathrm{Id}, I_{p, q}$ and for $M=J$ we can have $L=\mathrm{Id}, \tilde{I}_{2 r, 2 s}$.

For $L=\operatorname{Id}$ equation (6.17) directly tells us that $a \in \mathfrak{g l}(K, \mathbb{R})$, and we will now show that we obtain the same Lie algebra in all other cases. For $L=I_{p, q}$ we see that

$$
a=I_{p, q}^{-1} a^{*} I_{p, q} \Longrightarrow a=\left(\begin{array}{cc}
w & i x \\
i y & z
\end{array}\right),
$$

where $w$ is a $p \times p$ real matrix, $z$ is $q \times q, x$ is $p \times q$ and $y$ is $q \times p$. We see that $a$ is a $K \times K$ matrix depending on $K^{2}$ real numbers. Since the form of $a$ is preserved under matrix multiplication and hence under commutators, matrices of the form a define a $K^{2}$-dimensional real Lie subalgebra of $\mathfrak{g l}(K, \mathbb{C})$ which on dimensional grounds must be isomorphic to $\mathfrak{g l}(K, \mathbb{R})$. As already mentioned we will give explicit maps between different reality conditions later.

If $M=J$ we need to consider $L=\mathrm{Id}$ and $L=\tilde{I}_{2 r, 2 s}$. For $L=$ Id we see directly that $a \in \mathfrak{g l}(K, \mathbb{R})$. For $L=\tilde{I}_{2 r, 2 s}$ we find that $a$ must have the form

$$
a=\left(\begin{array}{cc}
W & X \\
Y & Z
\end{array}\right)
$$


where $W, X, Y, Z$ are $\frac{K}{2} \times \frac{K}{2}$ matrices that obey $V=I_{r, s} V^{*} I_{r, s}$ for $V=W, X, Y, Z$. Therefore all four blocks are of the form

$$
V=\left(\begin{array}{cc}
V_{1} & i V_{2} \\
i V_{3} & V_{4}
\end{array}\right)
$$

By the same reasoning as in the previous case, matrices of this structure form a real Lie subalgebra of $\mathfrak{g l}(K, \mathbb{C})$ which is isomorphic to $\mathfrak{g l}(K, \mathbb{R})$ for dimensional reasons. This means that $r \in \mathfrak{g l}(K, \mathbb{R})$ in isotropic Weyl-compatible signatures with real structures, regardless of the choice of $M$ and $L$. The R-symmetry group is $\operatorname{GL}(K, \mathbb{R})$.

Quaternionic structures on $\mathbb{S}$. In signatures where $\mathbb{S}$ has two quaternionic structures the only choice for $L$ is $L=J$. Matrices $a \in \mathfrak{g l}(K, \mathbb{C})$ which satisfy $(6.17)$ have the form

$$
a=\left(\begin{array}{cc}
x & y \\
-y^{*} & x^{*}
\end{array}\right), \quad x, y \in M_{\frac{K}{2}}(\mathbb{C}) .
$$

This defines the Lie subalgebra $\mathfrak{u}^{*}(K) \cong \mathfrak{g l}\left(\frac{K}{2}, \mathbb{H}\right)$ of $\mathfrak{g l}(K, \mathbb{C})$.

Upon exponentiation these matrices retain their form,

$$
A=e^{a}=\left(\begin{array}{cc}
X & Y \\
-Y^{*} & X^{*}
\end{array}\right), \quad X, Y \in \mathrm{GL}\left(\frac{K}{2}, \mathbb{C}\right) .
$$

The matrix $r$ is determined by $a$ and provides a reducible representation of $\mathfrak{u}^{*}(K)$. The R-symmetry group is $\mathrm{U}^{*}(K)$.

\subsubsection{Isotropic Weyl-incompatible signatures}

The last case are signatures where both the complex bilinear form and the reality condition pair opposite chiralities. Computations are conveniently carried out at the group level. To commute with the reality condition, $R$ must obey

$$
R^{*}\left(\begin{array}{ll}
0 & L \\
L & 0
\end{array}\right)=\left(\begin{array}{ll}
0 & L \\
L & 0
\end{array}\right) R
$$

Substituting in the form (6.12) that $\mathrm{R}$ takes in isotropic dimensions, this becomes

$$
\left(\begin{array}{cc}
0 & A^{*} L \\
M^{-1}\left(A^{\dagger}\right)^{-1} M L
\end{array}\right)=\left(\begin{array}{cc}
0 & L M^{-1}\left(A^{T}\right)^{-1} M \\
L A & 0
\end{array}\right) .
$$

This is two copies of the equation

$$
A^{\dagger}(M L) A=(M L)
$$

which defines the pseudo-unitary group $\mathrm{U}(p, q)$ where $(p, q)$ is the signature of the matrix $M L$. For some choices of $M$ and $L$ their product $M L$ will not be diagonal, so that matrices of the form $A$ provide a non-standard matrix realization of this group. Let us consider all possible combinations in turn. For $M=\delta$ the signature depends entirely on $L$ and is $(K, 0)$ for $L=\mathrm{Id},(p, q)$ for $L=I_{p, q}$ and $(k, k)$ for $L=J$ (where $K=2 k$ ). If either $M=J$ or 


\begin{tabular}{|c|c|c|c|}
\hline & $M$ & $L$ & $G_{R}$ \\
\hline \multirow{6}{*}{ WC } & $\delta$ & $\delta$ & $\mathrm{GL}(K, \mathbb{R})$ \\
\cline { 2 - 4 } & $\delta$ & $I_{p, q}$ & $\mathrm{GL}(K, \mathbb{R})$ \\
\cline { 2 - 4 } & $\delta$ & $J$ & $\mathrm{U}^{*}(K)$ \\
\cline { 2 - 4 } & $J$ & $\delta$ & $\mathrm{GL}(K, \mathbb{R})$ \\
\cline { 2 - 4 } & $J$ & $\tilde{I}_{2 r, 2 s}$ & $\mathrm{GL}(K, \mathbb{R})$ \\
\cline { 2 - 4 } & $J$ & $J$ & $\mathrm{U}^{*}(K)$ \\
\hline
\end{tabular}

\begin{tabular}{|c|c|c|c|}
\hline & $M$ & $L$ & $G_{R}$ \\
\hline \multirow{6}{*}{$\mathrm{WI}$} & $\delta$ & $\delta$ & $\mathrm{U}(K)$ \\
\cline { 2 - 4 } & $\delta$ & $I_{p, q}$ & $\mathrm{U}(p, q)$ \\
\cline { 2 - 4 } & $\delta$ & $J$ & $\mathrm{U}(k, k)$ \\
\cline { 2 - 4 } & $J$ & $\delta$ & $\mathrm{U}(k, k)$ \\
\cline { 2 - 4 } & $J$ & $\tilde{I}_{2 r, 2 s}$ & $\mathrm{U}(k, k)$ \\
\hline & $J$ & $J$ & $\mathrm{U}(K)$ \\
\hline
\end{tabular}

Table 6. In isotropic dimensions the R-symmetry groups are real forms of $\mathrm{GL}(K, \mathbb{C})$. Depending on whether the signature is Weyl-compatible (WC) or Weyl incompatible (WI), this table lists the R-symmetry groups for all possible combinations of a bilinear form $M$ with a reality condition $L$. Note that $K=2 k=2 r+2 s=p+q$.

$L=J$ (but not both), the matrix $M L$ is antisymmetric and therefore has purely imaginary eigenvalues. It can be diagonalized by a unitary transformation, and after pulling out a factor $i$ it defines a Hermitian form. The signature of this Hermitian form is $(k, k)$ for $L=\mathrm{Id}$ and $L=\tilde{I}_{2 r, 2 s}$, and $(K, 0)$ when $L=J$. In the last two cases we have made a choice of overall sign, which is conventional since $\mathrm{U}(p, q) \cong \mathrm{U}(q, p)$. Table 6 lists the R-symmetry groups for isotropic signatures.

\subsection{Real supersymmetry algebras and their R-symmetry groups}

In even dimensions R-symmetry groups vary much more than in odd dimensions, since we have to distinguish between orthogonal and isotropic dimensions, and between Weylcompatible and Weyl-incompatible signatures. In this section we provide tables for easy access, which expose patterns and provide information that one needs in applications. The first set of tables collects the supersymmetry algebras with a minimal number of supercharges. Recall that our convention is that $\mathcal{N}$, and $\mathcal{N}_{ \pm}$count supersymmetries in multiples of Majorana and Majorana-Weyl spinors, irrespective of whether such spinors, or a supersymmetry algebra based on such spinors, exist in the signature under consideration. In the next section we will show that the supersymmetry algebras constructed by our method are classified, up to the relative sign between $\alpha_{+}$and $\alpha_{-}$for orthogonal Weyl-compatible algebras, by their R-symmetry group. Therefore our tables provide a classification of supersymmetry algebras and not only of R-symmetry groups.

\subsection{1 $(1,0)$ or $(0,1)$ algebras}

In table 7 we list those signatures in even dimensions where the minimal superalgebras have $d_{\mathbb{S}} / 2$ real supercharges, where $d_{\mathbb{S}}$ is the complex dimension of the complex spinor module $\mathbb{S}$. In this case the supercharges form a single Majorana-Weyl spinor. This is only possible in orthogonal Weyl-compatible signatures, which can be read off from table 2, which in addition must admit Majorana-Weyl spinors, that is $t-s$ must be 0 modulo 8 , or, equivalently $\left(\epsilon_{+}, \epsilon_{-}\right)=(1,1)$ in table 1 . We denote these algebras as $\left(\mathcal{N}_{+}, \mathcal{N}_{-}\right)=(1,0),(0,1)$. The real 


\begin{tabular}{|c|c|c|c|c|c|c|c|}
\hline$D$ & $(0, D)$ & $(1, D-1)$ & $(2, D-2)$ & $(3, D-3)$ & $(4, D-4)$ & $(5, D-5)$ & $(6, D-6)$ \\
\hline 2 & - & $\mathbb{Z}_{2}$ & - & & & & \\
\hline 4 & - & - & - & - & - & & \\
\hline 6 & - & - & - & - & - & - & - \\
\hline 8 & - & - & - & - & - & - & - \\
\hline 10 & - & $\mathbb{Z}_{2}$ & - & - & - & $\mathbb{Z}_{2}$ & - \\
\hline 12 & - & - & - & - & - & - & - \\
\hline
\end{tabular}

Table 7. In this table we list chiral $\left(\mathcal{N}_{+}, \mathcal{N}_{-}\right)=(1,0),(0,1)$ supersymmetry algebras, where the supercharges form a single Majorana-Weyl spinor $\left(d_{\mathbb{S}} / 2\right.$ real supercharges, where $\left.d_{\mathbb{S}}=\operatorname{dim}_{\mathbb{C}} \mathbb{S}\right)$. Where such algebras exist, they are represented by their discrete R-symmetry group $O(1) \cong \mathbb{Z}_{2}$. A dash means no such algebra can be defined.

R-symmetry group is the discrete group $O(1)=\mathbb{Z}_{2}$, the same as for algebras based on a single Majorana spinor in odd dimensions.

In 10 dimensions the only signatures that allow a supersymmetry algebra with a single Majorana-Weyl supercharge are $(1,9)$ and $(5,5)$ (as well as $(9,1)$ ). In Lorentz signature these algebras are realized by type-I and heterotic string theories and the corresponding supergravity theories (which are usually denoted $\mathcal{N}=1$ ). Note that in many signatures which admit Majorana-Weyl spinors, no algebra based on a single Majorana-Weyl spinor exists. In particular, there are no $(1,0)$ and $(0,1)$ algebras in isotropic dimensions $D=4,8,12, \ldots$ Moreover, even in orthogonal dimensions, signatures where Majorana-Weyl spinors exist, such as $(t, s)=(3,3)$, may not admit $(1,0)$ and $(0,1)$ algebras, because none of the two Majorana bilinear forms are super-admissible. In this case the bilinear form on $\mathbb{C}^{K_{ \pm}}$is antisymmetric, which forces $K_{ \pm}$to be even and prevents one to define supersymmetry algebras based on a single irreducible spinor module. The corresponding orthogonal dimensions are $D=6,14, \ldots$, with complex R-symmetry groups $\operatorname{Sp}\left(K_{+}, \mathbb{C}\right) \times \operatorname{Sp}\left(K_{-}, \mathbb{C}\right)$ in table 2 .

\subsection{2 $(0,2),(1,1),(2,0)$ and $\mathcal{N}=1$ algebras}

We now turn to even-dimensional signatures which admit supersymmetry algebras with $d_{\mathbb{S}}$ real supercharges, which form a Majorana spinor or a Weyl spinor. The possible Rsymmetry groups are listed in table 8 . Chiral $(2,0)$ or $(1,1)$ supersymmetry algebras only exist in orthogonal dimensions $D=2,6,10, \ldots$, while supersymmetry algebras in isotropic dimension $D=4,8,12, \ldots$ are necessarily non-chiral and thus denoted $\mathcal{N}=1$.

In orthogonal dimensions, the Majorana bilinear forms are either both super-admissible $(D=2,10, \ldots)$ or both are anti-super-admissible $(D=6, \ldots)$. In Weyl-compatible signatures the $\epsilon$-quaternionic structures $J_{ \pm}^{(\epsilon)}$ on $\mathbb{S}_{ \pm}$are either both real or both quaternionic. For orthogonal Weyl-compatible signatures with a super-admissible Majorana bilinear forms and $J_{ \pm}^{(\epsilon)}$ both giving real structures we can define a $(1,1)$ superalgebra with R-symmetry $\mathbb{Z}_{2} \times \mathbb{Z}_{2}$ or $(2,0)$ superalgebras with R-symmetry group $\mathrm{O}(1,1)$ or $\mathrm{O}(2)$. If, however, we have super-admissible Majorana bilinear forms, but $J_{ \pm}^{(\epsilon)}$ are quaternionic structures on 


\begin{tabular}{|c|c|c|c|c|c|c|c|}
\hline$D$ & $(0, D)$ & $(1, D-1)$ & $(2, D-2)$ & $(3, D-3)$ & $(4, D-4)$ & $(5, D-5)$ & $(6, D-6)$ \\
\hline 2 & $\mathbb{Z}_{2}$ & $\mathrm{O}(1,1), \mathrm{O}(2), \mathbb{Z}_{2} \times \mathbb{Z}_{2}$ & $\mathbb{Z}_{2}$ & & & & \\
\hline 4 & - & $\mathrm{U}(1)$ & $\mathrm{SO}(1,1)$ & $\mathrm{U}(1)$ & - & & \\
\hline 6 & - & $\mathrm{SU}(2)$ & - & $\mathrm{SU}(1,1)$ & - & $\mathrm{SU}(2)$ & - \\
\hline 8 & $\mathrm{SO}(1,1)$ & $\mathrm{U}(1)$ & - & $\mathrm{U}(1)$ & $\mathrm{SO}(1,1)$ & $\mathrm{U}(1)$ & - \\
\hline 10 & $\mathbb{Z}_{2}$ & $\mathrm{O}(1,1), \mathrm{O}(2), \mathbb{Z}_{2} \times \mathbb{Z}_{2}$ & $\mathbb{Z}_{2}$ & $\mathrm{SO}(2)$ & $\mathbb{Z}_{2}$ & $\mathrm{O}(1,1), \mathrm{O}(2), \mathbb{Z}_{2} \times \mathbb{Z}_{2}$ & $\mathbb{Z}_{2}$ \\
\hline 12 & - & $\mathrm{U}(1)$ & $\mathrm{SO}(1,1)$ & $\mathrm{U}(1)$ & - & $\mathrm{U}(1)$ & $\mathrm{SO}(1,1)$ \\
\hline
\end{tabular}

Table 8. R-symmetry groups for $\mathcal{N}=1$ and $\left(\mathcal{N}_{+}, \mathcal{N}_{-}\right)=(2,0),(1,1),(0,1)$ supersymmetry algebras with $d_{\mathbb{S}}$ real supercharges in even dimensions. A dash indicates signatures where no such supersymmetry algebra exists.

$\mathbb{S}_{ \pm}$, then we can only define a $(2,0)$ superalgebra with $\mathrm{SO}(2)$ R-symmetry. ${ }^{24}$ When the Majorana bilinear forms are anti-super-admissible we can only define a $(2,0)$ algebra, with R-symmetry group given by $\mathrm{SU}(2)$ if $J^{(\epsilon)_{ \pm}}$are quaternionic structures or $\mathrm{SU}(1,1)$ if they are real structures.

Orthogonal Weyl-incompatible signatures can only have a $(1,1)$ superalgebra which therefore needs a super-admissible Majorana bilinear form. The result is a $\mathbb{Z}_{2}$ R-symmetry group because the reality condition links the R-symmetry transformations on the two Weyl spinor modules.

Consider finally the non-chiral $\mathcal{N}=1$ algebras in isotropic dimensions. Here the R-symmetry group is $\mathrm{SO}(1,1)$ in isotropic Weyl-compatible signatures, and $\mathrm{U}(1)$ in isotropic Weyl-incompatible signatures.

Let us consider a few examples for illustration. In signature $(1,9)$ the supersymmetry algebra with $\mathbb{Z}_{2} \times \mathbb{Z}_{2}$ R-symmetry is that of Type IIA or IIA* theories. These have the same R-symmetry group but opposite relative signs $\alpha_{+}= \pm \alpha_{-}$, as is further discussed in section 8.1. The supersymmetry algebra with $\mathrm{O}(2)$ R-symmetry is realized in type-IIB supergravity and string theory, and that with $\mathrm{O}(1,1)$ R-symmetry in type-IIB*.

In signature $(1,5)$, which is orthogonal and Weyl compatible, the entry $\mathrm{SU}(2)$ represents the minimal supersymmetry algebra, which is a chiral $(2,0)$ algebra based on a single Weyl spinor, which for this signature is the same as a real semi-spinor $\left(\mathbb{S}_{ \pm} \cong S_{ \pm}, S_{+} \nsucceq S_{-}\right)$. This algebra is usually denoted $(1,0)$ in the literature, but with our conventions the notation $(1,0)$ is reserved for algebras based on Majorana-Weyl spinors, which do not exist in this signature. In four dimensions we see the standard minimal supersymmetry algebra in Lorentz signature $(1,3)$ or $(3,1)$ with R-symmetry group $\mathrm{U}(1)$. The minimal supersymmetry algebra in neutral signature $(2,2)$ has R-symmetry group $\mathrm{SO}(1,1)$. All these algebras are based on Majorana spinors. Note that while Majorana-Weyl spinors exist in signature $(2,2)$, there is no $\left(\mathcal{N}_{+}, \mathcal{N}_{-}\right)=(1,0)$ supersymmetry algebra, since four is an isotropic dimension and the restriction of the $\mathcal{N}=1$ superbracket to Majorana-Weyl spinors is degenerate, as already observed in [17].

\footnotetext{
${ }^{24}$ The quaternionic structure fixes the orientiation of the auxiliary space, which reduces the R-symmetry group from $\mathrm{O}(2)$ to $\mathrm{SO}(2)$.
} 


\begin{tabular}{|c|c|c|c|c|c|c|c|}
\hline$D$ & $(0, D)$ & $(1, D-1)$ & $(2, D-2)$ & $(3, D-3)$ & $(4, D-4)$ & $(5, D-5)$ & $(6, D-6)$ \\
\hline 2 & $\mathrm{O}(\mathcal{N}, \mathbb{C})$ & $\mathrm{O}\left(p_{+}, q_{+}\right) \times \mathrm{O}\left(p_{-}, q_{-}\right)$ & $\mathrm{O}(\mathcal{N}, \mathbb{C})$ & & & & \\
\hline 4 & $\mathrm{U}^{*}(\mathcal{N})$ & $\mathrm{U}(p, q)$ & $\mathrm{GL}(\mathcal{N} \mathbb{R})$ & $\mathrm{U}(p, q)$ & $\mathrm{U}^{*}(\mathcal{N})$ & & \\
\hline 6 & $\mathrm{Sp}(\mathcal{N}, \mathbb{C})$ & $\mathrm{USp}\left(\mathcal{N}_{+}\right) \times \mathrm{USp}\left(\mathcal{N}_{-}\right)$ & $\mathrm{Sp}(\mathcal{N}, \mathbb{C})$ & $\mathrm{X}$ & $\mathrm{Sp}(\mathcal{N}, \mathbb{C})$ & $\left.\mathrm{USp}\left(\mathcal{N}_{+}\right) \times \mathrm{USp}(\mathcal{N})_{-}\right)$ & $\mathrm{Sp}(\mathcal{N}, \mathbb{C})$ \\
\hline 8 & $\mathrm{GL}(\mathcal{N}, \mathbb{R})$ & $\mathrm{U}(p, q)$ & $\mathrm{U}^{*}(\mathcal{N})$ & $\mathrm{U}(p, q)$ & $\mathrm{GL}(\mathcal{N}, \mathbb{R})$ & $\mathrm{U}(p, q)$ & $\mathrm{U}^{*}(\mathcal{N})$ \\
\hline 10 & $\mathrm{O}(\mathcal{N}, \mathbb{C})$ & $\mathrm{O}\left(p_{+}, q_{+}\right) \times \mathrm{O}\left(p_{-}, q_{-}\right)$ & $\mathrm{O}(\mathcal{N}, \mathbb{C})$ & $\mathrm{SO}^{*}\left(\mathcal{N}_{+}\right) \times \mathrm{SO}^{*}\left(\mathcal{N}_{-}\right)$ & $\mathrm{O}(\mathcal{N}, \mathbb{C})$ & $\mathrm{O}\left(p_{+}, q_{+}\right) \times \mathrm{O}\left(p_{-}, q-\right)$ & $\mathrm{O}(\mathcal{N}, \mathbb{C})$ \\
\hline 12 & $\mathrm{U}^{*}(\mathcal{N})$ & $\mathrm{U}(p, q)$ & $\mathrm{GL}(\mathcal{N}, \mathbb{R})$ & $\mathrm{U}(p, q)$ & $\mathrm{U}^{*}(\mathcal{N})$ & $\mathrm{U}(p, q)$ & $\mathrm{GL}(\mathcal{N}, \mathbb{R})$ \\
\hline
\end{tabular}

Table 9. R-symmetry groups possible in even dimension with any signature. Note that $p_{+}+q_{+}=\mathcal{N}_{+}$, $p_{-}+q_{-}=\mathcal{N}_{-}$and $\mathcal{N}_{+}+\mathcal{N}_{-}=2 \mathcal{N} . \quad X=\left(\operatorname{Sp}\left(\mathcal{N}_{+}, \mathbb{R}\right)\right.$ or $\left.\operatorname{USp}\left(2 r_{+}, 2 s_{+}\right)\right) \times\left(\operatorname{Sp}\left(\mathcal{N}_{-}, \mathbb{R}\right)\right.$ or $\left.\mathrm{USp}\left(2 r_{-}, 2 s_{-}\right)\right)$.

Any signatures without an entry in this table have a minimal superalgebra with $2 d_{\mathbb{S}}$ real supercharges, that is the supercharges form a Dirac spinor. With our convention these are $(2,2)$ superalgebras in orthogonal dimensions, and $\mathcal{N}=2$ algebras in isotropic dimensions. For example, in spacetime signature $(t, s)=(0,4)$ the minimal supersymmetry algebra is the $\mathcal{N}=2$ algebra.

\subsubsection{General $\left(\mathcal{N}_{+}, \mathcal{N}_{-}\right)$}

We conclude this section by providing further tables for reference. Table 9 lists the Rsymmetry groups for general extended supersymmetry algebras in even dimensions and arbitrary signature. This is followed by a master table, table 10, which combines our results on R-symmetry groups in even and odd dimensions.

\section{Isomorphisms and classification}

So far we have shown that we can construct a real supersymmetry algebra given the following data: a complex bilinear form $C \otimes M$ on the complex extended spinor module $\mathfrak{g}_{1}^{\mathbb{C}}=\mathbb{S} \otimes \mathbb{C}^{K}$, a real structure $\rho$ on this space, which is determined by an $\epsilon$-quaternionic structure $B$ on the complex spinor module $\mathbb{S}$, and a map $L$ on the auxiliary space $\mathbb{C}^{K}$, which determines a real form of the complex R-symmetry group. In orthgonal Weyl-compatible signatures we can choose these data independently for the chiral sectors. While algebras with distinct R-symmetry groups cannot be isomorphic, there is, to our knowledge, no rigorous statement asserting the converse, that is, that supersymmetry algebras are classified by their R-symmetry groups. Still, by inspection of our classification tables, we observe that different sets of data $(C, M, B, L)$ on $\mathfrak{g}_{1}^{\mathbb{C}}$ often lead to the same R-symmetry group, and previous experience tells one that in such cases one can often construct isomorphisms between the corresponding supersymmetry algebras. See in particular [17] on which we will now elaborate to show that, with one qualification applying to the orthogonal Weylcompatible case, the supersymmetry algebras which we have constructed in this paper are classified by their R-symmetry groups. We proceed by discussing each of the five cases in turn, and investigate which isomorphisms need to exist in order to relate all supersymmetry algebras with the same R-symmetry group to each other. Details of these isomorphisms are given in appendix E. 


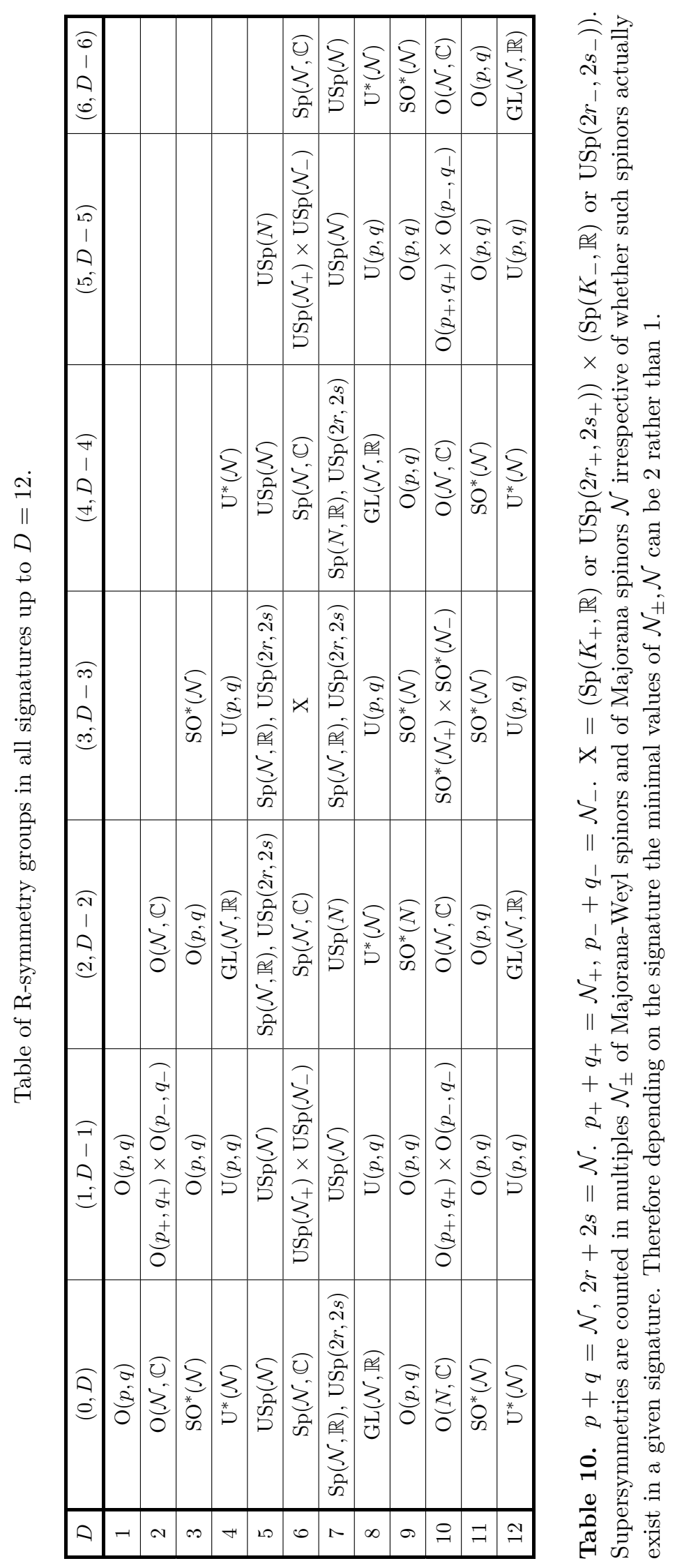




\subsection{Odd dimensions}

In odd dimensions there is only one inequivalent choice for $C$, which fixes $M$. Thus complex supersymmetry algebras with a given odd part $\mathfrak{g}_{1}^{\mathbb{C}}$ are unique, and in particular classified by their R-symmetry group. For real supersymmetry algebras we have to pick a reality condition, which is defined by a choice of $L$ which is compatible with our choice of $B$. Since $B$ is unique, real supersymmetry algebras are classified by the choice of $L$, that is by their R-symmetry group.

\subsection{Orthogonal, Weyl-compatible signatures}

In orthogonal dimensions, $C_{ \pm}$are either both super-admissible or both are not, which means that $M$ is fixed by the dimension. It remains to be seen whether the choice of $C_{ \pm}$has an effect. As far as complex supersymmetry algebras are concerned, showing that $C_{ \pm}$lead to isomorphic supersymmetry algebras implies that these are classified by their R-symmetry group. For real supersymmetry algebras we have to choose a $B$-matrix. In Weyl-compatible signatures $B_{ \pm}$are either both real structures or both quaternionic structures, and therefore they combine with the same set of $L$ 's to define a real structure. To show that real supersymmetry algebras are classified by the choice of $L$, we need to show that we can replace $B_{ \pm}$by $B_{\mp}$ in the reality condition without changing the complex bilinear form. We also need to show that we can replace $C_{ \pm}$by $C_{\mp}$ without changing the reality condition.

To be precise, an additional complication arises because there are two independent phase factors $\alpha_{ \pm}$in the reality conditions for the two chiral sectors. As we discussed before, imposing reality of the superbracket fixes these factors to be either \pm 1 or $\pm i$. While the overall sign of the pair $\alpha_{ \pm}$corresponds to an isomorphism of supersymmetry algebras, the relative sign distinguishes non-isomorphic supersymmetry algebras, in particular the IIA and IIA* algebras in ten dimensions, compare section 8.1. This is a particular feature of the orthogonal Weyl-compatible case, where both the superbracket and the reality condition are compatible with chirality. Therefore the best statement we can aim for is that real supersymmetry algebras are classified by their R-symmetry group together with a choice of the relative sign between $\alpha_{+}$and $\alpha_{-}$.

As shown in appendix E the R-transformation (E.1) maps the orthogonal bilinear forms $C_{ \pm} \otimes M$ to one another, while preserving the reality condition up to an irrelevant overall phase factor. Moreover, (A.25) implies that in orthogonal dimensions $B_{ \pm} \gamma_{*}=\mp i B_{\mp}$ and therefore the reality condition $\left(\lambda_{ \pm}^{i}\right)^{*}=\alpha_{ \pm} B_{-} \lambda_{ \pm}^{j} L_{j i}$ is equivalent to the reality condition $\left(\lambda_{ \pm}^{i}\right)^{*}= \pm i \alpha_{ \pm} B_{+} \lambda_{ \pm}^{j} L_{j i}$. This shows that the choice of $B_{ \pm}$only matters to the extent that it is correlated with the relative sign between $\alpha_{+}$and $\alpha_{-}$. In other words the real supersymmetry algebra only depends on the choice of $L$, that is on its real R-symmetry group, and on the relative sign between $\alpha_{+}$and $\alpha_{-}$. For complex supersymmetry algebras the choice of reality conditions and thus of relative signs is irrelevant, and we note as a corrolary that complex supersymmetry algebras in orthogonal dimensions are classified by their R-symmetry groups. 


\subsection{Orthogonal, Weyl-incompatible signatures}

Here we again have two options $C_{ \pm}$for $C$, while $M$ is fixed by dimension. Concerning reality conditions, one of $B_{ \pm}$defines a real, the other a quaternionic structure and therefore the choice of $B$ is determined by $L$ being a real or quaternionic structure. We have shown in section 6.1.3 that the R-symmetry group is $\mathrm{O}(K, \mathbb{C})$ if $C_{ \pm}$are super-admissible, and $M$ is symmetric, while it is $\operatorname{Sp}(K, \mathbb{C})$ if $C_{ \pm}$are not super-admissible and $M$ is antisymmetric. Since the R-symmetry group is determined by the dimension, we need to show that all possible choices of $C, L$ are equivalent. In appendix $\mathrm{E}$ we show that the R-transformation (E.1) exchanges the orthogonal bilinear forms, while preserving Weyl-incompatible reality conditions. Moreover, we show in appendix E that we can use the transformation $S_{L^{-1}}$ defined in (E.11) to map any reality condition to the one with $L=\mathrm{Id}$. For a given $M$, the canonical choices for $L$ were specified in section 4.2. For these $L$-matrices, the bilinear form is invariant under $S_{L^{-1}}$, or, in the case $M=J, L=I_{1,1}$ anti-invariant, which can be compensated by an overall phase in the reality condition.

\subsection{Isotropic, Weyl-compatible signatures}

In isotropic dimensions $C_{ \pm}$have opposite super-admissibility properties. The choice of $C$ determines the choice of $M$, which for complex superalgebras implies that they are classified by their R-symmetry group. If the signature is Weyl-compatible, then $B_{ \pm}$either both define a real or both define a quaternionic structure, and are thus compatible with the same set of $L$ 's. According to our classification, there are two possible R-symmetry groups: if $L$ (and thus $B_{ \pm}$) defines a real structure, the R-symmetry group is $\operatorname{GL}(K, \mathbb{R})$, if $L$ defines a quaternionic structure, then the R-symmetry group is $\mathrm{U}^{*}(K)$. Thus the $\mathrm{R}$-symmetry group is determined by the signature. According to table 6 there are four combinations of $(M, L)$ with R-symmetry group $\mathrm{GL}(K, \mathbb{R})$ and two combinations of $(M, L)$ with R-symmetry group $\mathrm{U}^{*}(K)$. To show that real supersymmetry algebras are classified by their R-symmetry groups the following statements are sufficient:

1. For $L=\delta$ and $L=J$ the bilinear forms $C \otimes \delta$ and $C^{\prime} \otimes J$, define isomorphic supersymmetry algebras, where $C$ denotes the super-admissible $C$-matrix and $C^{\prime}$ the non-super admissible $C$-matrix. This can be done using the transformation $S_{J}$ defined in appendix E.2, which exchanges the bilinear forms while preserving $L=\delta$ and $L=J$.

2. For $C \otimes \delta$, the reality conditions with $L=\delta$ and $L=I_{p, q}$ define isomorphic supersymmetry algebras, while for $C^{\prime} \otimes J$ the reality conditions with $L=\delta$ and $L=\tilde{I}_{2 r, 2 s}$ define isomorphic supersymmetry algebras. This can be done using the transformation $T$, defined in appendix E.3 which maps the $L$ 's as required, while preserving the corresponding bilinear forms.

3. The $R$-transformation, defined in appendix E.1 exchanges $B_{ \pm}$without changing the bilinear form. Therefore it does not matter which $B$ we use in the reality condition. 


\subsection{Isotropic, Weyl-incompatible signatures}

In this case $C_{ \pm}$have opposite super-admissibility and $B_{ \pm}$have opposite $\epsilon$-type. Therefore $(C, B)$ are determined by $(M, L)$. By inspection of table 6 there are seven distinct combinations of $(M, L)$ which correspond to only three distinct types of R-symmetry groups, $\mathrm{U}(K)$, $\mathrm{U}(p, q)$, with $p \neq q$, and $\mathrm{U}(k, k)$. To show that real supersymmetry algebras are classified by their R-symmetry groups, we need to show that

1. R-symmetry group $\mathrm{U}(K)$ : real supersymmetry algebras defined using $(M, L)=$ $(\delta, \delta),(J, J)$ are isomorphic.

2. R-symmetry group $\mathrm{U}(k, k)$ : real supersymmetry algebas defined using $(M, L)=$ $(\delta, J),\left(\delta, I_{k, k}\right),(J, \delta),\left(J, \tilde{I}_{2 r, 2 s}\right)$ are isomorphic.

These statements can be proved using the transformations $S_{J}, F, G$ as indicated in the following diagram:

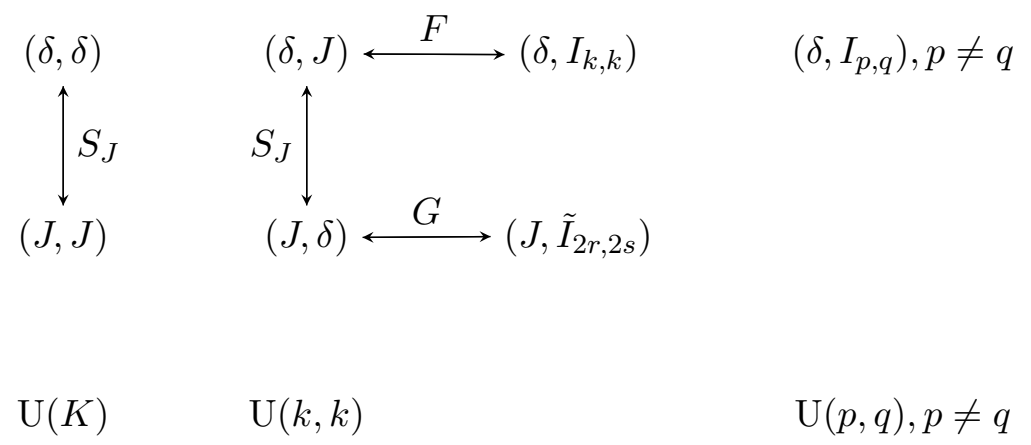

Details are given in appendix E.4.

\subsection{Remarks on the general classification problem}

In this paper we have constructed Poincaré Lie superalgebras with odd part $\mathfrak{g}_{1}$ by specifying a discrete set $(C, M, B, L)$ of data, which is subject to certain consistency conditions. Within this construction we have shown that the resulting supersymmetry algebras are classified by their R-symmetry groups, together with a choice of the relative sign between $\alpha_{+}$and $\alpha_{-}$for orthogonal Weyl-compatible algebras. However, this does not necessarily provide a classification of $\mathcal{N}$-extended Poincaré Lie superalgebras in arbitrary signature up to isomorphism. The general classification problem can be stated as follows. Firstly, for a given $\mathfrak{g}_{1}$ one needs to find the space of all superbrackets, that is of symmetric, vector-valued $\operatorname{Spin}(t, s)$-equivariant bilinear forms. This problem was solved in [22], where explicit bases in terms of super-admissible bilinear forms have been constructed. Secondly, one needs a criterion which allows one to decide when two symmetric, vector-valued $\operatorname{Spin}(t, s)$-equivariant bilinear forms define isomorphic Poincaré Lie superalgebras. This question is answered by Theorem 1 of [17], which specifies necessary and sufficient conditions for two Poincaré Lie superalgebras to be isomorphic. Up to checking certain discrete transformations, the classification up to isomorphism amounts to identifying the orbits of the Schur group on the 
space of symmetric vector-valued $\operatorname{Spin}(t, s)$-equivariant bilinear forms. The third step is the classification of Schur group orbits for the spaces of superbrackets known from [22] (together with checking for additional identifications by elements of $\operatorname{Pin}(t, s) \backslash \operatorname{Spin}(t, s)$, see [17] for details). Due to the mod 8 periodicity of the table of Clifford algebras, which imprints itself on the data defining supersymmetry algebras, this is a finite problem. The classification was carried out for algebras based on the complex spinor module $\mathbb{S}(\mathcal{N}=2)$ in five and four space-time dimensions in $[16,17]$. In this case the algebras in a given signature are indeed classified by their R-symmetry groups, which is encouraging, and this may well be true in general. However, there are potential subtleties which make the full classification problem somewhat involved. Non-degenerate superbrackets correspond to open orbits of the Schur group on the space of superbrackets, and the R-symmetry group is the stabilizer group of the orbit. It may happen that two connected open orbits have the same stabilizer, in which case one needs to carefully check whether there exists a discrete transformation relating these two orbits which provides an isomorphism. Comparing to the work presented in the paper this relates to the question whether the discrete data we use samples all orbits of the Schur group. A priori, there could be open orbits with no representatives within our construction, though we have no evidence that this is the case.

\section{Applications to type-II string theories in $D=10,9,4,3$}

\section{1 $\mathcal{N}=2$ supersymmetry in signature $(1,9)$ and type-II string theories}

We start in signature $(1,9)$ which is orthogonal Weyl-compatible, with two super-admissible bilinear forms $C_{ \pm}$and two real structures $B_{ \pm}$on $\mathbb{S}$. This signature admits two $(1,1)$ algebras with R-symmetry $\mathbb{Z}_{2} \times \mathbb{Z}_{2}$, which are distinguished by the relative sign $\alpha_{-}= \pm \alpha_{+}$. We can choose either of the $C_{ \pm}$to define the Majorana bilinear form and, independently, either of the $B_{ \pm}$to define a reality condition, but all choices lead to equivalent real superbrackets. For definiteness we choose $C_{+}, B_{+}$in the following. The complex vector-valued bilinear form is

$$
\left(\Gamma^{\mu} \lambda\right)^{T} C_{+} \chi=\left(\Gamma^{\mu} \lambda_{+}\right)^{T} C_{+} \chi_{+}+\left(\Gamma^{\mu} \lambda_{-}\right)^{T} C_{+} \chi_{-},
$$

where $\lambda_{ \pm}, \chi_{ \pm} \in \mathbb{S}_{ \pm}$. Following the conventions of appendix $\mathrm{F}$ we denote the ten-dimensional Dirac matrices by $\Gamma^{\mu}$. To select the type-IIA or type-IIA* algebra, we impose the reality conditions

$$
\left(\lambda_{+}\right)^{*}=\alpha B_{+} \lambda_{+}, \quad\left(\lambda_{-}\right)^{*}= \pm \alpha B_{+} \lambda_{-} .
$$

The + sign is the standard Majorana condition, while the - sign corresponds to a twisted Majorana condition which selects the type-IIA* algebra. We remark that with our conventions the complex bilinear form is fixed, and type-IIA and type-IIA* are distinguished by their reality conditions. One could equivalently impose the same reality condition, but define the type-IIA* theory using a modified complex superbracket. This amounts to $\lambda_{-} \rightarrow i \lambda_{-}$, and we will come back to this option below. For reference, let us also write down how the vector-valued bilinear form (8.1) translates into anti-commutators of supercharges:

$$
\left\{Q_{+, \alpha}, Q_{+, \beta}\right\}=\left(\Gamma^{\mu} C_{+}\right)_{\alpha \beta} P_{\mu}, \quad\left\{Q_{-, \alpha^{\prime}}, Q_{-, \beta^{\prime}}\right\}=\left(\Gamma^{\mu} C_{+}\right)_{\alpha^{\prime} \beta^{\prime}} P_{\mu}
$$


where the spinor indices $\alpha, \beta, \ldots$ and $\alpha^{\prime}, \beta^{\prime}, \ldots$ refer to $\mathbb{S}_{ \pm}$. In the following we will prefer to work with vector-valued bilinear forms as this allows us to suppress spinor indices.

Signature $(1,9)$ also admits chiral $\mathcal{N}=2$ algebras, and for definiteness we take $\left(\mathcal{N}_{+}, \mathcal{N}_{-}\right)=(2,0)$. The complex superbracket takes the form

$$
\left(\Gamma^{\mu} \lambda_{+}^{i}\right)^{T} C_{+} \chi_{+}^{j} M_{j i}, \quad M_{i j}=\delta_{i j},
$$

and the reality conditions are

$$
\left(\lambda_{+}^{i}\right)^{*}=\alpha B_{+} \lambda_{+}^{j} L_{j i}, \quad L_{i j}=\left(\begin{array}{cc}
1 & 0 \\
0 & \pm 1
\end{array}\right) .
$$

Depending on the choice of the sign in $L_{i j}$, we obtain the type-IIB algebra with R-symmetry group $\mathrm{O}(2)$ or the type-IIB* algebra with R-symmetry group $\mathrm{O}(1,1)$.

As is well known, the type-IIA/IIB algebras are realized in the type-IIA/IIB string theories, whose massless sectors are described by type-IIA/IIB supergravity, and these two string theories are related by T-duality. Moreover, it was shown in [1] that timelike T-duality maps type-IIA/IIB string theory to type-IIB*/IIA* string theory. The corresponding typeII* supergravity theories differ from their type-II counter parts by a sign flip of the kinetic terms for all Ramond-Ramond fields, as well as by factors of $i$ in their fermionic terms.

Apart from showing immediately the (potential) existence of type-II* theories, our formalism also makes it straightforward to show how these algebras are related to one another by T-duality. Two superstring theories are related by T-duality if theory $A$ on the background $\mathbb{R}^{t^{\prime}, s^{\prime}} \times S_{R}^{1}$ is identical to theory $B$ on the background $\mathbb{R}^{t^{\prime}, s^{\prime}} \times S_{1 / R}^{1}$, where the radius $R$ of the circle is measured in string units, and $t^{\prime}+s^{\prime}=9$. To include timelike T-duality, we allow the circle to be timelike. The ten-dimensional theories $A, B$ which arise in the two decompactification limits $R \rightarrow \infty$ and $R^{\prime}=1 / R \rightarrow \infty$ are then also said to be T-dual to each other. Note that this does not imply that they are equivalent as theories on $\mathbb{R}^{t, s}, t+s=10$. In particular, type-IIA and type-IIB string theory are distinct as theories on $\mathbb{R}^{1,9}$, and in particular have supersymmetry algebras which are not isomorphic to each other. Thus at the level of supersymmetry algebras T-duality is a map between supersymmetry algebras, but in general is not an isomorphism. It relates ten-dimensional supersymmetry algebra which upon dimensional reduction 'contract' to the same nine-dimensional algebra, a fact that we will explore further in section 8.3.

In the following we use that in theories of closed strings T-duality can be viewed as a 'chiral reflection,' which only acts on the (say) the right-moving degrees of freedom. In particular, on a ten-dimensional spinor $\lambda$, T-duality acts by

$$
\mathcal{T}: \quad \lambda_{+} \rightarrow \tilde{\lambda}_{+}=\lambda_{+}, \quad \lambda_{-} \rightarrow \tilde{\lambda}_{-}=T \lambda_{-}, \quad T=\beta \Gamma_{*} \Gamma^{9 / 0}, \quad|\beta|=1,
$$

with $\Gamma^{9}$ for spacelike and $\Gamma^{0}$ for timelike T-duality. We have introduced an arbitrary phase $\beta$ which will be used later to interpolate between different conventions for supersymmetry algebras.

We start from the type-IIA/IIA* algebras which are based on a spinor $\lambda$ subject to the reality condition (8.2). We note that T-duality maps $\mathbb{S}_{+} \oplus \mathbb{S}_{-} \rightarrow \mathbb{S}_{+} \oplus \mathbb{S}_{+}$, and therefore 
the image of a non-chiral type-IIA/IIA* supersymmetry algebra will be a chiral $(2,0)$ supersymmetry algebra.

The matrix $T$ is unitary for both the spacelike and the timelike case. To verify that it acts as a reflection in the 9-direction and in the 0-direction, respectively, we compute

$$
T \Gamma^{\mu} T^{-1}=\left\{\begin{array}{r}
-\Gamma^{\mu}, \text { for } \mu=9 / 0, \\
\Gamma^{\mu}, \text { for } \mu \neq 9 / 0,
\end{array}\right\}=\tilde{\Gamma}^{\mu} .
$$

Since T-duality maps the matrices $\Gamma^{\mu}$ to a new set $\tilde{\Gamma}^{\mu}$, this gives rise to new matrices $A$ and $C:^{25}$

$$
A \rightarrow \tilde{A}=T^{\dagger,-1} A T^{-1}=T A T^{\dagger}, \quad C_{+} \rightarrow \tilde{C}_{+}=T^{T,-1} C_{+} T^{-1} .
$$

We compute: ${ }^{26}$

spacelike T-duality: $\quad \tilde{C}_{+}=\frac{1}{\beta^{2}} C_{+}, \quad \tilde{A}=A, \quad \tilde{B}_{+}=\left(\tilde{C}_{+} \tilde{A}^{-1}\right)^{T}=\frac{1}{\beta^{2}} B_{+}$,

timelike T-duality: $\quad \tilde{C}_{+}=-\frac{1}{\beta^{2}} C_{+}, \quad \tilde{A}=-A, \quad \tilde{B}_{+}=\left(\tilde{C}_{+} \tilde{A}^{-1}\right)^{T}=\frac{1}{\beta^{2}} B_{+}$.

Thus the difference between spacelike and timelike T-duality is a relative sign between the transformation behaviour of $C_{+}$and $B_{+}$, which induces a corresponding sign in the relation between the complex bilinear form and the reality condition.

To see this explicitly, we express $\lambda_{-}, \chi_{-}$in terms of the transformed spinors $\tilde{\lambda}_{-}, \tilde{\chi}_{-}$in the bilinear form

$$
\begin{aligned}
\left(\Gamma^{\mu} \lambda_{-}\right)^{T} C_{+} \chi_{-} & =\left(\Gamma^{\mu} T^{-1} \tilde{\lambda}_{-}\right)^{T} C_{+} T^{-1} \tilde{\chi}_{-}=\left(\tilde{\Gamma}^{\mu} \tilde{\lambda}_{-}\right)^{T} T^{-1, T} C_{+} T^{-1} \tilde{\chi}_{-} \\
& =\left(\tilde{\Gamma}^{\mu} \tilde{\lambda}_{-}\right)^{T} \tilde{C}_{+} \tilde{\chi}_{-}= \pm \frac{1}{\beta^{2}}\left(\tilde{\Gamma}^{\mu} \tilde{\lambda}_{-}\right)^{T} C_{+} \tilde{\chi}_{-}
\end{aligned}
$$

The upper sign refers to spacelike, the lower sign to timelike T-duality.

Since the transformed spinors both have positive chirality, we prefer to use the notation $\left(\lambda_{+}^{1}, \lambda_{+}^{2}\right)$ instead of $\left(\tilde{\lambda}_{+}, \tilde{\lambda}_{-}\right)$, and we also relabel $\tilde{\Gamma}^{\mu} \rightarrow \Gamma^{\mu}$, though this change needs to be taken into account in the reality condition satisfied by $\lambda_{+}^{2}$, see below. Combining both chiral sectors, the new complex vector-valued bilinear form is

$$
\left(\Gamma^{\mu} \lambda_{+}^{1}\right)^{T} C_{+} \chi_{+}^{1} \pm \frac{1}{\beta^{2}}\left(\Gamma^{\mu} \lambda_{+}^{2}\right)^{T} C_{+} \chi_{+}^{2}=\left(\Gamma^{\mu} \lambda_{+}^{i}\right)^{T} C_{+} \chi_{+}^{j} M_{j i}, \quad M_{i j}=\left(\begin{array}{cc}
1 & 0 \\
0 & \pm \frac{1}{\beta^{2}}
\end{array}\right),
$$

with $+/$ - for spacelike/timelike T-duality. The reality condition (8.2) is mapped to the new reality condition

$$
\left(\tilde{\lambda}_{+}^{i}\right)^{*}=\alpha_{(i)} \tilde{B}_{+} \tilde{\lambda}_{+}^{i}=\alpha B_{+} \tilde{\lambda}_{+}^{j} L_{j i}, \quad L_{i j}=\left(\begin{array}{cc}
1 & 0 \\
0 & \epsilon \frac{1}{\beta^{2}}
\end{array}\right),
$$

\footnotetext{
${ }^{25} A$ is the matrix defining the Dirac Hermitian sesquilinear form. In signature $(1, D-1)$ we take $A=\Gamma_{0}$.

${ }^{26}$ The sign in the relation for $C_{+}$depends on that we have chosen $C_{+}$rather than $C_{-}$, since we need to make use of the relation $\tau_{+}=-1$.
} 
where we used that $\tilde{B}_{+}=B_{+}$when acting on $\lambda_{+}^{1}=\tilde{\lambda}_{+}$, while $\tilde{B}_{+}=\frac{1}{\beta^{2}} B_{+}$when acting on $\lambda_{+}^{2}=\tilde{\lambda}_{-}$. To account for the phases in the original reality condition (8.2) we have set $\alpha_{(1)}=\alpha_{+}=\alpha, \alpha_{(2)}=\alpha_{-}=\epsilon \alpha$, with $\epsilon=1$ if we start with type-IIA and with $\epsilon=-1$ if we start with type-IIA*.

There are two natural choices for the phase $\beta$.

1. We can choose $\beta$ such that the complex bilinear form has the canonical form $M_{i j}=\delta_{i j}$, which is the convention used in this paper. This requires $\beta= \pm 1$ for spacelike and $\beta= \pm i$ for timelike T-duality. In this case the real supersymmetry algebras are distinguished by their reality conditions:

$$
\left(M_{i j}\right)=\left(\begin{array}{ll}
1 & 0 \\
0 & 1
\end{array}\right), \quad\left(L_{i j}\right)=\left(\begin{array}{cc}
1 & 0 \\
0 & \pm \epsilon
\end{array}\right) .
$$

This shows that the type-IIA algebra, $\epsilon=1$, is mapped by spacelike T-duality to the type-IIB algebra with R-symmetry group $\mathrm{O}(2)$, and by timelike T-duality to the type-IIB* algebra with R-symmetry group $\mathrm{O}(1,1)$. Similarly, the type-IIA* algebra, $\epsilon=-1$, is mapped to the type-IIB* algebra by spacelike T-duality and to the type-IIB algebra under timelike T-duality. In this convention it is manifest that the four real algebras are different real forms of two complex algebras, because we have imposed that the complex superbrackets assume a standard form, that is $M_{i j}=\delta_{i j}$.

2. We can choose $\beta$ such that the reality conditions is a standard Majorana condition, $L_{i j}=\delta_{i j}$, which requires $\beta^{2}=\epsilon$. In this case the real supersymmetry algebras are distinguished by their bilinear form, and for the IIB* theories the bilinear form is twisted by a relative minus sign:

$$
\left(M_{i j}\right)=\left(\begin{array}{cc}
1 & 0 \\
0 & \pm \epsilon
\end{array}\right), \quad\left(L_{i j}\right)=\left(\begin{array}{ll}
1 & 0 \\
0 & 1
\end{array}\right)
$$

This convention was used when the type-IIB* algebra was originally introduced as a twisted version of the IIB algebra [1].

\section{2 $\mathcal{N}=2$ supersymmetry in general ten-dimensional signatures}

By inspection of table 8 , there are three types of signature for ten-dimensional $\mathcal{N}=2$ algebras.

- In signatures $(1,9),(5,5)$ and $(9,1)$ there are three possible R-symmetry groups $\mathbb{Z}_{2} \times \mathbb{Z}_{2}, \mathrm{O}(2), \mathrm{O}(1,1)$. These are orthogonal Weyl-compatible signatures with two real structures on $\mathbb{S}$. We have discussed the case $(1,9)$ before. Signature $(9,1)$ can be viewed as signature $(1,9)$ with a mostly minus convention for the metric. ${ }^{27}$ Our classification shows that in neutral signature $(5,5)$ algebras of type IIA/IIA*/IIB/IIB* exist and are related by T-duality. The existence of the corresponding string theories is known from [2].

\footnotetext{
${ }^{27}$ While the Clifford algebras are not the same, the resulting spin representations are isomorphic and we have not found any indication that signatures $(1,9)$ and $(9,1)$ give rise to different physics.
} 
- In signature $(0,10),(2,8),(4,6),(6,4),(8,2),(10,0)$ the unique R-symmetry group is $\mathbb{Z}_{2}$. These are orthogonal Weyl incompatible signatures which only admit $(1,1)$ algebras. The existence of type-IIA string theories in these signatures was established in [2].

- In signature $(3,7)$ and $(7,3)$ the unique R-symmetry group is $\mathrm{SO}(2)$. These are orthogonal, Weyl-compatible signatures with two quaternionic structures on $\mathbb{S}$. This implies that the only $\mathcal{N}=2$ algebras are chiral $(2,0)$ (or $(0,2)$ ) algebras. The existence of type-IIB string theories in these signatures was established in [2].

In summary, the network of ten-dimensional type-II string theories described in [2] exhausts all ten-dimensional $\mathcal{N}=2$ supersymmetry algebras. To relate type-II string theories across signatures, one needs to combine spacelike and timelike T-duality with S-duality. T-duality changes the space-time signature if the string world-sheet has Euclidean signature. S-duality is needed to map type-IIB* string theory to type-IIB' string theory. This exchanges the fundamental string with the E2-brane, thus providing a fundamental string with Euclidean world-sheet. Since we only consider the supersymmetry algebras, which do not distinguish between type-IIB* and type-IIB', we can establish the relation between ten-dimensional $\mathcal{N}=2$ algebras in different signatures by relating them to the same nine-dimensional $\mathcal{N}=2$ algebra.

\section{3 $\mathcal{N}=2$ supersymmetry in nine dimensions}

'Pure', that is spacelike and timelike T-dualities, arise whenever after compactification over space or over time, the limit $R \rightarrow 0$ corresponds to an alternative decompactification limit $R^{\prime}=1 / R \rightarrow \infty$. At the level of the supersymmetry algebras this requires that the two ten-dimensional supersymmetry algebras give rise, by compactification, to the same nine-dimensional supersymmetry algebra. Since ten-dimensional theories in signatures $(t, s)$ and $(t-1, s+1)$ can reduce to the same theory in signature $(t-1, s)$ by applying timelike and spacelike reduction, respectively, this opens up the possibility of 'mixed' T-dualities, where one reduces over time and decompactifies over space, or vice versa. Our formalism gives a uniform description of superymmetry algebras across dimensions and signatures, and allows to work out universal formulae for the spacelike and timelike reduction of these algebras. We have collected the relevant formulae in appendix F. Using this machinery it is straightforward to work out which of the ten-dimensional $\mathcal{N}=2$ algebras reduce to the same nine-dimensional algebra, and are thus related by a pure (signature preserving) or mixed (signature changing) T-duality.

\subsubsection{Nine-dimensional $\mathcal{N}=2$ supersymmetry algebras}

We start by surveying nine-dimensional $\mathcal{N}=2$ supersymmetry algebras. In nine dimensions the Majorana bilinear form on $\mathbb{S}$ is super-admissible, so we use a symmetric bilinear form on $\mathbb{C}^{K}$, thus leading to R-symmetry groups that are real forms of $\mathrm{O}(K, \mathbb{C})$. The two Majorana bilinears in ten dimensions are based on the two ten-dimensional charge conjugation matrices $C_{ \pm}^{(10)}$, which have invariants $(\sigma, \tau)=(\mp 1, \mp 1)$. The nine-dimensional charge conjugation matrix $C^{(9)}$ has invariants $(\sigma, \tau)=(+1,+1)$. In $10 \mathrm{D}$ we will use $C_{-}^{(10)}$ in this section as this turns out to be convenient, and we know that both choices define the same real 


\begin{tabular}{|c|c|c|c|c|c|}
\hline Signature & $(0,9)$ & $(1,8)$ & $(2,7)$ & $(3,6)$ & $(4,5)$ \\
\hline R-sym. Group & $\mathrm{O}(1,1)$ or $\mathrm{O}(2)$ & $\mathrm{O}(1,1)$ or $\mathrm{O}(2)$ & $\mathrm{SO}(2)$ & $\mathrm{SO}(2)$ & $\mathrm{O}(1,1)$ or $\mathrm{O}(2)$ \\
\hline
\end{tabular}

Table 11. $\mathcal{N}=2$ R-symmetry groups in nine dimensions, the R-symmetry group for $(s, t)$ is the same as $(t, s)$.

supersymmetry algebra. The nine-dimensional $\mathcal{N}=2$ algebras and their R-symmetry groups are listed in table 11.

There are two types of nine-dimensional signatures.

- In signatures $(0,9),(1,8),(4,5)$, and those signatures obtained by flipping $t \leftrightarrow s$, the complex spinor module $\mathbb{S}$ has an invariant real structure, which allows one to impose a standard Majorana condition with R-symmetry group $\mathrm{O}(2)$ or a twisted Majorana condition with R-symmetry group $\mathrm{O}(1,1)$.

- In signatures $(2,7),(3,6)$ and those signatures obtained by flipping $t \leftrightarrow s$, the complex spinor module $\mathbb{S}$ carries an invariant quaternionic structure, which allows one to impose a symplectic Majorana condition. In this case the R-symmetry group is $\mathrm{SO}(2)$.

Explicitly, the reality conditions are:

$$
\begin{aligned}
& \left(\lambda^{i}\right)^{*}=\alpha B^{(t, s)} \lambda^{i} \quad \Longrightarrow G_{R}=\mathrm{O}(2), \\
& \left(\lambda^{i}\right)^{*}=\alpha B^{(t, s)} \lambda^{j} \eta_{j i} \Longrightarrow G_{R}=\mathrm{O}(1,1), \\
& \left(\lambda^{i}\right)^{*}=\alpha B^{(t, s)} \lambda^{j} \epsilon_{j i} \Longrightarrow G_{R}=\mathrm{SO}(2) .
\end{aligned}
$$

\subsubsection{Reduction of Clifford algebras}

Next, we give the explicit relations between ten- and nine-dimensional quantities. We suppress most details, which are straightforward to work out using appendix F. The basis for the ten-dimensional Clifford algebra is $\Gamma_{\mu}$ within which we embed the nine-dimensional gamma matrices, $\gamma_{\mu}$, according to

$$
\Gamma_{\mu}=\gamma_{\mu} \otimes \sigma_{1}, \quad \Gamma_{10}=\mathrm{Id} \otimes \sigma_{2} \quad \text { or } \quad \Gamma_{0}=i \mathrm{Id} \otimes \sigma_{2} .
$$

The Majorana bilinear forms on the respective complex spinor modules are related by

$$
C_{-}^{(10)}=C^{(9)} \otimes \sigma_{1}
$$

The 10D chiral projection matrix $\Gamma_{*}=(-i)^{t+\frac{D}{2}} \Gamma_{1} \ldots \Gamma_{10}$ decomposes as

$$
\Gamma_{*}=1 \otimes \sigma_{3} .
$$

In non-chiral type-IIA theories we have two spinors of opposite chirality, denoted $\lambda_{ \pm}$, which decompose into nine-dimensional spinors $\psi^{1}$ and $\psi^{2}$ as

$$
\begin{aligned}
& \lambda_{+}=\psi^{1} \otimes\left(\begin{array}{l}
1 \\
0
\end{array}\right), \\
& \lambda_{-}=\psi^{2} \otimes\left(\begin{array}{l}
0 \\
1
\end{array}\right) .
\end{aligned}
$$


In chiral type-IIB theories we have two spinors of the same chirality, $\lambda_{+}^{1}$ and $\lambda_{+}^{2}$, which decompose into nine dimensional spinors, again denoted $\psi^{1}$ and $\psi^{2}$, as

$$
\begin{aligned}
& \lambda_{+}^{1}=\psi^{1} \otimes\left(\begin{array}{l}
1 \\
0
\end{array}\right), \\
& \lambda_{+}^{2}=\psi^{2} \otimes\left(\begin{array}{l}
1 \\
0
\end{array}\right) .
\end{aligned}
$$

\subsubsection{Reduction of reality conditions}

Next, we dimensionally reduce ten-dimensional reality conditions. For simplicity and without loss of generality we will only consider $B_{-}^{(p, q)}$ (with $p+q=10$ ). A spacelike reduction gives

$$
B_{-}^{(t, s+1)}=B^{(t, s)} \otimes \sigma_{1}^{t+1}= \begin{cases}B^{(t, s)} \otimes \sigma_{1} & \text { for } t \text { even, } \\ B^{(t, s)} \otimes 1 & \text { for } t \text { odd }\end{cases}
$$

while a timelike reduction gives

$$
B_{-}^{(t+1, s)}=B^{(t, s)} \otimes i \sigma_{3} \sigma_{1}^{t}= \begin{cases}i B^{(t, s)} \otimes \sigma_{3} & \text { for } t \text { even } \\ -B^{(t, s)} \otimes \sigma_{2} & \text { for } t \text { odd }\end{cases}
$$

The second factor in the tensor products captures the Weyl-compatibility of the tendimensional signature; we observe that when the ten-dimensional signature has an even number of timelike directions we have a factor $\sigma_{1}$ or $\sigma_{2}$ that exchanges chiralities, as in these signatures the reality condition is Weyl-incompatible. When the ten-dimensional theory has an odd number of timelike directions the reality condition is Weyl-compatible, so we get Id or $\sigma_{3}$ which do not mix the two chiralities.

As an example we reduce the type-IIA algebra in signature $(0,10)$ (this is the unique $\left(\mathcal{N}_{+}, \mathcal{N}_{-}\right)=(1,1)$ algebra in this signature $)$ to $(0,9)$. The $(0,10)$ parent theory involves a single Majorana spinor that can be written in terms of a Weyl-incompatible reality condition as

$$
\left(\lambda_{ \pm}\right)^{*}=\alpha B_{-}^{(0,10)} \lambda_{\mp} .
$$

Decomposing into nine-dimensional quantities, we see this reads

$$
\begin{aligned}
& \left(\psi^{1}\right)^{*} \otimes\left(\begin{array}{l}
1 \\
0
\end{array}\right)=\alpha\left(B^{(0,9)} \otimes \sigma_{1}\right)\left(\psi^{2} \otimes\left(\begin{array}{l}
0 \\
1
\end{array}\right)\right)=\alpha B^{(0,9)} \psi^{2} \otimes\left(\begin{array}{l}
1 \\
0
\end{array}\right), \\
& \left(\psi^{2}\right)^{*} \otimes\left(\begin{array}{l}
0 \\
1
\end{array}\right)=\alpha\left(B^{(0,9)} \otimes \sigma_{1}\right)\left(\psi^{1} \otimes\left(\begin{array}{l}
1 \\
0
\end{array}\right)\right)=\alpha B^{(0,9)} \psi^{1} \otimes\left(\begin{array}{l}
1 \\
0
\end{array}\right) .
\end{aligned}
$$

Dropping the vector $\left(\begin{array}{l}1 \\ 0\end{array}\right)$ we can simply write

$$
\left(\psi^{i}\right)^{*}=\alpha B^{(0,9)} \psi^{j} \eta_{j i}
$$

This leads to a $(0,9) \mathcal{N}=2$ superalgebra with an $\mathrm{O}(1,1)$ R-symmetry group. 


\subsubsection{Reduction of vector-valued bilinear forms}

Next, we need to reduce the vector-valued bilinear form. A non-chiral, type-IIA vector-valued bilinear form reduces as

$$
\left(\Gamma^{\mu} \lambda_{+}\right)^{T} C_{-}^{(10)} \chi_{+}+\left(\Gamma^{\mu} \lambda_{-}\right)^{T} C_{-}^{(10)} \chi_{-}=\left(\gamma^{\mu} \psi^{i}\right)^{T} C^{(9)} \phi^{j} \delta_{j i} \otimes 1-i\left(\psi^{i}\right)^{T} C^{(9)} \phi^{j} \eta_{j i} \otimes 1 .
$$

The second term is a scalar under the nine-dimensional Poincare Lie algebra and therefore gives rise to a central extension of the nine-dimensional supersymmetry algebra. This term is only relevant for states which have momentum along the direction we reduce over, as this momentum component corresponds to the central charge. We remark that the ten-dimensional algebra also admits BPS extensions (poly-vector extensions) corresponding to terms on the r.h.s. which transform as antisymmetric tensors under the Lorentz group. While such terms are important since they encode the possible BPS branes of a theory, we have decided to leave the inclusion of BPS extensions to future work. Neglecting the central term, non-chiral, type IIA vector-valued forms therefore reduce as

$$
\left(\Gamma^{\mu} \lambda_{+}\right)^{T} C_{-}^{(10)} \chi_{+}+\left(\Gamma^{\mu} \lambda_{-}\right)^{T} C_{-}^{(10)} \chi_{-} \rightarrow\left(\gamma^{\mu} \psi^{i}\right)^{T} C^{(9)} \phi^{j} \delta_{j i} .
$$

Similarly, chiral, type IIB vector valued forms reduce as

$$
\left(\Gamma^{\mu} \lambda_{+}^{i}\right)^{T} C_{-}^{(10)} \chi_{+}^{j} \delta_{j i} \rightarrow\left(\gamma^{\mu} \psi^{i}\right)^{T} C^{(9)} \phi^{j} \delta_{j i}
$$

\subsubsection{Summary of relations between ten-dimensional and nine-dimensional $\mathcal{N}=2$ supersymmetry algebras}

We will not include further details of computations, as the principle is by now clear and the various signatures only differ by sign factors. The results are summarized in the diagram in table 12. This diagram shows in particular that by a sequence of timelike reductions and spacelike oxidations, that is by mixed T-dualities, one can connect all signatures to one another. While in generic ten-dimensional signatures there is a unique $\mathcal{N}=2$ algebra which is chiral or non-chiral, there are two chiral and two non-chiral algebras in Lorentz and in neutral signature, which then are mutually related by pure T-dualities.

\subsection{Four-dimensional $\mathcal{N}=2$ supersymmetry algebras and type-II/II* Calabi-Yau compactifications}

The compactification of type-IIA/IIB string theory in signature $(1,9)$ on a Calabi-Yau three-fold with Hodge numbers $h^{1,1}, h^{2,1}$ leads to an $\mathcal{N}=2$ supergravity theory in signature $(1,3)$ with $n_{V}$ vector multiplets and $n_{H}$ hypermultiplets, where $\left(n_{V}, n_{H}\right)=\left(h^{1,1}, h^{2,1}+1\right)$ for type-IIA and $\left(n_{V}, n_{H}\right)=\left(h^{2,1}, h^{1,1}+1\right)$ for type-IIB [28-30]. The four-dimensional supersymmetry algebra is the standard $\mathcal{N}=2$ supersymmetry algebra with R-symmetry group $G_{R}=\mathrm{U}(2) \cong \mathrm{U}(1) \times \mathrm{SU}(2)$. The target space geometries of vector and hypermultiplets are projective special Kähler and quaternionic-Kähler, see [31] for a review. As already shown in [17], there is a second, inequivalent $\mathcal{N}=2$ algebra in signature $(1,3)$, where the supercharges obey a twisted Majorana condition and the R-symmetry group is $\mathrm{U}(1,1) \cong$ $\mathrm{U}(1) \times \mathrm{SU}(1,1)$. The corresponding theory of rigid vector multiplets was constructed 


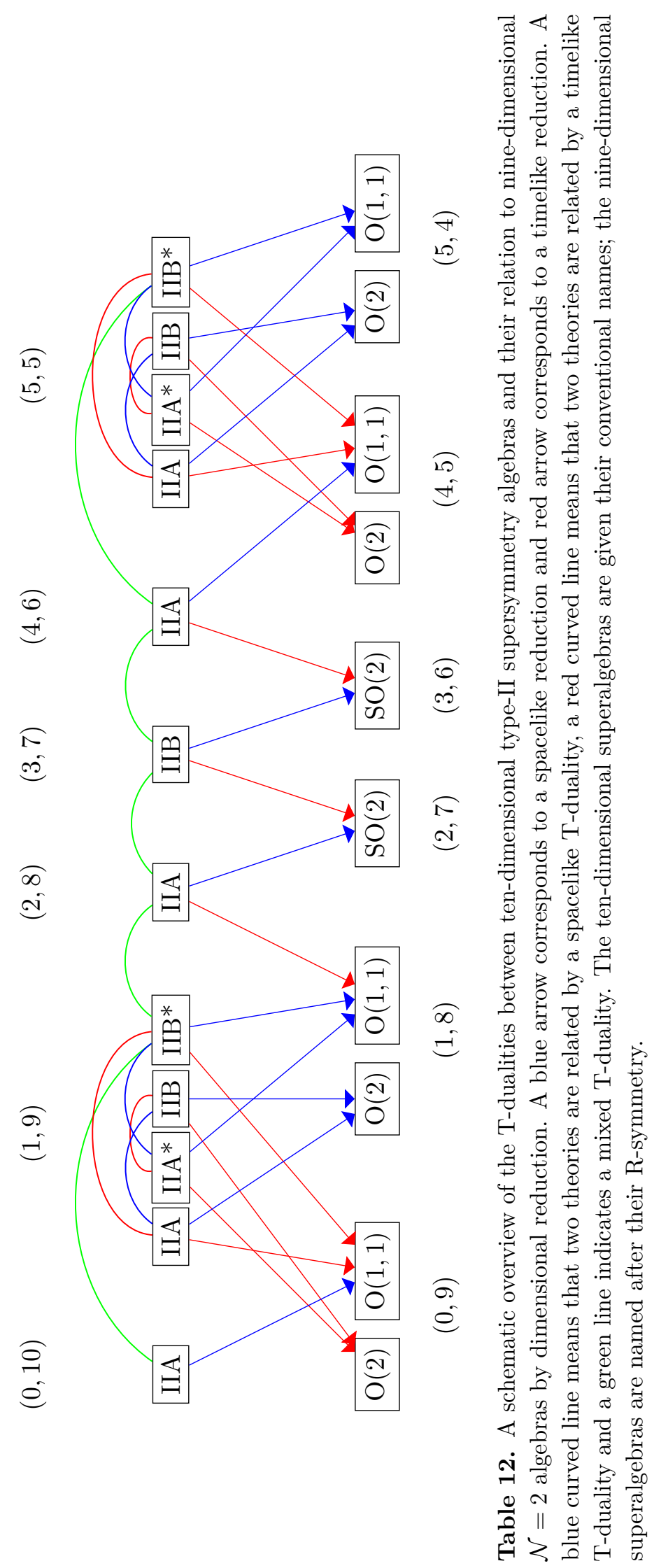


explicitly in [17], where it was shown that while the scalar geometry of the vector multiplets is still special Kähler, the modified supersymmetry transformations imply a relative sign between the scalar and vector kinetic terms. While hypermultiplets for the supersymmetry with $\mathrm{U}(1,1)$ R-symmetry have not yet been constructed, sign flips between the kinetic terms of the hypermultiplet scalars are expected, and the factor $\mathrm{SU}(1,1)$ suggests that the scalar geometry is para-quaternionic Kähler rather than quaternionic Kähler. The reason is as follow: for the standard algebra with R-symmetry $\mathrm{U}(2) \cong \mathrm{U}(1) \times \mathrm{SU}(2)$ the vector multiplet and hypermultiplet scalars transform under $\mathrm{U}(1)$ and $\mathrm{SU}(2)$, respectively. The factor $\mathrm{U}(1)$ acts on the vector multiplet scalar manifold as multiplication by the complex structure, that is, as 'multiplication by $i$ '. Similarly, the factor $\mathrm{SU}(2)$ acts on the hypermultiplet scalar manifold as multiplication by the unit quaternions $\mathrm{SU}(2) \subset \mathbb{H}^{*}$. A change of the R-symmetry group therefore indicates a change of the scalar geometry. For example, in signature $(0,4)$ the R-symmetry group is $\mathrm{U}^{*}(2) \cong \mathrm{SO}(1,1) \times \mathrm{SU}(2)$, and the geometry of the vector multiplet scalars is para-Kähler, which differs from Kähler by replacing the complex structure $I, I^{2}=-\mathrm{Id}$ by a para-complex structure $E \neq \mathrm{Id}, E^{2}=\mathrm{Id}$. The action of the factor $\mathrm{SO}(1,1) \subset \mathrm{U}^{*}(2)$ on the vector multiplet scalar manifold is generated by the action of the para-complex structure, see [12] for details. Similarly, the replacement of the factor $\mathrm{SU}(2) \subset \mathrm{U}(2)$ by $\mathrm{SU}(1,1) \subset \mathrm{U}(1,1)$ indicates that the scalar geometry of hypermultiplets in the twisted $\mathcal{N}=2$ theory in signature $(1,3)$ is para-hyper-Kähler for rigid supersymmetry and para-quaternion-Kähler for local supersymmetry. By inspection of the four-dimensional R-symmetry groups we can determine the scalar geometries of vector and hypermultiplets for all signatures and inequivalent algebras, see table 13 .

For some of these cases the scalar geometry has been verified by explicit construction, while other cases will be the subject of future work. $\mathcal{N}=2$ supergravity in signature $(0,4)$ arises from the compactification of the Euclidean (signature $(0,10)$ ) type-IIA theory on a Calabi-Yau threefold. The R-symmetry group $\mathrm{U}^{*}(2) \cong \mathrm{SO}(1,1) \times \mathrm{SU}(2)$ indicates that the vector multiplet geometry is special para-Kähler while the hypermultiplet geometry is quaternionic-Kähler, which was indeed found in [10] by explicit dimensional reduction. In [32] it will be shown that theories realizing the twisted $\mathcal{N}=2$ algebra in signature $(1,3)$ arise from the compactification of type-IIA*/IIB* string theory on Calabi-Yau three-folds, and that their hypermultiplet geometry is indeed para-quaternion Kähler. $\mathcal{N}=2$ supergravity in signature $(2,2)$ arises from the compactification of the signature $(2,8)$ type-IIA theory on a Calabi-Yau threefold. The R-symmetry group $\mathrm{GL}(2, \mathbb{R}) \cong \mathbb{R}^{>0} \times \mathrm{SL}^{ \pm}(2, \mathbb{R})$ indicates that the vector multiplet geometry is special para-Kähler while the hypermultiplet geometry is para-quaternionic-Kähler. ${ }^{28}$ This will be verified by dimensional reduction in [32].

\subsection{Three-dimensional $\mathcal{N}=4$ supersymmetry algebras and their hypermultiplet geometries}

Three-dimensional $\mathcal{N}=4$ supergravity theories can be realized by spacelike and timelike reductions of four-dimensional $\mathcal{N}=2$ supergravity. In three dimensions vector multiplets can be dualized into hypermultiplets so that the scalar manifold is the product of two

\footnotetext{
${ }^{28} \mathrm{SL}^{ \pm}(2, \mathbb{R}) \subset \mathrm{GL}(2, \mathrm{R})$ is the group of real $2 \times 2$ matrices with determinant \pm 1 .
} 


\begin{tabular}{|l|l|l|l|}
\hline Signature & R-symmetry & VM geometry & HM geometry \\
\hline$(0,4)$ & $\mathrm{U}(2)^{*} \cong \mathrm{SO}(1,1) \times \mathrm{SU}(2)$ & SPK & QK \\
\hline \multirow{2}{*}{$(1,3)$} & $\mathrm{U}(2) \cong \mathrm{U}(1) \times \mathrm{SU}(2)$ & SK & QK \\
\cline { 2 - 4 } & $\mathrm{U}(1,1) \cong \mathrm{U}(1) \times \mathrm{SU}(1,1)$ & SK & PQK \\
\hline$(2,2)$ & $\mathrm{GL}(2, \mathbb{R}) \cong \mathrm{SO}(1,1) \times \mathrm{SL}^{ \pm}(2, \mathbb{R})$ & SPK & PQK \\
\hline
\end{tabular}

Table 13. Four-dimensional $\mathcal{N}=2$ supersymmetry algebras, their R-symmetry groups and the scalar geometries of vector multiplets (VM) and hypermultiplets (HM). SK = special Kähler, SPK = special para-Kähler, QK = quaternionic Kähler, PQK = para-quaternionic Kähler.

\begin{tabular}{|l|l|l|l|}
\hline Signature & R-symmetry & $\mathrm{HM}_{1}$ geometry & $\mathrm{HM}_{2}$ geometry \\
\hline \multirow{2}{*}{$(0,3)$} & $\mathrm{SO}^{*}(4) \cong \mathrm{SL}(2, \mathbb{R}) \times \mathrm{SU}(2)$ & $\mathrm{PQK}$ & $\mathrm{QK}$ \\
\hline & $\mathrm{O}(4) \cong \mathrm{SU}(2) \times \mathrm{SU}(2)$ & $\mathrm{QK}$ & $\mathrm{QK}$ \\
\cline { 2 - 4 } & $\mathrm{O}(1,3)$ & - & - \\
\cline { 2 - 4 } & $\mathrm{O}(2,2) \cong \mathrm{SL}(2, \mathbb{R}) \times \mathrm{SL}(2, \mathbb{R})$ & $\mathrm{PQK}$ & $\mathrm{PQK}$ \\
\hline
\end{tabular}

Table 14. Three-dimensional $\mathcal{N}=4$ supersymmetry algebras, their R-symmetry groups and the scalar geometries of the two hypermultiplet manifolds.

hypermultiplet manifolds. The R-symmetry groups in three dimensions are $\mathrm{SO}^{*}(4) \cong$ $\mathrm{SL}(2, \mathbb{R}) \times \mathrm{SU}(2)$ in Euclidean signature $(0,3)$ and $\mathrm{O}(p, q), p+q=4$ in Lorentz signature $(1,2)$. For all cases with the exception of $\mathrm{O}(1,3)$ this allows us to identify the hypermultiplet geometries, see table 14 . The case $\mathrm{O}(1,3)$ is special in that it is the only one which does not arise by dimensional reduction. For the other cases we note the embeddings $\mathrm{U}^{*}(2) \subset \mathrm{SO}^{*}(4)$, $\mathrm{U}(2) \subset \mathrm{O}(4)$, and $\mathrm{U}(1,1) \subset \mathrm{O}(2,2)$ of the respective R-symmetry groups.

For some of these cases the scalar geometry has been verified by explicit construction, while others will be subject to future work. Dimensional reduction of vector multiplets from signatures $(0,4)$ or $(1,3)$ to signature $(0,3)$ leads to hypermultiplets with a para-quaternionic Kähler target space [15]. By comparing to table 12 we see that the resulting pattern of spacelike and timelike reductions replicates the one for ten- and nine-dimensional type-II theories, with the exception that in signature $(1,3)$ there are only two rather than four non-isomorphic supersymmetry algebras. However, compactifications of type-IIA and typeIIB on the same Calabi-Yau threefolds lead to different theories since, as reviewed above, the roles of vector and hypermultiplets are reversed. The resulting pattern of dimensional reductions and oxidations, and the induced T-dualities between the four-dimensional theories will be studied in detail in [32].

\section{Conclusion and outlook}

In this paper we have provided a construction of extended supersymmetry algebras which works uniformly across dimensions and signatures. We have classified the resulting Rsymmetry groups, which ultimately leads to table 10 . The resulting pattern of R-symmetry 
groups is modulated by the properties of the complex bilinear form and of the reality conditions. In some signatures multiple real forms lead to several non-isomorphic algebras based on the same spinor module. This includes 'type-*' Lorentzian signature algebras with non-compact R-symmetry groups such as $\mathrm{O}(p, q)$ in signatures $(1,9)$ and $(1,2)$, and $\mathrm{U}(k, l)$ in signature $(1,3)$ which correspond to non-standard, 'twisted' Majorana conditions imposed on complex supercharges. Our formalism always starts with a standard complex superbracket, so that the 'twisting' is encoded in the reality condition. This has the advantage that the twisting is tied to selecting a real form of the complex R-symmetry group, which allows us to see 'twisting' as part of the larger pattern of variation of R-symmetry group across dimensions and signatures.

While we have provided evidence that supersymmetry algebras are classified by their R-symmetry groups, together with the choice of one relative sign for chiral algebras, solving the classification problem completely has been left to future work. The most promising approach is to combine the formalism applied in this paper with the methods and results of [22], as has been done for the special case of $D=4, \mathcal{N}=2$ supersymmetry in [17]. BPS-charges can be included along the lines of [24], which will also allow to discuss the tensor-valued bilinear forms needed to describe fermionic terms in the action $[2,16]$. Our formalism, which encodes the signature dependence completely in the reality condition, while the superbracket is fixed in a given dimension allows to obtain the supersymmetry transformations and actions in a given signature by imposing reality conditions on their complex versions [16].

Apart from ab initio construction, dimensional reduction can be used to obtain supersymmetry transformations and actions. In section 8 we have discussed Calabi-Yau compactifications of type-II superstring theories to theories in signatures $(0,4),(1,3)$ and $(2,2)$ using their supersymmetry algebras and R-symmetry groups. The corresponding bosonic actions which will be constructed explicitly in [32]. This will not only allow us to verify the claims we have made regarding the target space geometries of four- and three-dimensional hypermultiplets in arbitrary signature, but also prepare the ground for studying solutions of 'exotic' supergravity and string theories in dimension 4 and 10. It was observed in [33] that there is a correspondence between the planar cosmological solutions of standard $D=4, \mathcal{N}=2$ supergravity with vector multiplets constructed in [34], and planar black hole solutions of its twisted variant (with R-symmetry $\mathrm{U}(1,1)$ ). As these include solutions of the STU-model (and its twisted version), they can be lifted to 10 and 11 dimensions to solutions of type-II string theory and M-theory. In fact, in the untwisted case, this leads to the same higher-dimensional brane configuration as the STU black hole [34]. The solutions of the twisted four-dimensional theory lift to brane configurations in type-II* theories, which strongly suggests that the duality between black hole and cosmological solutions can be understood as a combination of time-like and space-like T-dualities. As shown in [33] the horizons of the dual cosmological/black hole solutions satisfy the first law of thermodynamics and have the same semi-classical Euclidean partition function. Investigating this from the ten-dimensional point of view is likely to provide new insights into the solutions of type-II* theories. This is but one example how one can further explore the network of type-II string theories described in [1, 2]. The work of $[3,4]$ shows that there 
are many field-theoretical as well as phenomenological aspects to be explored, including signature change, de Sitter solutions, and brane world scenarios.

Another direction to explore is what can be learned about the symmetries underlying string theories. Dimensional reduction exhibits hidden symmetry, and maximal symmetry is reached when all directions, including time, are compactified [35]. However, if string theories in general signature are part of the full configuration space of string/M-theory, then dimensional reduction is not enough to exhibit the full symmetry of string theory. In order to cover all possible spacetime signatures one can use complexification followed by exploring all possible reality conditions. For example in $[36,37]$ a uniform description of $M$-theory and type-II theory was given based on the complex form of the ortho-symplectic Lie superalgebra $\mathfrak{o} \mathfrak{s p}(32)$. The formalism presented in this paper may offer certain advantages, because it does not rely on obtaining Poincaré Lie superalgebras as contractions, but instead works directly with complex and real Poincaré Lie superalgebras. Another strategy to explore the hidden symmetries of string theory is to use an extended spacetime, as is done in doubled and exceptional field theory. In these formalisms exotic versions of type-II string theory appear naturally, see for example [38], and the formalism presented in this paper should be useful to investigate this further.

\section{Acknowledgments}

This paper is substantially based on parts of the first authors' $\mathrm{PhD}$ thesis [39], which is available at the University of Liverpools 'Elements' repository. T.M. thanks Vicente Cortés for useful discussions.

\section{A Conventions, notation and some useful formulae}

In this appendix we collect information about our notations and conventions. In particular, we explain our conventions for spinor indices, which we suppress where possible in the paper. We also explain in detail how to relate the formulation of supersymmetry algebras in terms of vector-valued bilinear forms to the usual one in terms of anti-commutators of supercharges.

\section{A.1 Spinor index conventions}

For spinor indices we use the same conventions as [12]. Dirac spinors $\psi \in \mathbb{S}$ have lower indices, $\psi=\left(\psi_{\alpha}\right)$. Since $\gamma$-matrices represent endomorphisms on the spinor module $\mathbb{S}$, their index structure is $\gamma_{\mu}=\left(\gamma_{\mu \alpha}^{\beta}\right)$.

The matrices $A$ and $C$ relate the $\gamma$-matrices to their Hermitian conjugate and to the transposed matrices, respectively (2.4), (2.6). They have two upper spinor indices, $A=\left(A^{\alpha \beta}\right)$ and $C=\left(C^{\alpha \beta}\right)$, and define a sesquilinear form (the Dirac bilinear form) and a complex bilinear form (the Majorana bilinear form) on $\mathbb{S}$, respectively:

$$
A(\lambda, \chi)=\lambda_{\alpha}^{*} A^{\alpha \beta} \chi_{\beta}, \quad C(\lambda, \chi)=\lambda_{\alpha} C^{\alpha \beta} \chi_{\beta} .
$$

The inverse matrices are $A^{-1}=\left(A_{\alpha \beta}^{-1}\right)$ and $C^{-1}=\left(C_{\alpha \beta}^{-1}\right)$. 
When using Dirac spinors with a sesquilinear form $A$, spinor indices are raised and lowered using $A$ and $A^{-1}$, i.e. $\lambda^{\alpha}=A^{\alpha \beta} \lambda_{\beta}$ and $\lambda_{\alpha}=A_{\alpha \beta}^{-1} \lambda^{\beta}$. Upper index spinors defined this way are elements of complex-conjugate dual (transposed) Dirac spinor module, which we can identify with $\mathbb{S}$ using the spin equivariant isomorphism provided by $A$. Similarly, we can raise and lower Dirac spinor indices using $C$ and its inverse: $\lambda^{\alpha}=C^{\alpha \beta} \lambda_{\beta}$ and $\lambda_{\alpha}=C_{\alpha \beta}^{-1} \lambda^{\beta}$. If upper index spinors are defined this way, they are elements of the dual (transposed) Dirac spinor module, which we can identify with $\mathbb{S}$ using the spin equivariant isomorphism provided by $C$. In this paper we exclusively use $C$ and $C^{-1}$ to raise and lower Dirac spinor indices. We use a convention where the matrix $C$ is equal to its inverse, so that we can denote the components of $C^{-1}$ simply by $C_{\alpha \beta}$. The matrix $C$ is either symmetric or antisymmetric. This is encoded by the invariant $\sigma=\sigma_{C}$, which also is the symmetry of the Majorana bilinear form,

$$
C^{\beta \alpha}=\sigma C^{\alpha \beta} .
$$

In the antisymmetric case some care is required when raising and lowering indices. For example

$$
C^{\alpha \beta} C_{\alpha \gamma} C_{\beta \delta}=\sigma C_{\gamma \alpha} C^{\alpha \beta} C_{\beta \delta}=\sigma C_{\gamma \delta} .
$$

Note that the convention we use for spinor indices is not of the 'NW-SE type.' This is different from our convention for 'internal' indices on spinors, see below. In the bulk of this paper we work with expressions where spinor indices are contracted and can be omitted. However, index conventions are relevant when comparing our results to the literature. In particular, they are relevant for translating between vector-valued forms and anti-commutators of supercharges, see below.

Equations like $A=\Pi_{\tau} \gamma_{\tau}$ (where $\tau$ runs over all timelike Lorentz indices), which equate the matrix of a bilinear form (two upper indices) to a matrix representing an endomorphism (one lower, one upper index) are equations between matrices, not between maps. Put differently, once we have fixed a set of $\gamma$-matrices, we can make a choice for the matrix $A$, but this choice is tied to our choice of $\gamma$-matrices.

\section{A.2 Index conventions for internal indices}

For indices on the auxiliary space $\mathbb{C}^{K}$ we will usually ${ }^{29}$ use the NW-SE convention. This reflects that our formalism generalizes symplectic Majorana spinors, where the R-symmetry group $\mathrm{SU}(2)$ acts on these indices. Adopting this as the universal convention, bilinear forms on $\mathbb{C}^{K}$ take the form

$$
M(z, w)=z^{i} w^{j} M_{j i}, \quad i, j=1, \ldots, K
$$

Raising and lowering the indices is done using $M_{i j}$ and its contragradient (transposed of inverse) $M^{i j}$ such that

$$
z^{i}=M^{i j} z_{j}, \quad z_{i}=z^{j} M_{j i}
$$

\footnotetext{
${ }^{29}$ The only exception is the 'matrix notation' which we use when doubling the dimension of the auxiliary space to completely disentangle the spin and R-symmetry groups for even-dimensional space-times, see appendix D.
} 
Note that the NW-SE convention then implies

$$
M^{i j} M_{k j}=\delta_{k}^{i},
$$

so that for anti-symmetric bilinear forms the matrices $\left(M^{i j}\right)$ and $\left(M_{i j}\right)$ are not each others inverse, but the inverse multiplied by -1 .

Our construction requires the choice of a non-degenerate complex bilinear form, which is either symmetric or antisymmetric. Our preferred choices are the symmetric bilinear form $\delta$ and antisymmetric bilinear form $J$, with Gram matrices

$$
\delta=\mathbb{1}_{N}, \quad J=\left(\begin{array}{cc}
0 & \mathbb{1}_{\frac{K}{2}} \\
-\mathbb{1}_{\frac{K}{2}} & 0
\end{array}\right) .
$$

We refer to these bilinears and matrices as 'canonical.'

\section{A.3 Poincaré Lie superalgebras with and without indices}

In this section we explain how the standard, index-based notation for supersymmetry is related to the index-free description (or sometimes only partly index-free description) used in [22] and in this paper.

A Poincaré Lie superalgebra, $\mathfrak{g}$, is a $\mathbb{Z}_{2}$-graded Lie algebra

$$
\mathfrak{g}=\mathfrak{g}_{0}+\mathfrak{g}_{1}, \quad \mathfrak{g}_{0}=\mathfrak{s o}(V)+V,
$$

where the even part $\mathfrak{g}_{0}$ is the Poincaré Lie algebra based on the vector space $V=\mathbb{R}^{t, s}$, that is $\mathbb{R}^{t+s}$ equipped with a real symmetric bilinear form with signature $(t, s)$. The Lie bracket on $\mathfrak{g}_{0}$ is

$$
[A, B]=A B-B A, \quad[A, v]=A v, \quad\left[v_{1}, v_{2}\right]=0,
$$

where $A, B \in \mathfrak{s o}(V)$ and $v, v_{1}, v_{2} \in V$.

The odd part $\mathfrak{g}_{1}$ of $\mathfrak{g}$ is an arbitrary sum of irreducible spinor modules, that is of $\mathfrak{s p i n}(V) \cong \mathfrak{s o}(V)$ modules obtained by decomposing irreducible modules of the real Clifford algebra $C l(V) \cong C l_{t, s}$ into irreducible modules of $\mathfrak{s p i n}(V) \subset C l_{t, s}$. The Lie algebra structure on $\mathfrak{g}_{0}$ is extended to a Lie superalgebra structure by the spinorial action $\rho_{S}$ of $\mathfrak{s o}(V)$ on $\mathfrak{g}_{1}$ together with a trivial action of $V$ on $\mathfrak{g}_{1}$, and a symmetric vector-valued bracket $\mathfrak{g}_{1} \times \mathfrak{g}_{1} \rightarrow V$, which is $\mathfrak{s o}(V)$ equivariant (covariant). The additional non-trivial relations are:

$$
[A, \lambda]=\rho_{S}(A) \lambda, \quad[\lambda, \chi]=\Pi(\lambda, \chi) \in V, \quad A \in \mathfrak{s o}(V), \quad \lambda, \chi \in \mathfrak{g}_{1},
$$

where $\Pi: \mathfrak{g}_{1} \times \mathfrak{g}_{1} \rightarrow V$ is a real, symmetric, $\operatorname{Spin}(t, s)$-equivariant vector-valued bilinear form. ${ }^{30}$

It was shown in [22] that the real, symmetric, $\operatorname{Spin}(t, s)$-equivariant vector-valued bilinear forms on a spinor module form a vector space, which is spanned by vector-valued bilinear forms which can be constructed out of so called super-admissible scalar-valued

\footnotetext{
${ }^{30}$ Note that the required super-Jacobi identity is implied by these conditions.
} 
bilinear forms using Clifford multiplication. Given a bilinear form $\beta$, the associated vectorvalued bilinear form $\Pi_{\beta}$ is defined by

$$
\left\langle\Pi_{\beta}(\lambda, \chi), v\right\rangle=\beta(\gamma(v) \lambda, \chi), \quad \lambda, \chi \in \mathfrak{g}_{1}, v \in V .
$$

Here $\langle\cdot, \cdot\rangle$ is the bilinear form on $V=\mathbb{R}^{t, s}$ and $\gamma(v) \lambda$ is the Clifford multiplication of the vector $v$ with the spinor $\lambda$.

If we denote the generators of $V$ and $\mathfrak{s o}(V)$ by $P_{\mu}$ and $M_{\mu \nu}$, respectively, the relations of the Poincaré Lie algebra are [40]

$$
\begin{aligned}
{\left[P_{\mu}, P_{\nu}\right] } & =0, \quad\left[M_{\mu \nu}, P_{\rho}\right]=i\left(\eta_{\mu \rho} P_{\nu}-\eta_{\nu \rho} P_{\mu}\right), \\
{\left[M_{\mu \nu}, M_{\rho \sigma}\right] } & =i\left(\eta_{\mu \rho} M_{\nu \sigma}-\eta_{\mu \sigma} M_{\nu \rho}-\eta_{\nu \rho} M_{\mu \sigma}+\eta_{\nu \sigma} M_{\mu \rho}\right),
\end{aligned}
$$

where $\eta_{\mu \nu}$ is the Gram matrix of the bilinear form $\langle\cdot, \cdot\rangle$ on $\mathbb{R}^{t, s}$. The generators of the odd part $\mathfrak{g}_{1}$ are the supercharges $Q_{\alpha}$. To translate (A.8) into anti-commutation relations beween supercharges, we expand the spinors and vectors in their respective bases

$$
\lambda=\lambda^{\alpha} Q_{\alpha}, \quad \chi=\chi^{\beta} Q_{\beta}, \quad v=v^{\mu} P_{\mu} .
$$

Clifford multiplication is an operation of $V$ on the spinor module which in terms of components is given by the action of $\gamma$-matrices on spinors,

$$
P^{\mu} Q_{\alpha}=\gamma_{\alpha}^{\mu}{ }_{\alpha}^{\beta} Q_{\beta}
$$

Therefore

$$
\gamma: V \times \mathfrak{g}_{1} \rightarrow \mathfrak{g}_{1}, \quad(v, \lambda) \mapsto \gamma_{v} \lambda=v_{\mu} P^{\mu} \lambda=v_{\mu} \gamma^{\mu \alpha}{ }_{\beta} \lambda^{\beta} Q_{\alpha},
$$

where $\left(\gamma_{\beta}^{\mu \alpha}\right)=\left(\gamma^{\mu}\right)^{T}=\tau C \gamma^{\mu} C^{-1}$ is the transposed of $\gamma^{\mu}{ }^{31}$ Therefore

$$
\beta(\gamma(v) \lambda, \chi)=v_{\mu} \gamma_{\gamma}^{\mu \alpha} \lambda^{\gamma} \chi^{\beta} \beta\left(Q_{\alpha}, Q_{\beta}\right) .
$$

A non-degenerate bilinear form can be used to identify a module with its dual (also called transposed module). Since $\beta$ is required to be an admissible bilinear form this isomorphism is spin-equivariant, and induces a map which maps the $\gamma$-matrices to their transposed [12]. In other words the Gram matrix $\beta\left(Q_{\alpha}, Q_{\beta}\right)$ of the bilinear form $\beta$ can be interpreted as a charge conjugation matrix $C$. With our index conventions the Gram matrix $\beta\left(Q_{\alpha}, Q_{\beta}\right)$ is the inverse charge conjugation matrix $C^{-1}=\left(C_{\alpha \beta}\right)$. Comparing to (A.8) we conclude that

$$
\Pi_{\beta}(\lambda, \chi)=\left[\lambda^{\beta} \chi^{\gamma}\left(\gamma^{\mu}\right)_{\gamma}^{\alpha} C_{\alpha \beta}\right] P_{\mu}=\sigma_{C}\left[\lambda^{\beta} \chi^{\gamma} C_{\beta \alpha}\left(\gamma^{\mu}\right)_{\gamma}^{\alpha}\right] P_{\mu}=\sigma_{C} \tau_{C}\left[\lambda^{\beta} \chi^{\gamma}\left(\gamma^{\mu}\right)_{\beta}{ }^{\alpha} C_{\alpha \gamma}\right] P_{\mu}
$$

Here we used $C^{T}=\sigma C$ and $\left(\gamma^{\mu}\right)^{T}=\tau C \gamma^{\mu} C^{-1}$. Defining the superbracket using the vector-valued bilinear form, $\{\lambda, \chi\}=\Pi_{\beta}(\lambda, \chi)$, we obtain

$$
\left\{Q_{\beta}, Q_{\gamma}\right\}=\sigma \tau\left(\gamma^{\mu} C^{-1}\right)_{\beta \gamma} P_{\mu} .
$$

\footnotetext{
${ }^{31}$ Note that for $\tau=-1$ the matrices $\gamma^{\mu}$ and $\left(\gamma^{\mu}\right)^{T}$ are not related by raising/lowering indices using $C$ (extra sign).
} 
For this to be a symmetric bracket we need that

$$
\left(\gamma^{\mu} C^{-1}\right)^{T}=C^{-1, T}\left(\gamma^{\mu}\right)^{T}=\sigma C^{-1} \tau C \gamma^{\mu} C^{-1}=\sigma \tau \gamma^{\mu} C^{-1}=\gamma^{\mu} C^{-1},
$$

that is $\sigma \tau=1$. This shows explicitly how the symmetry of the superbracket is related to the super-admissibility of the bilinear form $\beta$.

While we are ultimately interested in real supersymmetry algebras, all the above concepts and statement naturally extend to complex Poincaré Lie superalgebras. Of course, for complex bilinear forms the concept of signature loses its invariant meaning. We can use this to pass from a real algebra to its complexification, and from there to other real forms.

In general, the spinorial module $\mathfrak{g}_{1}$ can be reducible. For sums of inequivalent modules the contributions to the supersymmetry anti-commutator just add up 'incoherently', while for sums of equivalent modules they can 'mix.' This is what gives rise to the larger Rsymmetry groups of extended supersymmetry algebras. Let us consider the case of $\mathcal{N}$ copies of an irreducible module $S$, where $\mathfrak{g}_{1}=S \oplus \cdots \oplus S=\mathcal{N} S=S \otimes_{\mathbb{K}} \mathbb{K}^{\mathcal{N}}$, where $\mathbb{K}$ is $\mathbb{R}$ or $\mathbb{C}$, depending on whether $S$ is a real or complex module. Denoting irreducible spinor indices by $\alpha, \beta, \ldots$ and labeling copies by $i, j=1, \ldots \mathcal{N}$, the supercharges are $Q_{i \alpha}$ and spinors expand as $\lambda=\lambda^{i \alpha} Q_{i \alpha}$. The bilinear form on $S \otimes_{\mathbb{K}} \mathbb{K}^{\mathcal{N}}$ is $\beta=C \otimes M$, where $C$ is the bilinear form defined by the charge conjugation matrix on the irreducible module $S$, and where $M$ is a symmetric or antisymmetric bilinear form on the multiplicity space $\mathbb{K}^{\mathcal{N}}$. By a similar computation to the one above we find

$$
\beta\left(\gamma^{\mu} \lambda, \chi\right)=\sigma \tau \lambda^{i \alpha} \chi^{j \beta} \gamma_{\alpha}^{\mu}{ }^{\gamma} C_{\gamma \beta} M_{j i}=\sigma \tau \sigma_{M} \lambda^{i \alpha} \chi^{j \beta}\left(\gamma^{\mu} C^{-1}\right)_{\alpha \beta} M_{i j}
$$

where $\sigma_{M}$ is the symmetry of $M$, that is $M^{T}=\sigma_{M} M$. Therefore

$$
\left\{Q_{i \alpha}, Q_{j \beta}\right\}=\sigma \tau \sigma_{M}\left(\gamma^{\mu} C^{-1}\right)_{\alpha \beta} M_{i j} P_{\mu} .
$$

The bracket is symmetric if

$$
\left(\gamma^{\mu} C^{-1}\right)_{\alpha \beta} M_{i j}=\left(\gamma^{\mu} C^{-1}\right)_{\beta \alpha} M_{j i},
$$

which requires $\sigma \tau \sigma_{M}=1$. Since the $\gamma$-matrices do not act on $\mathbb{K}^{\mathcal{N}}$, it follows that $\sigma \tau \sigma_{M}=$ $\sigma_{\beta} \tau_{\beta}$, that is the bracket is symmetric if the bilinear form $\beta=C \otimes M$ is super-admissible.

To summarize, given a super-admissible bilinear form $\beta=C \otimes M$,

$$
\beta(\lambda, \chi)=\lambda^{i \alpha} C_{\alpha \beta} \chi^{j \beta} M_{j i}
$$

the corresponding anti-commutation relations are

$$
\left\{Q_{i \alpha}, Q_{j \beta}\right\}=\left(\gamma^{\mu} C^{-1}\right)_{\alpha \beta} M_{i j} P_{\mu} .
$$

For a given charge conjugation matrix $C$ one has either $\sigma \tau=1$ or $\sigma \tau=-1$. Therefore a charge conjugation matrix does not always define a supersymmetry algebra. However, by taking an even number of copies and pairing $C$ with an antisymmetric bilinear form on the multiplicity space we can obtain a super-admissible bilinear form on the extended spinor module. Our approach is to always double the spinor module and to use a super-admissible 
bilinear form on the doubled space to define a complex algebra with relations (A.21). This uses that the doubling can be interpreted as a complexification. Real algebras are obtained by choosing a real form through imposing a reality condition of the form

$$
\left(\lambda^{i}\right)^{*}=\alpha B \lambda^{j} L_{j i}
$$

where $\alpha$ is a phase and where $B \otimes L$ defines a real structure $\rho$ on $\mathfrak{g}_{1} \oplus \mathfrak{g}_{1} \cong \mathfrak{g}_{1} \otimes_{\mathbb{R}} \mathbb{C} \cong \mathfrak{g}_{1}^{\mathbb{C}}$.

\section{A.4 Useful formulae relating to chirality}

The following formula are used extensively throughout this paper and are provided here for easy reference. They can be found in, or straightforwardly obtained from [17, 25].

$$
\begin{aligned}
C_{ \pm} \gamma_{*} & = \begin{cases} \pm i C_{\mp}, & D=2,6,10, \\
C_{\mp}, & D=4,8,12,\end{cases} \\
\gamma_{*} C_{ \pm} & = \begin{cases} \pm i C_{\mp}, & D=2,6,10, \\
-C_{\mp}, & D=4,8,12,\end{cases} \\
B_{ \pm} \gamma_{*} & = \begin{cases} \pm i \sigma_{+} \sigma_{-} B_{\mp}=\mp i B_{\mp}, & D=2,6,10, \\
\sigma_{+} \sigma_{-} B_{\mp}=B_{\mp}, & D=4,8,12,\end{cases} \\
\gamma_{*} B_{ \pm} & = \begin{cases}(-1)^{t} \mp i \sigma_{+} \sigma_{-} B_{\mp}= \pm(-1)^{t} i B_{\mp}, & D=2,6,10, \\
(-1)^{t} \sigma_{+} \sigma_{-} B_{\mp}=(-1)^{t} B_{\mp}, & D=4,8,12,\end{cases} \\
B_{ \pm}^{*} \gamma_{*} & = \begin{cases} \pm i B_{\mp}^{*}, & D=2,6,10, \\
B_{\mp}^{*}, & D=4,8,12 .\end{cases}
\end{aligned}
$$

For completeness we note that, as shown in the main text, the symmetry $\sigma_{ \pm}$of $C_{ \pm}$satisfies $\sigma_{+}=-\sigma_{-}$in the Weyl compatible dimensions $D=2,6,10$, and $\sigma_{+}=\sigma_{-}$in the Weyl incompatible dimensions $D=4,8,12$.

\section{A.5 Proof that a signature flip $(t, s) \leftrightarrow(s, t)$ exchanges $B_{+} \leftrightarrow B_{-}$}

This is the proof of a statement that we used in the main part of this paper. Consider the $(t, s)$ signature $\gamma$-matrices, which obey

$$
\left(\gamma_{i}\right)^{2}= \begin{cases}-1, & i \leq t, \\ +1, & i>t .\end{cases}
$$

We define the $(s, t)$ signature $\gamma$-matrices as $\gamma_{m}^{\prime}=i \gamma_{(D-m+1)}($ where $D=t+s)$ such that they correctly obey

$$
\left(\gamma_{i}^{\prime}\right)^{2}= \begin{cases}-1, & i \leq s \\ +1, & i>s .\end{cases}
$$

Both theories have the same charge conjugation matrices, $C_{+}$and $C_{-}$and $A$-matrices

$$
\begin{aligned}
& A^{(t, s)}=\gamma_{1} \ldots \gamma_{t}, \\
& A^{(s, t)}=\gamma_{1}^{\prime} \ldots \gamma_{s}^{\prime}=i^{s} \gamma_{D} \ldots \gamma_{t+1} .
\end{aligned}
$$


We then see that, using $C_{+}=k C_{-} \gamma_{*}(k$ is the constant from (A.23))

$$
\begin{aligned}
B_{+}^{(t, s)} & =\left(C_{+}\left(A^{(t, s)}\right)^{-1}\right)^{T} \\
& =\left(k C_{-} \gamma_{*}\left(A^{(t, s)}\right)^{-1}\right)^{T} .
\end{aligned}
$$

Using our definitions for $A^{(t, s)}$ we find

$$
\begin{aligned}
\gamma_{*}\left(A^{(t, s)}\right)^{-1} & =(-i)^{t} \gamma_{1} \ldots \gamma_{D}(-1)^{t} \gamma_{t} \ldots \gamma_{1} \\
& =(-1)^{s t}(-i)^{t} \gamma_{t+1} \ldots \gamma_{D} \\
& =(-1)^{s t}(-i)^{t}(-i)^{s} \gamma_{s}^{\prime} \ldots \gamma_{1}^{\prime} \\
& =(-1)^{s t}(-i)^{D}\left(A^{(s, t)}\right)^{-1}=(-1)^{s t+\frac{D}{2}}\left(A^{(s, t)}\right)^{-1}
\end{aligned}
$$

such that

$$
\begin{aligned}
B_{+}^{(t, s)} & =\left(k C_{-}(-1)^{s t+\frac{D}{2}}\left(A^{(s, t)}\right)^{-1}\right)=k(-1)^{s t+\frac{D}{2}} B_{-}^{(s, t)} \\
\Longrightarrow \quad\left(B_{+}^{(t, s)}\right)^{*} B_{+}^{(t, s)} & =\left(B_{-}^{(s, t)}\right)^{*} B_{-}^{(s, t)} .
\end{aligned}
$$

\section{B Real (semi-)spinors and Majorana spinors}

In this appendix we summarize the relation between complex and real spinors and semispinors as defined in the mathematics literature and Dirac, Weyl, Majorana and MajoranaWeyl spinors as defined in the physics literature. The complex and real spinor module $\mathbb{S}$ and $S$ are defined by restricting irreducible representations of the complex and real Clifford algebras $\mathbb{C l}_{D}$ and $C l_{t, s}$ to the real spin group $\operatorname{Spin}(t, s)$. As $\operatorname{Spin}(t, s)$-modules $\mathbb{S}$ and $S$ can be isomorphic or non-isomorphic. Complex spinors $\psi \in \mathbb{S}$ are Dirac spinors, while real spinors $\psi \in S$ are not always Majorana spinors. The following cases can occur, depending on dimension and signature.

1. $\mathbb{S} \not S$, the complex and real spinor module are not isomorphic. Then $\mathbb{S}$ is the complexification of $S, \mathbb{S}=S \otimes_{\mathbb{R}} \mathbb{C}$ and $S$ is a real subset of $\mathbb{S}$ fixed under the action of an invariant real structure $\rho, S=\mathbb{S}^{\rho}$. The elements of $S$ are Majorana spinors. $S$ can be irreducible or reducible.

(a) $S$ is irreducible. Then Majorana spinors are the unique irreducible spinor representation. If, in addition, the dimension is even, Majorana spinors are equivalent (as real $\operatorname{Spin}(t, s)$ representations) to Weyl spinors: $S \cong \mathbb{S}_{+} \cong \mathbb{S}_{-}$. This is, for example, the case in signature $(1,3)$.

(b) $S$ is reducible. Then real spinors decompose into real semi-spinors, $S=S_{+}+S_{-}$. Given that $\mathbb{S}=S \otimes_{\mathbb{R}} \mathbb{C}$, the real semi-spinor modules must be non-isomorphic, since otherwise their complexifications $S_{ \pm} \otimes_{\mathbb{R}} \mathbb{C}$ would be isomorphic as complex modules, which is not true. The real semi-spinors $S_{+} \nsucceq S_{-}$are Majorana-Weyl spinors. As is well known, Majorana-Weyl spinors exist if and only if $t-s$ is 0 modulo 8. In table 1 these are the entries where $\left(\epsilon_{+}, \epsilon_{-}\right)=(1,1)$. 
2. $\mathbb{S} \cong S$, the complex and real spinor module are isomorphic. Here we have three subcases:

(a) $S$ is irreducible. Then Majorana spinors don't exist ( $\mathbb{S}$ does not admit an invariant real structure) and Dirac spinors are the unique irreducible spinor representation. This is realized in signature $(1,4)$.

(b) $S=S_{+}+S_{-}, S_{+} ¥ S_{-} . S$ is reducible and decomposes into non-isomorphic semi-spinor modules. In this case real semi-spinors are the same as complex semi-spinors, that is Weyl spinors, $S_{ \pm} \cong \mathbb{S}_{ \pm}$. This happens in even dimensions in those signatures where no invariant real structures, and hence no Majorana spinors exist, for example in signature $(4,0)$ or $(0,4)$.

(c) $S=S_{+}+S_{-}, S_{+} \cong S_{-} . S$ is reducible, and decomposes into isomorphic semispinor modules. In this case $\mathbb{S} \cong S \cong S_{ \pm} \otimes \mathbb{C}$ carries an invariant real structure. The elements of $S_{ \pm}$are Majorana spinors, and we are in a signature where no Majorana-Weyl spinors exist. There are two subcases:

i. If the dimension is even, then complex and real semi-spinors coincide and all types semi-spinor modules are isomorphic as real modules: $\mathbb{S}_{+} \cong \mathbb{S}_{-} \cong$ $S_{+} \cong S_{-}$. This happens, for example, in signature $(3,1)$.

ii. If the dimension is odd, no Weyl spinors exist, and we have $\mathbb{S}=S=S_{ \pm} \otimes_{\mathbb{R}} \mathbb{C}$. This is realized, for example, in signature $(3,2)$.

More details for the explicit examples we have mentioned can be found in $[16,17]$.

\section{Details on the complexification of spinor modules}

In this appendix we provide details on the complexification of the odd parts $\mathfrak{g}_{1}=S^{\oplus N} \cong$ $S \otimes \mathbb{R}^{N}, \mathfrak{g}_{1}=S_{+}^{\oplus N} \cong S_{+} \otimes \mathbb{R}^{N}$ and $\mathfrak{g}_{1}=S_{+}^{\oplus N_{+}} \oplus S_{-}^{\oplus N_{-}} \cong S_{+} \otimes \mathbb{R}^{N_{+}} \oplus S_{-} \otimes \mathbb{R}^{N_{-}}$of real supersymmetry algebras.

We start with the simpler case where $D$ is odd and no Weyl spinors exist. The unique real irreducible spinor representation is either $S$ or $S_{+} \cong S_{-}$. There are two cases.

1. $S$ is irreducible. Then

$$
\mathfrak{g}_{1} \otimes_{\mathbb{R}} \mathbb{C}=\left(S \otimes_{\mathbb{R}} \mathbb{C}\right)^{\oplus N} \cong\left(S \otimes_{\mathbb{R}} \mathbb{C}\right) \otimes_{\mathbb{C}} \mathbb{C}^{N} .
$$

There are two subcases.

(a) $S \cong \mathbb{S}$. The smallest spinor representation is given by Dirac spinors, and

$$
\mathfrak{g}_{1} \otimes_{\mathbb{R}} \mathbb{C}=\left(\mathbb{S} \otimes_{\mathbb{R}} \mathbb{C}\right)^{\oplus N} \cong\left(\mathbb{S} \otimes_{\mathbb{R}} \mathbb{C}\right) \otimes_{\mathbb{C}} \mathbb{C}^{N} \cong \mathbb{S} \otimes_{\mathbb{C}} \mathbb{C}^{2 N}
$$

This case is characterized by $\mathbb{S}$ not admitting an invariant real structure.

(b) $S \otimes_{\mathbb{R}} \mathbb{C} \cong \mathbb{S}$. The smallest spinor representation is given by Majorana spinors, and

$$
\mathfrak{g}_{1} \otimes_{\mathbb{R}} \mathbb{C}=\left(S \otimes_{\mathbb{R}} \mathbb{C}\right)^{\oplus N} \cong \mathbb{S} \otimes_{\mathbb{C}} \mathbb{C}^{N}
$$

This case is characterized by $\mathbb{S}$ admitting an invariant real structure. 
2. $S$ is reducible and $S_{+} \cong S_{-}$. Then $\mathbb{S} \cong S \cong S_{ \pm} \otimes_{\mathbb{R}} \mathbb{C}$. The unique irreducible spinor representation given by Majorana spinors $S_{+} \cong S_{-}$, and

$$
\mathfrak{g}_{1} \otimes_{\mathbb{R}} \mathbb{C}=\left(S_{+} \otimes_{\mathbb{R}} \mathbb{C}\right)^{\oplus N} \cong \mathbb{S} \otimes_{\mathbb{C}} \mathbb{C}^{N}
$$

In this case $\mathbb{S}$ admits an invariant real structure.

If $D$ is even, then there is one additional case to consider, namely when $S$ is reducible and the real semi-spinor modules are non-isomorphic, $S_{+} \cong S_{-}$. In this case the minimal spinors are either Weyl spinors or Majorana-Weyl spinors. Since there are two inequivalent irreducible real spinor representations, the general form of the odd part of the supersymmetry algebra is $\mathfrak{g}_{1}=S_{+}^{\oplus N_{+}} \oplus S_{-}^{\oplus N_{-}}$. The additional third case is

3. $S=S_{+}+S_{-}, S_{+} \cong S_{-}$. There are two sub-cases:

1. $\mathbb{S}_{ \pm} \cong S_{ \pm}$. In this case $\mathbb{S}_{ \pm}$do not admit invariant real structures and the inequivalent minimal spinors are Weyl spinors.

2. $\mathbb{S}_{ \pm} \cong S_{ \pm} \otimes_{\mathbb{R}} \mathbb{C}$. In this case $\mathbb{S}_{ \pm}$admit invariant real strucutures, and the inequivalent minimal spinors are Majorana-Weyl spinors.

In both cases the complexification procedes analogous to case 1 , with $\mathbb{S}$ replaced by $\mathbb{S}_{+}$and $\mathbb{S}_{-}$.

\section{Matrix notation for Weyl spinors}

\section{D.1 Explanation of the matrix notation}

In this section, we will describe a notation which was introduced in [17], and makes calculations easier when dealing with Weyl spinors in even dimensions. Using the natural embedding $\mathbb{S}_{ \pm} \subset \mathbb{S}$ we combine the two Weyl spinor modules into a single 'doubled-again' spinor module

$$
\left(\lambda^{I}\right)=\left(\lambda_{+}^{i}, \lambda_{-}^{\hat{i}}\right)=\left(\lambda_{+}^{1}, \ldots, \lambda_{+}^{K_{+}}, \lambda_{-}^{1}, \ldots, \lambda_{-}^{K_{-}}\right) \in \mathbb{S}_{+}^{\oplus K_{+}} \oplus \mathbb{S}_{-}^{\oplus K_{-}} \subset \mathbb{S}^{\oplus\left(K_{+}+K_{-}\right)}=\mathbb{S} \otimes \mathbb{C}^{K_{+}+K_{-}}
$$

Using a mixture of matrix notation and index notation, orthogonal and isotropic bilinear forms take the form

$$
\begin{array}{ll}
\left(\bar{\lambda}_{+}^{i}, \bar{\lambda}_{-}^{\hat{i}}\right)\left(\begin{array}{cc}
M_{j i} & 0 \\
0 & M_{\hat{j} \hat{i}}^{\prime}
\end{array}\right)\left(\begin{array}{c}
\chi_{+}^{j} \\
\chi_{-}^{\hat{j}}
\end{array}\right) & \text { (Orthogonal) } \\
\left(\bar{\lambda}_{+}^{i}, \bar{\lambda}_{-}^{i}\right)\left(\begin{array}{cc}
0 & M_{j i} \\
M_{j i} & 0
\end{array}\right)\left(\begin{array}{c}
\chi_{+}^{j} \\
\chi_{-}^{j}
\end{array}\right) & \text { (Isotropic). }
\end{array}
$$

In the orthogonal case $M$ is the bilinear form on the $\mathbb{C}^{K_{+}}$factor, $M^{\prime}$ the one on the $\mathbb{C}^{K_{-}}$ factor, $i, j=1, \ldots, K_{+}$and $\hat{i}, \hat{j}=1, \ldots, K_{-}$. For isotropic signatures, necessarily $M=M^{\prime}$ and $K_{+}=K_{-}$. 
In addition, we have a real structure, $\rho$, which is either Weyl-compatible or Weylincompatible. For a Weyl-compatible reality condition, we write this as

$$
\begin{aligned}
\rho\left(\lambda_{+}^{i}\right) & =\alpha^{*} B^{*}\left(\lambda_{+}^{j}\right)^{*} L_{j i}, \quad \rho\left(\lambda_{-}^{\hat{i}}\right)=\beta^{*} B^{*}\left(\lambda_{-}^{\hat{j}}\right)^{*} L_{\hat{j} \hat{i}}^{\prime} \\
\rightarrow \quad \rho\left(\begin{array}{c}
\lambda_{+}^{i} \\
\lambda_{-}^{\hat{i}}
\end{array}\right) & =\left(\begin{array}{cc}
\alpha^{*} B^{*} L_{j i} & 0 \\
0 & \beta^{*} B^{\prime *} L_{\hat{j} \hat{i}}^{\prime}
\end{array}\right)\left(\begin{array}{l}
\lambda_{+}^{j} \\
\lambda_{-}^{j}
\end{array}\right)^{*} .
\end{aligned}
$$

Here $B$ and $B^{\prime}$ can refer to either $B_{ \pm}$.

Weyl-incompatible reality conditions are written as

$$
\rho\left(\lambda_{ \pm}^{i}\right)=\alpha^{*} B^{*}\left(\lambda_{\mp}^{j}\right)^{*} L_{j i} \rightarrow \rho\left(\begin{array}{c}
\lambda_{+}^{i} \\
\lambda_{-}^{i}
\end{array}\right)=\alpha^{*} B^{*}\left(\begin{array}{cc}
0 & L_{j i} \\
L_{j i} & 0
\end{array}\right)\left(\begin{array}{c}
\lambda_{+}^{j} \\
\lambda_{-}^{j}
\end{array}\right)^{*} .
$$

Often it is possible and convenient to suppress the indices $i, j$, and write expressions in terms of vectors-of-vectors and block matrices

$$
\begin{aligned}
& \left(\underline{\bar{\lambda}}_{+}, \underline{\bar{\lambda}}_{-}\right)\left(\begin{array}{cc}
M & 0 \\
0 & M^{\prime}
\end{array}\right)\left(\underline{\underline{\chi}}_{+}\right) \quad \text { (Orthogonal) } \\
& \left(\underline{\bar{\lambda}}_{+}, \underline{\bar{\lambda}}_{-}\right)\left(\begin{array}{cc}
0 & M \\
M & 0
\end{array}\right)\left(\underline{\chi}_{+} \underline{\chi}_{-}\right) \quad \text { (Isotropic). }
\end{aligned}
$$

When we do this, we will change the indices such that normal matrix multiplication makes sense for the resulting expressions. This induces a sign if $M=J$ is the anti-symmetric bilinear form with components $J_{i j}=-J_{j i}$ :

$$
\left(\bar{\lambda}_{+}^{i}, \bar{\lambda}_{-}^{i}\right)\left(\begin{array}{cc}
0 & J_{j i} \\
J_{j i} & 0
\end{array}\right)\left(\begin{array}{c}
\chi_{+}^{j} \\
\chi_{-}^{j}
\end{array}\right)=\left(\bar{\lambda}_{+}, \bar{\lambda}_{-}\right)\left(\begin{array}{cc}
0 & -J \\
-J & 0
\end{array}\right)\left(\begin{array}{l}
\underline{\chi}_{+} \\
\underline{\chi}_{-}
\end{array}\right) .
$$

For a final example, (D.5) is rewritten as

$$
\rho\left(\begin{array}{l}
\underline{\lambda}_{+} \\
\underline{\lambda}_{-}
\end{array}\right)=B^{*}\left(\begin{array}{cc}
\alpha^{*} L & 0 \\
0 & \beta^{*} L^{\prime}
\end{array}\right)\left(\begin{array}{l}
\underline{\lambda}_{+} \\
\underline{\lambda}_{-}
\end{array}\right) .
$$

When using this notation the Schur group and consequently the R-symmetry group acts by linear transformations on the expanded internal space $\mathbb{C}^{K_{+}+K_{-}}$, but not on the spinor indices. This disentangling of spinor and internal indices with respect to the action of the Schur group is the main advantage of this notation. Without the additional doubling, the Schur group can act non-trivially on spinors indices in even dimensions by acting differently on spinors depending on their chirality. For example, in signature $(1,3)$ positive and negative chirality spinors carry opposite charge under $\mathrm{U}(1) \subset G_{R}=\mathrm{U}(2)$, see [12]. By doubling the auxiliary space, any chiral action of the Schur group is encoded in the larger matrix acting on the doubled space. Afterwards, the effects on each Weyl spinor module can be reconstructed and rewritten in terms of Id and $\gamma_{*}$ acting on the original spinor module. 


\section{D.2 Action of R-symmetry transformations on the complex spinor module $\mathbb{S}$}

In even dimensions R-symmetry transformations act non-trivially on the complex spinor module $\mathbb{S}$, though only through a relative sign between complex semi-spinors, since Rsymmetry transformations by definition commute with the Lie algebra of the spin group. We have shown that the actions of the spin group and R-symmetry group can still be disentangled by doubling the internal space. In the following section we briefly describe how our results translate to a more conventional notation, where we employ Dirac spinors and do not double the internal space. R-symmetry transformations are then given by products of actions on the internal space with an action of $\mathbb{1}, \gamma_{*}$ on Dirac spinors.

The chirality matrix $\gamma_{*}$ acts on the Weyl spinors $\lambda_{ \pm} \in \mathbb{S}_{ \pm}$as $\gamma_{*} \lambda_{ \pm}= \pm \lambda_{ \pm}$. We choose a basis of $\mathbb{S}$ where

$$
\gamma_{*}=\left(\begin{array}{cc}
\mathbb{1} & 0 \\
0 & -\mathbb{1}
\end{array}\right)
$$

where $\mathbb{1}$ is the identity matrix acting on complex semi-spinors $\mathbb{S}_{ \pm}$. In matrix notation, $\gamma_{*} \lambda_{ \pm}^{i}= \pm \lambda_{ \pm}^{i}$ acts on $\left(\lambda_{+}^{i}, \lambda_{-}^{i}\right)$ as the matrix

$$
\left(\begin{array}{cc}
\mathbb{1}_{K} & 0 \\
0 & -\mathbb{1}_{K}
\end{array}\right)=\gamma_{*} \otimes \mathbb{1}_{K}
$$

\section{D.2.1 Orthogonal bilinear forms}

In orthogonal Weyl-compatible signatures the R-symmetry transformations act independently on each Weyl spinor module and are specified by the action on the internal $\mathbb{C}^{K_{ \pm}}$ factor. This is because the Weyl spinor modules are complex irreducible modules (so we can apply Schur's lemma exactly like in odd dimensions) and the reality condition is defined on a Weyl spinor module alone. If $K_{+} \neq K_{-}$we cannot combine Weyl spinors into Dirac spinors.

When $K_{+}=K_{-}$, we can combine the Weyl spinors into Dirac spinors. Then R-symmetry transformations act as follows. We know that a general element of the R-symmetry Lie algebra has the form

$$
r=\left(\begin{array}{ll}
a & 0 \\
0 & b
\end{array}\right),
$$

where $a, b$ are Lie algebra elements for the factor of the R-symmetry which acts on spinors of given chirality. We can rewrite this as

$$
r=\left(\begin{array}{ll}
c & 0 \\
0 & c
\end{array}\right)+\left(\begin{array}{cc}
d & 0 \\
0 & -d
\end{array}\right)=(\operatorname{Id} \otimes c)+\left(\gamma_{*} \otimes d\right),
$$

for $c=\frac{1}{2}(a+b)$ and $d=\frac{1}{2}(a-b)$.

We can see that, at most, R-symmetry generators act as identity or $\gamma_{*}$ on the $\mathbb{S}$ factor. In this case, the re-writing is somewhat artificial since the Lie algebra elements $a, b$ are independent. But in the remaining cases, where we will do something similar, the two transformations will depend on one another. 
For a Weyl-incompatible orthogonal signature, the reality condition links the two chiralities, and we found that they take the form

$$
r=\left(\begin{array}{cc}
a & 0 \\
0 & L a^{*} L^{-1}
\end{array}\right),
$$

where $a$ acts entirely on $\mathbb{S}_{+}$while the corresponding transformation on $\mathbb{S}_{-}$is $L a^{*} L^{-1}$. This can be recast into transformations that act on the entire spinor module $\mathbb{S}=\mathbb{S}_{+}+\mathbb{S}_{-}$. Using that conjugation by $L$ and complex conjugation are involutions, we can rewrite $a$ and $L a^{*} L^{-1}$ in terms of

$$
a_{ \pm}=\frac{1}{2}\left(a \pm L a^{*} L^{-1}\right),
$$

so that

$$
r=\left(\begin{array}{cc}
a_{+} & 0 \\
0 & a_{+}
\end{array}\right)+\left(\begin{array}{cc}
a_{-} & 0 \\
0 & -a_{-}
\end{array}\right)=\left(\mathbb{1} \otimes a_{+}\right)+\left(\gamma_{*} \otimes a_{-}\right) .
$$

This is slightly different from the previous case with Weyl-compatible signatures because $a_{+}$and $a_{-}$are functions of $a$ alone. However we see that similarly the generators of the R-symmetry group can be written in a way where they act either as Id or $\gamma_{*}$ on $\mathbb{S}$.

\section{D.2.2 Isotropic bilinear forms}

For an isotropic vector-valued bilinear form, $\gamma_{*}$ generates a real one-parameter subgroup of the R-symmetry group. Consider the transformation

$$
\lambda^{i} \rightarrow e^{\omega \gamma_{*}} \lambda^{i}=e^{\omega} \lambda_{+}^{i}+e^{-\omega} \lambda_{-}^{i},
$$

where $\omega \in \mathbb{C}$. A complex vector-valued bilinear form is invariant under this transformation:

$$
\begin{aligned}
\beta\left(\gamma^{\mu} \lambda, \chi\right) & =\left(\gamma^{\mu} \lambda_{+}^{i}\right)^{T} C \chi_{-}^{j} M_{j i}+\left(\gamma^{\mu} \lambda_{-}^{i}\right)^{T} C \chi_{+}^{j} M_{j i} \\
& \rightarrow\left(\gamma^{\mu} e^{\omega} \lambda_{+}^{i}\right)^{T} C e^{-\omega} \chi_{-}^{j} M_{j i}+\left(\gamma^{\mu} e^{\omega} \lambda_{-}^{i}\right)^{T} C e^{-\omega} \chi_{+}^{j} M_{j i}=\beta\left(\gamma^{\mu} \lambda, \chi\right) .
\end{aligned}
$$

In matrix notation the transformation in (D.18) is

$$
\left(\begin{array}{l}
\underline{\lambda}_{+} \\
\underline{\lambda}_{-}
\end{array}\right) \rightarrow \exp \left(\omega \gamma_{*} \otimes \mathbb{1}_{K}\right)\left(\begin{array}{l}
\underline{\lambda}_{+} \\
\underline{\lambda}_{-}
\end{array}\right) .
$$

Imposing that (D.18) commutes with the reality condition in isotropic dimensions forces $\omega$ to be real in Weyl-compatible signatures and to be imaginary in Weyl-incompatible signatures. The corresponding one-dimensional subgroups $\mathrm{SO}(1,1)$ and $\mathrm{U}(1)$ of the $\mathrm{R}$ symmetry group are often generated when performing a dimensional reduction from odd to even dimensions. For example, the reduction of a supersymmetry algebra based on a single Dirac spinor from five to four dimensions increases the R-symmetry group from $\mathrm{SU}(2)$ to $\mathrm{U}(2) \cong_{\text {local }} \mathrm{SU}(2) \times \mathrm{U}(1)$ for the reduction $(t, s)=(1,4) \rightarrow(1,3)$ and to $\mathrm{U}^{*}(2) \cong_{\text {local }}$ $\mathrm{SU}(2) \times \mathrm{SO}(1,1)$ for the reduction $(t, s)=(1,4) \rightarrow(0,4)$ [12]. General R-symmetry elements 
act simultaneously as Id or $\gamma_{*}$ on $\mathbb{S}$ and by a non-trivial transformation on the $\mathbb{C}^{K}$ factor, therefore the R-symmetry groups are only locally isomorphic to direct products.

The general form of an R-symmetry transformation in isotropic signatures is

$$
r=\left(\begin{array}{cc}
a & 0 \\
0 & -M^{-1} a^{T} M
\end{array}\right) .
$$

Since conjugation by $M$ and transposition are both involutions, that we can split $a$ into eigen-matrices under the combination of these two operations

$$
a=a_{+}+a_{-}, \quad \text { with } \quad a_{ \pm}=\frac{1}{2}\left(a \pm M^{-1} a^{T} M\right) .
$$

Therefore we can write

$$
r=\left(\begin{array}{cc}
a_{+} & 0 \\
0 & -a_{+}
\end{array}\right)+\left(\begin{array}{cc}
a_{-} & 0 \\
0 & a_{-}
\end{array}\right)=\left(\gamma_{*} \otimes a_{+}\right)+\left(\mathbb{1} \otimes a_{-}\right) .
$$

From this, we conclude that in isotropic signatures the only possible non-trivial action of the R-symmetry generators on $\mathbb{S}$ is through multiplication by $\gamma_{*}$.

\section{E Details of isomorphisms}

In this appendix we provide the details of the proof that real supersymmetry algebras constructed using the data $(C, M, B, L)$ on $\mathfrak{g}_{1}^{\mathbb{C}}$ are classified by their R-symmetry group (together with a choice of the relative sign between $\alpha_{+}$and $\alpha_{-}$for orthogonal Weylcompatible signatures). This involves showing that certain sets of data can be swapped without changing the complex superbracket and the reality condition. For this purpose it is useful to introduce certain transformations, denoted $R, S_{L}, T$ which allow one to establish the required isomorphisms for orthogonal and isotropic Weyl-compatible signatures. The isotropic Weyl incompatible case is a bit more involved and is therefore treated separately.

\section{E.1 The R-transformation}

We define an invertible map on $\mathfrak{g}_{1}^{\mathbb{C}}$ by

$$
R: \quad \lambda^{i} \mapsto \Psi^{i}=\frac{1}{\sqrt{2}}\left(1+i \gamma_{*}\right) \lambda^{i} .
$$

For reference, the inverse is given by

$$
\lambda^{i}=\frac{1}{\sqrt{2}}\left(1-i \gamma_{*}\right) \Psi^{i}
$$

\section{E.1.1 Orthogonal dimensions}

We claim that in orthogonal dimensions the R-transformation exchanges the bilinear forms $C_{+} \otimes M$ and $C_{-} \otimes M$ and preserves the reality condition up to an overall phase factor.

To show this consider the chiral projections of the spinors,

$$
\Psi_{ \pm}^{i}=\frac{1}{\sqrt{2}}\left(1+i \gamma_{*}\right) \lambda_{ \pm}^{i}=\frac{1}{\sqrt{2}}(1 \pm i) \lambda_{ \pm}^{i} .
$$


Recall that for an orthogonal bilinear form, if $C_{+} \otimes M$ is super-admissible then so is $C_{-} \otimes M$. Using (A.23) and (A.25) we find these two super-admissible bilinear forms are related by

$$
\left(C_{+} \otimes M\right)\left(\gamma^{\mu} \underline{\lambda}_{ \pm}, \underline{\chi}_{ \pm}\right)= \pm i\left(C_{-} \otimes M\right)\left(\gamma^{\mu} \underline{\lambda}_{ \pm}, \underline{\chi}_{ \pm}\right) .
$$

The R-transformation removes this factor of $\pm i$ :

$$
\left(C_{+} \otimes M\right)\left(\gamma^{\mu} \underline{\lambda}_{ \pm}, \underline{\chi}_{ \pm}\right)=\left(C_{-} \otimes M\right)\left(\gamma^{\mu} \underline{\Psi}_{ \pm}, \underline{\Omega}_{ \pm}\right) .
$$

Additionally, we find that the R-transformation does not change the reality condition (up to modifying $\alpha$ by a factor of $i$ ). For definiteness, we take the reality condition to be defined using $B_{-}$. First let us consider Weyl-compatible reality conditions. Given $\lambda^{i}$ with reality condition

$$
\left(\lambda_{ \pm}^{i}\right)^{*}=\alpha_{ \pm} B_{-} \lambda_{ \pm}^{j} L_{j i}
$$

we find

$$
\left(\Psi_{ \pm}^{i}\right)^{*}=-i \alpha_{ \pm} B_{-} \Psi_{ \pm}^{j} L_{j i}
$$

and we see the reality condition is unchanged, up to an overall phase.

Next we look at orthogonal Weyl-incompatible signatures, where we find that the reality condition is invariant.

$$
\left(\lambda_{ \pm}^{i}\right)^{*}=\alpha B_{-} \lambda_{\mp}^{j} L_{j i} \Longrightarrow\left(\Psi_{ \pm}^{i}\right)^{*}=\alpha B_{-} \Psi_{\mp}^{j} L_{j i}
$$

\section{E.1.2 Isotropic dimensions}

In isotropic dimensions, we work with Dirac spinors, called $\lambda^{i}$ and $\Psi^{i}$. For a reality condition of the form $\left(\lambda^{i}\right)^{*}=\alpha B \lambda^{j} L_{j i}$ we find, using (A.25) and (A.26):

$$
\left(\lambda^{i}\right)^{*}=\alpha B_{-} \lambda^{j} L_{j i} \Longrightarrow\left(\Psi^{i}\right)^{*}=(-1)^{t+1} i \alpha B_{+} \Psi^{j} L_{j i} .
$$

Since the overall phase can be absorbed in $\alpha$, the $R$-transformation can be used in isotropic dimensions to exchange $B_{-}$and $B_{+}$. In Weyl-incompatible signatures this is not a useful transformation, because $B_{ \pm}$have opposite $\epsilon$-type and the result is not one of our reality conditions. However in the Weyl-compatible case the $R$-transformation can be used to show that reality conditions are independent of the choice $B_{ \pm}$. For this to be an isomorphism of supersymmetry algebras, we also need that the bilinear form is invariant. Using that in isotropic dimensions $\gamma_{*} C_{ \pm}=C_{ \pm} \gamma_{*}$, we see that an isotropic vector-valued bilinear form is unchanged by this transformation

$$
\begin{aligned}
\left(\gamma^{\mu} \lambda^{i}\right)^{T} C_{ \pm} \chi^{j} M_{j i} & \rightarrow \frac{1}{2}\left(\gamma^{\mu}\left(1+i \gamma_{*}\right) \Psi^{i}\right)^{T} C_{ \pm}\left(1+i \gamma_{*}\right) \Omega^{j} M_{j i} \\
& =\left(\gamma^{\mu} \Psi^{i}\right)^{T} C_{ \pm} \Omega^{j} M_{j i}
\end{aligned}
$$

In section 7.4 the $R$-transformation is used (together with the $S$-transformation introduced in the next subsection) to show that isotropic Weyl-compatible supersymmetry algebras are classified by their R-symmetry group. 


\section{E.2 The S-transformations}

We define a family of invertible maps $S=S_{s}$ by

$$
S_{s}: \quad \lambda_{+}^{i} \rightarrow \lambda_{+}^{i}, \quad \lambda_{-}^{i} \rightarrow \lambda_{-}^{j} s_{j i}
$$

where $\left(s_{j i}\right)$ is an invertible matrix. In matrix notation this reads

$$
\left(\begin{array}{l}
\underline{\lambda}_{+} \\
\underline{\lambda}_{-}
\end{array}\right) \rightarrow S_{s}\left(\begin{array}{l}
\underline{\lambda}_{+} \\
\underline{\lambda}_{-}
\end{array}\right)=\left(\begin{array}{cc}
\mathbb{1} & 0 \\
0 & s^{T}
\end{array}\right)\left(\begin{array}{l}
\underline{\lambda}_{+} \\
\underline{\lambda}_{-}
\end{array}\right) .
$$

For further analysis we need to distinguish between orthogonal and isotropic dimensions.

\section{E.2.1 $S$-transformations and orthogonal bilinear forms}

In orthogonal dimensions the bilinear forms are entirely chiral, and the $S$-transformation leaves the vector-valued bilinear form $(C \otimes M)\left(\gamma^{\mu} \lambda_{+}, \chi_{+}\right)$on positive chirality spinors invariant by definition. On the negative chirality spinors $S$ acts as follows:

$$
\left(\gamma^{\mu} \lambda_{-}^{i}\right)^{T} C \chi_{-}^{j} M_{j i}=\left(\gamma^{\mu} \Psi_{-}^{k}\right)^{T} C \Omega_{-}^{l} M_{j i} s_{k i} s_{l j}
$$

To preserve $M$, that is $M_{j i} s_{k i} s_{l j}=M_{l k}$, the matrix $s$ needs to be orthogonal for $M=\delta$ and symplectic for $M=J$. This is the case in particular if $s \in\{\delta, I, J\}$ for $M=\delta$ and $s \in\{\delta, \tilde{I}, J\}$ for $M=J$. Note that these are the standard forms of $L$ which are used to impose reality conditions for a given $M \in\{\delta, J\}$ to select real forms of $\mathrm{O}(K, \mathbb{C})$ or $\operatorname{Sp}(K, \mathbb{C})$, respectively. ${ }^{32}$ Since in orthogonal dimensions $M$ is fixed by the dimension, it is useful to note that the $S$-map does not change the bilinear form on $\mathbb{C}^{K}$. It does, however, change the reality condition, see below for the case $s=L$ which is relevant for the classification of orthogonal Weyl-incompatible supersymmetry algebras.

\section{E.2.2 $S$-transformations and isotropic bilinear forms}

In the isotropic case the choice $s=J$ is the only one which preserves the standard form $M \in\{\delta, J\}$ for the bilinear form on $\mathbb{C}^{K}$. The map $s_{J}$ allows one to map $C_{ \pm} \otimes \delta$ to $C_{\mp} \otimes J$, where we choose the upper or lower sign depending on which choice leads to a super-admissible bilinear form.

Writing out the isotropic vector-valued bilinear form $\left(C_{ \pm} \otimes J\right)\left(\gamma^{\mu} \cdot, \cdot\right)$ explicitly gives:

$$
\left(\gamma^{\mu} \lambda_{+}^{i}\right) C_{ \pm} \chi_{-}^{j} J_{j i}+\left(\gamma^{\mu} \lambda_{-}^{i}\right)^{T} C_{ \pm} \chi_{+}^{j} J_{j i}
$$

Using (A.23) we can re-write this in terms of the other charge conjugation matrix

$$
\left(\gamma^{\mu} \lambda_{+}^{i}\right)^{T} C_{ \pm} \chi_{-}^{j} J_{j i}+\left(\gamma^{\mu} \lambda_{-}^{i}\right)^{T} C_{ \pm} \chi_{+}^{j} J_{j i}=-\left(\gamma^{\mu} \lambda_{+}^{i}\right)^{T} C_{\mp} \chi_{-}^{j} J_{j i}+\left(\gamma^{\mu} \lambda_{-}^{i}\right)^{T} C_{\mp} \chi_{+}^{j} J_{j i} .
$$

\footnotetext{
${ }^{32} L=I_{1,1}$ acts anti-isometrically for $M=J$. This case needs to be treated separately when constructing isomorphisms in the orthogonal Weyl-incompatible case, see appendix E.2.3. We don't need to use $S$ transformations for the orthogonal Weyl-compatible case.
} 
In our matrix notation, this equation is

$$
\left(\left(\gamma^{\mu} \lambda_{+}^{i}\right)^{T},\left(\gamma^{\mu} \lambda_{-}^{i}\right)^{T}\right) C_{ \pm}\left(\begin{array}{cc}
0 & J_{j i} \\
J_{j i} & 0
\end{array}\right)\left(\begin{array}{c}
\chi_{+}^{j} \\
\chi_{-}^{j}
\end{array}\right)=\left(\left(\gamma^{\mu} \lambda_{+}^{i}\right)^{T},\left(\gamma^{\mu} \lambda_{-}^{i}\right)^{T}\right) C_{\mp}\left(\begin{array}{cc}
0 & -J_{j i} \\
J_{j i} & 0
\end{array}\right)\left(\begin{array}{c}
\chi_{+}^{j} \\
\chi_{-}^{j}
\end{array}\right) .
$$

The other super-admissible bilinear form $\left(C_{\mp} \otimes \delta\right)\left(\gamma^{\mu} \cdot, \cdot\right)$ is

$$
\left(\gamma^{\mu} \Psi_{+}^{i}\right)^{T} C_{\mp} \Omega_{-}^{j} \delta_{i j}+\left(\gamma^{\mu} \Psi_{-}^{i}\right)^{T} C_{\mp} \Omega_{+}^{j} \delta_{i j}=\left(\left(\gamma^{\mu} \Psi_{+}^{i}\right)^{T},\left(\gamma^{\mu} \Psi_{-}^{i}\right)^{T}\right) C_{\mp}\left(\begin{array}{cc}
0 & \delta_{j i} \\
\delta_{j i} & 0
\end{array}\right)\left(\begin{array}{c}
\Omega_{+}^{j} \\
\Omega_{-}^{j}
\end{array}\right) .
$$

We look for a transformation of the form

$$
\left(\begin{array}{l}
\underline{\lambda}_{+} \\
\underline{\lambda}_{-}
\end{array}\right)=S\left(\begin{array}{l}
\underline{\Psi}_{+} \\
\underline{\Psi}_{-}
\end{array}\right)
$$

which maps (E.15) and (E.16) to on another. Then $S$ must satisfy

$$
S^{T}\left(\begin{array}{cc}
0 & J \\
-J & 0
\end{array}\right) S=\left(\begin{array}{ll}
0 & \mathbb{1} \\
\mathbb{1} & 0
\end{array}\right)
$$

which is solved by

$$
S=\left(\begin{array}{cc}
\mathbb{1} & 0 \\
0 & -J
\end{array}\right)
$$

Note that this map is an isomorphism between the complex supersymmetry algebras based on the bilinear forms $C_{ \pm} \otimes J$ and $C_{\mp} \otimes \delta$ in isotropic signatures. When using it for real supersymmetry algebras we need to take into account how it acts on reality conditions. This requires us to distinguish between Weyl-compatible and Weyl-incompatible signatures, and is done below.

\section{E.2.3 $S_{L}$ transformations and Weyl-incompatible reality conditions}

Consider the Weyl-incompatible reality condition

$$
\left(\lambda_{ \pm}^{i}\right)^{*}=\alpha B \lambda_{\mp}^{j} L_{j i}
$$

We can apply an $S_{s}$-transformation with $s=L^{-1}$ to change the reality condition. Given that

$$
\Psi_{+}^{i}=\lambda_{+}^{i}, \quad \Psi_{-}^{i}=\lambda_{-}^{j} L_{j i}^{-1}
$$

it is easy to see that

$$
\begin{aligned}
& \left(\Psi_{+}^{i}\right)^{*}=\left(\lambda_{+}^{i}\right)^{*}=\alpha B \lambda_{-}^{j} L_{j i}=\alpha B \Psi_{-}^{i} \\
& \left(\Psi_{-}^{i}\right)^{*}=\left(\lambda_{-}^{j}\right)^{*} L_{j i}^{-1}=\alpha B \lambda_{+}^{k} L_{k j} L_{j i}^{-1}=\alpha B \Psi_{+}^{i}
\end{aligned}
$$


Thus we can map a reality condition defined by any $L$ to a reality condition with $L=\delta$, and by using the inverse transformations we can map any choice of $L$ to any other choice $L^{\prime}$. In order to have isomorphic supersymmetry algebras, we need that the superbracket is invariant. As mentioned above, this is the case if $L$ is one of the canonical choices for the given $M$ listed in section 4.2, except for the combination $M=J, L=I_{1,1}$ where $L^{T} M L=-M$. In this case we use $s_{i I_{1,1}}$ which preserves the superbracket and modifies the reality condition by an irrelevant overall phase factor. In section $7.3 S$-transformations are used to show that for orthogonal, Weyl-incompatible signatures supersymmetry algebras are classified by their R-symmetry group.

\section{E.2.4 $S_{J}$ transformations in the isotropic Weyl-compatible signatures}

Consider spinors $\lambda_{ \pm}^{i}$ that obey a generic Weyl-compatible reality condition

$$
\left(\lambda_{ \pm}^{i}\right)^{*}=\alpha B_{( \pm)} \lambda_{ \pm}^{j} L_{j i}
$$

where the choice of $B_{( \pm)}$is independent of the chirality of the spinor. Under $S_{J} \lambda^{i}$ is related to

$$
\Psi_{+}^{i}=\lambda_{+}^{i}, \quad \Psi_{-}^{i}=-\lambda_{-}^{j} J_{j i} .
$$

Obviously $\Psi_{+}^{i}$ obeys the same reality condition as $\lambda_{+}^{i}$

$$
\left(\Psi_{+}^{i}\right)^{*}=\alpha B_{( \pm)} \Psi_{+}^{j} L_{j i}
$$

Calculating the reality condition for $\Psi_{-}^{i}$ is not so trivial:

$$
\begin{aligned}
\left(\Psi_{-}^{i}\right)^{*} & =-\left(\alpha B_{( \pm)} \lambda_{-}^{k} L_{k j}\right) J_{j i} \\
& =-\alpha B_{( \pm)} \Psi_{-}^{l} J_{l k} L_{k j} J_{j i} .
\end{aligned}
$$

For the two choices $L \in\{\delta, J\}$ we find that $\lambda_{ \pm}^{i}$ and $\Psi_{ \pm}^{i}$ obey the same reality condition:

$$
J_{l k} L_{k j} J_{j i}= \begin{cases}-\delta_{l i}, & L_{i j}=\delta_{i j}, \\ -J_{l i}, & L_{i j}=J_{i j} .\end{cases}
$$

This shows that $S_{J}$ exchanges the two bilinear forms $C \otimes \delta$ and $C^{\prime} \otimes J$, while preserving reality conditions based on $L=\delta$ and $L=J$. This is used in section 7.4 to show that isotropic Weyl-compatible supersymmetry algebras are classified by their R-symmetry group. To complete the argument, one also needs the $T$-transformation introduced in the next section.

\section{E.3 The T-transformation}

This transformation is only used in isotropic, Weyl compatible signatures, where it maps certain choices of $L$ to one another, while preserving the bilinear form. There are two cases. In the first case the bilinear form is $C \otimes \delta$ and the reality condition is

$$
\left(\lambda_{ \pm}^{i}\right)^{*}=\alpha B \lambda_{ \pm}^{j}\left(I_{p, q}\right)_{j i}
$$


In matrix notation this is

$$
\left(\begin{array}{l}
\underline{\lambda}_{+} \\
\underline{\lambda}_{-}
\end{array}\right)^{*}=\alpha B\left(\begin{array}{cc}
I_{p, q} & 0 \\
0 & I_{p, q}
\end{array}\right)\left(\begin{array}{l}
\underline{\lambda}_{+} \\
\underline{\lambda}_{-}
\end{array}\right) .
$$

One can show that if

$$
\left(\begin{array}{l}
\underline{\Psi}_{+} \\
\underline{\Psi}_{-}
\end{array}\right)=\left(\begin{array}{cccc}
\mathbb{1}_{p} & 0 & 0 & 0 \\
0 & i \mathbb{1}_{q} & 0 & 0 \\
0 & 0 & \mathbb{1}_{p} & 0 \\
0 & 0 & 0 & -i \mathbb{1}_{q}
\end{array}\right)\left(\begin{array}{l}
\underline{\lambda}_{+} \\
\underline{\lambda}_{-}
\end{array}\right)
$$

then $\Psi_{ \pm}$obey the reality condition

$$
\left(\begin{array}{l}
\underline{\Psi}_{+} \\
\underline{\Psi}_{-}
\end{array}\right)^{*}=\alpha B\left(\begin{array}{l}
\underline{\Psi}_{+} \\
\underline{\Psi}_{-}
\end{array}\right)
$$

where $L=I_{p, q}$ has been replaced by $L=\delta$. We remark that this transformation is not particularly useful in orthogonal dimensions, because it changes the bilinear form, and the result takes a non-standard form. However we only need to use $T$ in isotropic dimensions, where these reality conditions are paired with the bilinear form $C \otimes \delta$ which is unchanged:

$$
[C \otimes \delta](\underline{\lambda}, \underline{\chi})=[C \otimes \delta](\underline{\Psi}, \underline{\Omega})
$$

In the second case the bilinear form is $C^{\prime} \otimes J$, and the reality conditions involve $L=\tilde{I}_{2 r, 2 s}$ and $L=\delta$. These can be mapped using the $T$-transformation

$$
T=\left(\begin{array}{cccccccc}
\mathbb{1}_{r} & 0 & 0 & 0 & 0 & 0 & 0 & 0 \\
0 & i \mathbb{1}_{s} & 0 & 0 & 0 & 0 & 0 & 0 \\
0 & 0 & \mathbb{1}_{r} & 0 & 0 & 0 & 0 & 0 \\
0 & 0 & 0 & i \mathbb{1}_{s} & 0 & 0 & 0 & 0 \\
0 & 0 & 0 & 0 & \mathbb{1}_{r} & 0 & 0 & 0 \\
0 & 0 & 0 & 0 & 0 & -i \mathbb{1}_{s} & 0 & 0 \\
0 & 0 & 0 & 0 & 0 & 0 & \mathbb{1}_{r} & 0 \\
0 & 0 & 0 & 0 & 0 & 0 & 0 & -i \mathbb{1}_{s}
\end{array}\right),
$$

which leaves invariant the bilinear form $C^{\prime} \otimes J$. Together with the $S_{J}$-transformation, the $T$-transformation allows to show that isotropic Weyl-compatible supersymmetry algebras are classified by their R-symmetry group, see section 7.4.

\section{E.4 Relations between isotropic Weyl-incompatible supersymmetry algebras}

We start with a generic Weyl-incompatible reality condition

$$
\left(\lambda_{ \pm}^{i}\right)^{*}=\alpha B_{( \pm)} \lambda_{\mp}^{j} L_{j i}
$$

In Weyl-incompatible signatures $B_{ \pm}$have opposite $\epsilon$-type, and therefore the choice of $B$ is not free, but fixed by $L$. Here $B_{( \pm)}$refers to the choice which satisfies $B^{*} B=L^{2}$ and therefore in combination with $L$ defines a real structure. $B_{(\mp)}$ refers to the other $B$-matrix. 


\section{E.4.1 $S_{J}$-transformation: $(\delta, \delta) \rightarrow(J, J)$ and $(\delta, J) \rightarrow(J, \delta)$}

Under the $S_{J}$-transformation the transformed spinors, $\Psi_{ \pm}^{i}$ obey

$$
\begin{aligned}
& \left(\Psi_{+}^{i}\right)^{*}=\left(\lambda_{+}^{i}\right)^{*}=\alpha B_{( \pm)} \lambda_{-}^{j} L_{j i}=\alpha B_{( \pm)} \Psi_{-}^{k} J_{k j} L_{j i}=-\alpha B_{(\mp)} \Psi_{-}^{k} J_{k j} L_{j i}, \\
& \left(\Psi_{-}^{i}\right)^{*}=-\left(\lambda_{-}^{i}\right)^{*} J_{j i}=-\alpha B_{( \pm)} \lambda_{+}^{j} L_{k j} J_{j i}=-\alpha B_{( \pm)} \Psi_{+}^{k} L_{k j} J_{j i}=-\alpha B_{(\mp)} \Psi_{+}^{k} L_{k j} J_{j i},
\end{aligned}
$$

where in the last step we used (A.25) to re-write the reality condition in terms of the other $B$-matrix. This is needed because $L=\delta$ and $L=J$ have opposite $\epsilon$-type. Using that

$$
\begin{aligned}
J_{k j} \delta_{j i} & =J_{k i}, & \delta_{k j} J_{j i} & =J_{k i}, \\
J_{k j} J_{j i} & =-\delta_{k i}, & & J_{k j} J_{j i}=-\delta_{k i}
\end{aligned}
$$

we find

$$
\begin{aligned}
& \left(\lambda^{i}\right)^{*}=\alpha B_{( \pm)} \lambda^{i} \Rightarrow\left(\Psi^{i}\right)^{*}=-\alpha B_{(\mp)} \Psi^{j} J_{j i} \\
& \left(\lambda^{i}\right)^{*}=\alpha B_{( \pm)} \lambda^{j} J_{j i} \Rightarrow\left(\Psi^{i}\right)^{*}=\alpha B_{(\mp)} \Psi^{i} .
\end{aligned}
$$

This shows that the algebras defined by the data $(M, L)=(\delta, \delta)$ and $(J, J)$, are related by the $s_{J}$ transformation. These are in fact the standard Majorana and the symplectic Majorana condition, both with R-symmetry group $\mathrm{U}(K)$. This shows that the two ways of realizing this R-symmetry group lead to isomorphic supersymmetry algebras, see section 7.5 and the diagram in (7.1). For the same reasons, the pairs $(M, L)=(\delta, J)$ and $(J, \delta)$ which have R-symmetry group $\mathrm{U}(k, k)$ are also related to one another by $S_{J}$. There are two other ways of obtaining a $\mathrm{U}(k, k)$ R-symmetry group: with $(M, L)=\left(\delta, I_{k, k}\right)$ and $\left(J, \tilde{I}_{2 r, 2 s}\right)$, which will now be discussed.

\section{E.4.2 F-transformation: $(\delta, J) \rightarrow\left(\delta, I_{k, k}\right)$}

We start with an algebra with $(M, L)=(\delta, J)$, so the initial spinors have reality condition

$$
\left(\lambda_{ \pm}^{i}\right)^{*}=\alpha B_{( \pm)} \lambda_{\mp}^{j} J_{j i} .
$$

To transform this to an algebra with $(M, L)=\left(\delta, I_{k, k}\right)$, we apply the transformation (using the 'doubled again' matrix notation) $\Psi^{I}=\lambda^{J} F_{J I}$ where

$$
F=\frac{1+i}{2}\left(\begin{array}{cccc}
-i \mathbb{1}_{k} & \mathbb{1}_{k} & 0 & 0 \\
\mathbb{1}_{k} & -i \mathbb{1}_{k} & 0 & 0 \\
0 & 0 & \mathbb{1}_{k} & -i \mathbb{1}_{k} \\
0 & 0 & -i \mathbb{1}_{k} & \mathbb{1}_{k}
\end{array}\right)
$$

The transformed spinors obey the reality condition

$$
\left(\Psi^{i}\right)^{*}=i \alpha B_{(\mp)} \Psi^{j}\left(I_{k, k}\right)_{j i}
$$

Since $J$ and $I_{k, k}$ have opposite $\epsilon$-type we have used (A.25) to change the $B$-matrix accordingly. 


\section{E.4.3 $G$-transformation: $\left(J, \tilde{I}_{2 r, 2 s}\right) \rightarrow(J, \delta)$}

Let $\lambda^{i}$ be the spinors from a supersymmetry algebra with $(M, L)=\left(J, \tilde{I}_{2 r, 2 s}\right)$ with reality condition

$$
\left(\lambda^{i}\right)^{*}=\alpha B_{( \pm)} \lambda^{j}\left(\tilde{I}_{2 r, 2 s}\right)_{j i} .
$$

We define $\Psi^{i}=\lambda^{j} G_{j i}$ where

$$
G=\left(\begin{array}{cccc}
\mathbb{1}_{r} & 0 & 0 & 0 \\
0 & i \mathbb{1}_{s} & 0 & 0 \\
0 & 0 & \mathbb{1}_{r} & 0 \\
0 & 0 & 0 & -i \mathbb{1}_{s}
\end{array}\right) .
$$

Note that this matrix has size $(2 r+2 s) \times(2 r+2 s)$, that is, we are not using the matrix notation.

One can show that

$$
\left(\Psi^{i}\right)^{*}=\alpha B_{( \pm)} \Psi^{i}
$$

so that $G$ maps a reality condition with $L=\tilde{I}_{2 r, 2 s}$ to a standard Majorana condition with $L=\delta$. Moreover, one can show that the vector-valued bilinear form $(C \otimes J)\left(\gamma^{\mu} \cdot, \cdot\right)$ is invariant. Therefore $G$ defines an isomorphism between isotropic Weyl-incompatible algebras with $(M, L)=\left(J, \tilde{I}_{2 r, 2 s}\right)$ and $(J, \delta)$, as indicated in the diagram (7.1) in section 7.5. This almost completes the classification of isotropic Weyl incompatible supersymmetry algebras, except for one special case that is not covered by the $G$-transformation.

\section{E.4.4 Remaining case $\left(J, I_{1,1}\right) \rightarrow(J, \delta)$}

The reality conditions $L=\tilde{I}_{2 r, 2 s}$ only cover R-symmetry groups $\mathrm{U}(k, k)=\mathrm{U}(r+s, r+s)$ with $k>1$, since $r, s \geq 1$. For $k=1$ we have a different canonical representative, namely $L=I_{1,1}$. However, this case was already covered in [17], where the isomorphisms between the isotropic, Weyl-incompatible supersymmetry algebras in signature $(1,3)$ with R-symmetry groups $\mathrm{U}(2)$ and $\mathrm{U}(1,1)$ have been worked out. If one performs the same computation in any other signature, the only detail which can change is which of the two matrices $B_{ \pm}$defines a real, and which defines a quaternionic structures. Since this does not change whether an isomorphism exists or not, the results of [17] imply that all isotropic Weyl incompatible supersymmetry algebras with R-symmetry group $\mathrm{U}(1,1)$ are isomorphic. ${ }^{33}$ This completes proving that isotropic, Weyl-incompatible supersymmetry algebra are classified by their Rsymmetry group, and as well all statements about classification of supersymmetry algebras made in section 7 .

\section{F Dimensional reduction}

In this section we derive the general formulas which allow one to perform spacelike and timelike dimensional reductions starting in an arbitrary signature.

\footnotetext{
${ }^{33}$ The relevant transformation is called $V$ in [17]. Note that there is a typo in the diagram which represents the isomorphisms. Also note that the off-diagonal matrix $\eta$ used in [17] is related to $I_{1,1}$ by an additional basis transformation.
} 


\section{F.1 Odd to even dimensions}

In this section we describe how the reduction from odd to even dimensions works in general. We use the following conventions: the space-time indices of the higher dimensional theory are $M=0, \ldots, D$. When performing a spacelike reduction we remove the final direction (when going from $(D+1)$ to $D$ dimensions this is the $D$-th direction) and when performing a timelike reduction we remove the 0 -th direction. Therefore the lower dimensional spacetime indices are $\mu=1, \ldots, D$ for a timelike reduction or $\mu=0, \ldots, D-1$ for a spacelike reduction.

When we reduce from an odd to an even space-time dimension, the dimension of the Dirac spinor module does not decrease, which makes reductions from odd to even dimensions simpler than the second case. We relate the higher-dimensional spinors and $\gamma$-matrices to the lower ones as follows:

$$
\lambda_{(D+1)}^{i}=\lambda_{(D)}^{i}, \quad \Gamma_{M}=\left\{\begin{array}{l}
\left\{\gamma_{\mu}, \gamma_{(D+1)}=\gamma_{*}\right\}, \quad \text { for spacelike reduction, } \\
\left\{\gamma_{0}=i \gamma_{*}, \gamma_{\mu}\right\}, \quad \text { for timelike reduction }
\end{array}\right.
$$

The 'extra' $\gamma$-matrix of the higher-dimensional theory is proportional to the chirality operator $\gamma_{*}$ of the lower-dimensional one. We choose representations such that for a spacelike reduction $\Gamma_{(D+1)}=\gamma_{*}$ and for a timelike reduction $\Gamma_{0}=i \gamma_{*}$. This is always possible.

The charge conjugation matrix of the $(D+1)$-dimensional theory is equal to one of the two charge conjugation matrices in the even $D$ dimensions. From table 2 we can infer that under reduction it becomes $C_{+}$, if the lower-dimensional theory is orthogonal, and $C_{-}$if it is isotropic. The corresponding bilinear form of the reduced theory is $C_{+} \otimes M$ or $C_{-} \otimes M$ with the bilinear form $M$ inherited from the parent theory.

The reality condition is likewise inherited from the higher dimensional theory, though one needs to rewrite the $B$-matrix in terms of the lower dimensional $B$ matrices. When going from odd to even dimensions the dimensionally reduced $B$ matrices satisfy, for orthogonal dimensions

$$
\begin{aligned}
& B^{(t, s)}=\left(C\left(A^{(t, s)}\right)^{-1}\right)^{T}=\left(C_{-}\left(A^{(t, s-1)}\right)^{-1}\right)^{T}=B_{+}^{(t, s-1)}, \\
& B^{(t, s)}=(-1)^{t}\left(-i C_{-}\left(A^{(t-1, s)}\right)^{-1}\right)^{T}=(-1)^{t} B_{-}^{(t-1, s)},
\end{aligned}
$$

and for isotropic dimensions

$$
\begin{aligned}
& B^{(t, s)}=\left(C\left(A^{(t, s)}\right)^{-1}\right)^{T}=\left(C_{-}\left(A^{(t, s-1)}\right)^{-1}\right)^{T}=B_{-}^{(t, s-1)}, \\
& B^{(t, s)}=(-1)^{t}\left(-i C_{+}\left(A^{(t-1, s)}\right)^{-1}\right)^{T}=(-1)^{t+1} i B_{+}^{(t-1, s)} .
\end{aligned}
$$

At least one of the $\epsilon$-quaternionic structures in the reduced signature has the same $\epsilon$ as the $\epsilon$-quaternionic structure in the parent signature when going from odd dimensions to even dimensions, so that the daughter theories can have the same $L$. More details which allow to derive the above relations can be found in [17].

\section{F.2 Even to odd dimensions}

This case is a bit more complicated since the dimensionality of Dirac spinors halves as we dimensionally reduce. Morally speaking we can equate the Weyl spinors of the parent 
theory with the Dirac spinors of the daughter theory. The charge conjugation matrices and $\gamma$-matrices reduce in size, too. Relating the two theories requires some care, though fortunately there are not too many possibilities.

In the odd-dimensional daughter signature, we only have a single $C$, but it will always be related to the $C$-matrix of one of its two even-dimensional parents. By inspection of our tables, $C$ has the same invariants as $C_{-}$if the parent theory is orthogonal, while it has the same invariants as $C_{+}$if the parent theory is isotropic. This explains why we can embed $C$ into the higher dimensional $C_{ \pm}$by

$$
C_{-}^{(D+1)}=C^{(D)} \otimes \sigma_{1}, \quad \mathrm{D}=5,9 \quad \text { or } \quad C_{+}^{(D+1)}=C^{(D)} \otimes 1, \quad \mathrm{D}=3,7,11 .
$$

The bilinear form on the extended spinor module of the parent theory is $C^{(D+1)} \otimes M$ with whatever $C^{(D+1)}$ is in the above formula and where $M=\{\delta, J\}$ is the correct choice to make the bilinear form super-admissible. If the standard bilinear form in the parent theory is different from what is obtained this way, we can use the maps constructed in appendix $\mathrm{E}$ to bring the bilinear form to its canonical form.

Finally we have to choose an embedding of the $\gamma$-matrices. Those in the parent theory will be called $\Gamma_{M}$, with $M=1, \ldots, D+1$ if we are reducing along a spacelike direction and $M=0, \ldots, D$ if we are reducing along a timelike direction. The $\gamma$-matrices of the daughter theory are $\gamma_{\mu}$, with $\mu=1, \ldots, D$ always. We embed the $\gamma$-matrices as follows:

$$
\Gamma_{\mu}=\gamma_{\mu} \otimes \sigma_{1}, \quad \Gamma_{(D+1)}=1 \otimes \sigma_{2} \quad \text { or } \quad \Gamma_{0}=i 1 \otimes \sigma_{2} .
$$

We remove either $\Gamma_{(D+1)}$ or $\Gamma_{0}$ depending on whether we reduce along a spacelike or timelike direction. We define $\Gamma_{*}$ according to the conventional

$$
\Gamma_{*}=(-i)^{D / 2+t} \prod_{\mu} \Gamma_{\mu}
$$

and choose the $\gamma_{\mu}$ such that

$$
\Gamma_{*}=1 \otimes \sigma_{3} .
$$

Note that it is always possible, as the daughter theory is in odd dimensions. Therefore there are two inequivalent representations of the Clifford algebra that are distinguished by the sign of $\gamma_{(D)}$. We can therefore choose the representation such that the above relation holds.

For completeness we then have the other charge conjugation matrix, from (A.23) given as

$$
C_{+}^{(D+1)}=C^{(D)} \otimes \sigma_{2} \quad \mathrm{D}=5,9 \quad \text { or } \quad C_{-}^{(D+1)}=C^{(D)} \otimes \sigma_{3} \quad \mathrm{D}=3,7,11 .
$$

We can therefore decompose the $D+1$ dimensional spinors into $D$-dimensional spinors according to

$$
\lambda_{+}^{i}=\psi^{i} \otimes\left(\begin{array}{l}
1 \\
0
\end{array}\right), \quad \lambda_{-}^{\hat{i}}=\psi^{i+K_{+}} \otimes\left(\begin{array}{l}
0 \\
1
\end{array}\right)
$$

where $\lambda_{+}^{i}$ and $\lambda_{-}^{\hat{i}}$ are the spinors in $D+1$ dimensions, of which we have $K_{+}$and $K_{-}$ respectively, and $\psi^{i}$ the spinors in $d$ dimensions, of which we now have $K_{+}+K_{-}$. We 
may need to transform the $\psi^{i}$ quantities to put the bilinear form and reality condition into canonical forms.

We are now able to dimensionally reduce the vector-valued bilinear form. We have two cases, namely orthogonal and isotropic vector-valued bilinear forms. We begin with an orthogonal vector-valued bilinear form with $K_{+}$positive and $K_{-}$negative chirality spinors

$$
\begin{aligned}
& \left(\Gamma^{M} \lambda_{+}^{i}\right)^{T} C^{(D+1)} \chi_{+}^{j} M_{j i}+\left(\Gamma^{M} \lambda_{-}^{\hat{i}}\right)^{T} C^{(D+1)} \chi_{-}^{\hat{j}} M_{\hat{j} \hat{i}}^{\prime} \\
& =\left(\gamma^{\mu} \psi^{i}\right)^{T} C^{(D)} \phi^{j} M_{j i} \otimes\left(\sigma_{1}\left(\begin{array}{l}
1 \\
0
\end{array}\right)\right)^{T} \sigma_{1}\left(\begin{array}{l}
1 \\
0
\end{array}\right)+\left(\gamma^{\mu} \psi^{\tilde{i}}\right)^{T} C^{(D)} \phi^{\tilde{j}} M_{\tilde{j} \tilde{i}}^{\prime} \otimes\left(\sigma_{1}\left(\begin{array}{l}
0 \\
1
\end{array}\right)\right)^{T} \sigma_{1}\left(\begin{array}{l}
0 \\
1
\end{array}\right) \\
& =\left(\left(\gamma^{\mu} \psi^{i}\right)^{T} C^{(D)} \phi^{j} M_{j i}+\left(\gamma^{\mu} \psi^{\tilde{i}}\right)^{T} C^{(D)} \phi^{\tilde{j}} M_{\tilde{j} \tilde{j}}^{\prime}\right) \otimes 1 \\
& =\left(\gamma^{\mu} \psi^{i}\right) C^{(D)} \phi^{j}\left(\begin{array}{cc}
M & 0 \\
0 & M^{\prime}
\end{array}\right)_{j i} \otimes 1 .
\end{aligned}
$$

Here $i, j=1, \ldots, K$ and $\tilde{i}, \tilde{j}=K_{+}+1, \ldots, K_{+}+K_{-}$until the final line where we have combined the indices so that $i, j=1, \ldots, K_{+}+K_{-} . M$ and $M^{\prime}$ will be of the same form, either $\delta$ or $J$, but are $K_{+} \times K_{+}$and $K_{-} \times K_{-}$matrices in the reduced theory, respectively.

Note if $M=\delta$ this is already correctly lined up so that the $D$-dimensional theory has the vector-valued bilinear form

$$
\left(\gamma^{\mu} \psi^{i}\right) C^{(d)} \phi^{j} \delta_{j i}, \quad i=1, \ldots, K_{+}+K_{-} .
$$

However, if $M=J$ we are not in the canonical form, in that

$$
\left(\begin{array}{cc}
J_{K_{+}} & 0 \\
0 & J_{K_{-}}
\end{array}\right) \neq J_{K_{+}+K_{-}} .
$$

We then need a change of basis for $\psi^{i}$ to realign the spinors into a canonical form (this will also affect the reality condition).

For isotropic dimensions, where $K_{+}=K_{-}$and $M=M^{\prime}$, we find the following

$$
\begin{aligned}
& \left(\Gamma^{M} \lambda_{+}^{i}\right)^{T} C^{(D+1)} \chi_{-}^{j} M_{j i}+\left(\Gamma^{M} \lambda_{-}^{i}\right)^{T} C^{(D+1)} \chi_{+}^{j} M_{j i} \\
& =\left(\gamma^{\mu} \psi^{i}\right)^{T} C^{(D)} \phi^{\tilde{j}} M_{\tilde{j} i} \otimes\left(\sigma_{1}\left(\begin{array}{l}
1 \\
0
\end{array}\right)\right)^{T}\left(\begin{array}{l}
1 \\
0
\end{array}\right)+\left(\gamma^{\mu} \psi^{\tilde{i}}\right)^{T} C^{(D)} \phi^{j} M_{j \tilde{i}} \otimes\left(\sigma_{1}\left(\begin{array}{l}
0 \\
1
\end{array}\right)\right)^{T}\left(\begin{array}{l}
0 \\
1
\end{array}\right) \\
& =\left(\gamma^{\mu} \psi^{i}\right)^{T} C^{(D)} \phi^{j}\left(\begin{array}{cc}
0 & M \\
M & 0
\end{array}\right) \otimes 1 .
\end{aligned}
$$

Here $i, j=1, \ldots, K$ and $\tilde{i}, \tilde{j}=K+1, \ldots, 2 K$ until the final line where we have combined the indices so that $i, j=1, \ldots, 2 K$. In the final expression $M$ represent the original $K \times K$ Gram matrices inherited from the parent theory. We will then need a basis transformation to obtain the canonical form.

Next we consider the reduction of reality conditions. Due to different embeddings of $C$ into the parent theory, we have different factorisations of the $B$ matrices depending on 
whether the parent is orthogonal or isotropic. For a spacelike dimension from $(t, s+1)$ to $(t, s)$ we find

$$
\begin{aligned}
& B_{+}^{(t, s+1)}= \begin{cases}-B^{(t, s)} \otimes \sigma_{1}^{t} \sigma_{2} & \text { for orthogonal parent, } \\
B^{(t, s)} \otimes \sigma_{1}^{t} & \text { for isotropic parent, }\end{cases} \\
& B_{-}^{(t, s+1)}= \begin{cases}B^{(t, s)} \otimes \sigma_{1}^{t+1} & \text { for orthogonal parent, } \\
B^{(t, s)} \otimes \sigma_{1}^{t} \sigma_{3} & \text { for isotropic parent. }\end{cases}
\end{aligned}
$$

Along a timelike direction, from $(t+1, s)$ to $(t, s)$ we find

$$
\begin{aligned}
& B_{+}^{(t+1, s)}= \begin{cases}(-1)^{t+1} i B^{(t, s)} \otimes \sigma_{1}^{t} & \text { for orthogonal parent, } \\
i B^{(t, s)} \otimes \sigma_{2} \sigma_{1}^{t} & \text { for isotropic parent, }\end{cases} \\
& B_{-}^{(t+1, s)}= \begin{cases}i B^{(t, s)} \otimes \sigma_{2} \sigma_{1}^{t+1} & \text { for orthogonal parent } \\
(-1)^{t+1} B^{(t, s)} \otimes \sigma_{1}^{t+1} & \text { for isotropic parent. }\end{cases}
\end{aligned}
$$

Note the presence of powers of $\sigma^{1}$ resulting from the decomposition of the matrix $A$, which is the product of all timelike $\gamma$-matrices.

Open Access. This article is distributed under the terms of the Creative Commons Attribution License (CC-BY 4.0), which permits any use, distribution and reproduction in any medium, provided the original author(s) and source are credited.

\section{References}

[1] C.M. Hull, Timelike T duality, de Sitter space, large $N$ gauge theories and topological field theory, JHEP 07 (1998) 021 [hep-th/9806146] [INSPIRE].

[2] C.M. Hull, Duality and the signature of space-time, JHEP 11 (1998) 017 [hep-th/9807127] [INSPIRE].

[3] R. Dijkgraaf, B. Heidenreich, P. Jefferson and C. Vafa, Negative Branes, Supergroups and the Signature of Spacetime, JHEP 02 (2018) 050 [arXiv: 1603.05665] [INSPIRE].

[4] R. Blumenhagen, M. Brinkmann, A. Makridou, L. Schlechter and M. Traube, dS Spaces and Brane Worlds in Exotic String Theories, JHEP 06 (2020) 077 [arXiv:2002.11746] [INSPIRE].

[5] S. Ferrara, Spinors, superalgebras and the signature of space-time, in 9th Marcel Grossmann Meeting on Recent Developments in Theoretical and Experimental General Relativity, Gravitation and Relativistic Field Theories (MG 9), pp. 68-83 (2001) [DOI] [hep-th/0101123] [INSPIRE].

[6] E. Cremmer, I.V. Lavrinenko, H. Lü, C.N. Pope, K.S. Stelle and T.A. Tran, Euclidean signature supergravities, dualities and instantons, Nucl. Phys. B 534 (1998) 40 [hep-th/9803259] [INSPIRE].

[7] C.M. Hull and B. Julia, Duality and moduli spaces for timelike reductions, Nucl. Phys. B 534 (1998) 250 [hep-th/9803239] [INSPIRE].

[8] J.B. Gutowski and W.A. Sabra, Euclidean $N=2$ Supergravity, Phys. Lett. B 718 (2012) 610 [arXiv: 1209.2029] [INSPIRE]. 
[9] W.A. Sabra and O. Vaughan, Euclidean Supergravity in Five Dimensions, Phys. Lett. B $\mathbf{7 6 0}$ (2016) 14 [arXiv: 1603.09244] [INSPIRE].

[10] W. Sabra and O. Vaughan, 10D to 4D Euclidean Supergravity over a Calabi-Yau three-fold, Class. Quant. Grav. 33 (2016) 015010 [arXiv: 1503.05095] [InSPIRE].

[11] W.A. Sabra, Special geometry and space-time signature, Phys. Lett. B 773 (2017) 191 [arXiv: 1706.05162] [INSPIRE].

[12] V. Cortés, C. Mayer, T. Mohaupt and F. Saueressig, Special geometry of Euclidean supersymmetry. I: Vector multiplets, JHEP 03 (2004) 028 [hep-th/0312001] [INSPIRE].

[13] V. Cortés, C. Mayer, T. Mohaupt and F. Saueressig, Special geometry of euclidean supersymmetry. II: Hypermultiplets and the c-map, JHEP 06 (2005) 025 [hep-th/0503094] [INSPIRE].

[14] V. Cortés and T. Mohaupt, Special Geometry of Euclidean Supersymmetry III: The Local r-map, instantons and black holes, JHEP 07 (2009) 066 [arXiv:0905.2844] [INSPIRE].

[15] V. Cortés, P. Dempster, T. Mohaupt and O. Vaughan, Special Geometry of Euclidean Supersymmetry IV: the local c-map, JHEP 10 (2015) 066 [arXiv:1507.04620] [INSPIRE].

[16] L. Gall and T. Mohaupt, Five-dimensional vector multiplets in arbitrary signature, JHEP 09 (2018) 053 [arXiv: 1805.06312] [INSPIRE].

[17] V. Cortés, L. Gall and T. Mohaupt, Four-dimensional vector multiplets in arbitrary signature (I), Int. J. Geom. Meth. Mod. Phys. 17 (2020) 2050150 [arXiv: 1907.12067] [InSPIRE].

[18] D. Klemm and M. Nozawa, Geometry of Killing spinors in neutral signature, Class. Quant. Grav. 32 (2015) 185012 [arXiv: 1504.02710] [INSPIRE].

[19] J.B. Gutowski and W.A. Sabra, Real Killing Spinors in Neutral Signature, JHEP 11 (2019) 173 [arXiv: 1905.01910] [INSPIRE].

[20] W.A. Sabra, Kasner Branes with Arbitrary Signature, Phys. Lett. B 809 (2020) 135694 [arXiv: 2005.03953] [INSPIRE].

[21] W.A. Sabra, Hypersymplectic geometry and supersymmetric solutions in $(t, s) 5 D$ supergravity, Phys. Rev. D 104 (2021) 046012 [arXiv:2105.00806] [INSPIRE].

[22] D.V. Alekseevsky and V. Cortés, Classification of N-(super)-extended Poincare Algebras and bilinear invariants of spinor representations of $\operatorname{Spin}(p, q)$, Commun. Math. Phys. 183 (1997) 477 [math/9511215].

[23] H.B. Lawson and M.-L. Michelsohn, Spin Geometry, Princeton University Press (1989).

[24] D.V. Alekseevsky, V. Cortés, C. Devchand and A. Van Proeyen, Polyvector superPoincaré algebras, Commun. Math. Phys. 253 (2004) 385 [hep-th/0311107] [INSPIRE].

[25] A. Van Proeyen, Tools for supersymmetry, Ann. U. Craiova Phys. 9 (1999) 1 [hep-th/9910030] [INSPIRE].

[26] S. Lang, Algebra, Springer (2002).

[27] R. Gilmore, Lie Groups, Lie Algebras and Some of Their Applications, Wiley Interscience Publication (1974).

[28] S. Cecotti, S. Ferrara and L. Girardello, Geometry of Type II Superstrings and the Moduli of Superconformal Field Theories, Int. J. Mod. Phys. A 4 (1989) 2475 [InSPIRE]. 
[29] S. Ferrara and S. Sabharwal, Quaternionic Manifolds for Type II Superstring Vacua of Calabi-Yau Spaces, Nucl. Phys. B 332 (1990) 317 [inSPIRE].

[30] M. Bodner, A.C. Cadavid and S. Ferrara, $(2,2)$ vacuum configurations for type IIA superstrings: $N=2$ supergravity Lagrangians and algebraic geometry, Class. Quant. Grav. 8 (1991) 789 [INSPIRE].

[31] G.L. Cardoso and T. Mohaupt, Special geometry, Hessian structures and applications, Phys. Rept. 855 (2020) 1 [arXiv: 1909.06240] [InSPIRE].

[32] M. Médevielle, T. Mohaupt and G. Pope, Type-II Calabi-Yau compactifications, T-duality and special geometry in general spacetime signature, to appear.

[33] J. Gutowski, T. Mohaupt and G. Pope, Cosmological Solutions, a New Wick-Rotation, and the First Law of Thermodynamics, JHEP 03 (2021) 293 [arXiv:2008.06929] [INSPIRE].

[34] J. Gutowski, T. Mohaupt and G. Pope, From static to cosmological solutions of $\mathcal{N}=2$ supergravity, JHEP 08 (2019) 172 [arXiv: 1905.09167] [INSPIRE].

[35] G.W. Moore, Finite in all directions, hep-th/9305139 [INSPIRE].

[36] E. Bergshoeff and A. Van Proeyen, The Many faces of OSp(1|32), Class. Quant. Grav. 17 (2000) 3277 [hep-th/0003261] [INSPIRE].

[37] E.A. Bergshoeff, J. Hartong, A. Ploegh, J. Rosseel and D. Van den Bleeken, Pseudo-supersymmetry and a tale of alternate realities, JHEP 07 (2007) 067 [arXiv: 0704.3559] [INSPIRE].

[38] O. Hohm and H. Samtleben, The many facets of exceptional field theory, PoS CORFU2018 (2019) 098 [arXiv: 1905.08312] [INSPIRE].

[39] L. Gall, Supersymmetry Algebras in Arbitary Signature and Dimension, Ph.D. Thesis, Department of Mathematical Sciences, University of Liverpool (2019) [DOI].

[40] D.Z. Freedman and A. Van Proeyen, Supergravity, Cambridge University Press (2012) [INSPIRE]. 